Affecting drag in turbulent Taylor-Couette flow

Ruben Adriaan Verschoof 


\section{Graduation members:}

Prof. dr. ir. J.W.M. Hilgenkamp (chairman) University of Twente Prof. dr. ret. nat. D. Lohse (supervisor) University of Twente

Prof. dr. C. Sun (supervisor)

Dr. S. G. Huisman (co-supervisor)

Prof. dr. ir. C. H. Venner

Prof. dr. ir. R. G. H. Lammertink

Prof. dr. ir. T. J. C. van Terwisga

Prof. dr. G. H. McKinley
Tsinghua University, University of Twente

University of Twente

University of Twente

University of Twente

Delft University of Technology

Massachusetts Institute of Technology

\section{$\widehat{N W O} \mid \begin{aligned} & \text { Applied and } \\ & \text { Engineering Sciences }\end{aligned}$}

The work in this thesis was carried out at the Physics of Fluids group of the Faculty of Science and Technology of the University of Twente. This work is part of the research programme ShipDRAC with project number 13265, which is financed by the Netherlands Organisation for Scientific Research (NWO).

Dutch title:

Het beïnloeden van wrijving in turbulente Taylor-Couette stromingen

Publisher:

Ruben A. Verschoof, Physics of Fluids, University of Twente, P.O. Box 217, 7500 AE Enschede, The Netherlands

Copyright (c) Ruben A. Verschoof, Enschede, 2018. All rights reserved.

No part of this work may be reproduced or transmitted, in any form or by any means, electronic or mechanical, including photocopying and recording, or by any information storage or retrieval system, except as expressly permitted by the publisher.

Cover design:

Atmospheric and oceanic flow systems both deal with the topics studied in this thesis: transient effects, roughness, and multiphase flows. The motivation for the presented air lubrication studies stems from the maritime industry, in which air lubrication is seen as a highly promising method to reduce the friction and thus the fuel consumption. Photo by @builtbymath on unsplash.com.

ISBN: 978-90-365-4525-9

DOI: $10.3990 / 1.9789036545259$

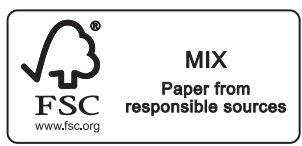




\title{
AFFECTING DRAG IN TURBULENT TAYLOR-COUETTE FLOW
}

\author{
DISSERTATION
}

to obtain

the degree of doctor at the University of Twente, on the authority of the rector magnificus,

Prof. dr. T.T.M. Palstra,

on account of the decision of the graduation committee,

to be publicly defended

on Friday the 1st of June 2018 at 16:45

by

Ruben Adriaan Verschoof

Born on the 7th of October 1990

in Ede, The Netherlands 
This dissertation has been approved by the supervisors:

Prof. dr. ret. nat. D. Lohse

Prof. dr. C. Sun

and the co-supervisor:

Dr. S.G. Huisman 


\section{Contents}

Introduction 1

\begin{tabular}{ll}
\hline I Transient turbulence & 7
\end{tabular}

\begin{tabular}{|lll|}
\hline 1 & Self-similar decay of high Reynolds number Taylor-Couette turbu- & \\
\hline \hline & lence & 9
\end{tabular}

1.1 Introduction . . . . . . . . . . . . . . . . . . . . . . . . . . . . . . . . . . . .

1.2 Experiments . . . . . . . . . . . . . . . . . . . . 11

1.3 Results and analysis . . . . . . . . . . . . . . . . . . . . . . 12

1.4 Conclusions . . . . . . . . . . . . . . . . . . . 17

2 Periodically driven Taylor-Couette turbulence 19

2.1 Introduction . . . . . . . . . . . . . . . . . 20

2.2 Method . . . . . . . . . . . . . . . . . . . . . . . 21

2.3 Results and analysis . . . . . . . . . . . . . . . . . . . . . . . . . . . . . . . . 5

2.4 Summary and conclusions . . . . . . . . . . . . . . . . . . . 30

II Roughness in turbulence $\quad 31$

3 Wall roughness induces asymptotic ultimate turbulence 33

3.1 Introduction . . . . . . . . . . . . . . . . 34

3.2 Global scaling relations . . . . . . . . . . . . . . . . . . . . 37

3.3 Local flow organization and profiles . . . . . . . . . . . . . . . . . . . 41

3.4 Controlling ultimate turbulence . . . . . . . . . . . . . . . . . . . . . . . . . . . . . . . . . . . . . . . . .

3.5 Methods . . . . . . . . . . . . . . . . . . . . 43

4 Rough wall turbulent Taylor-Couette flow: the effect of the rib $\begin{array}{ll}\text { height } & 49\end{array}$

4.1 Introduction . . . . . . . . . . . . . . . . . . . . . . 50

4.2 Methods . . . . . . . . . . . . . . . . . . . . 52

4.3 Global response: torque and its scaling . . . . . . . . . . . . . . . . 54 


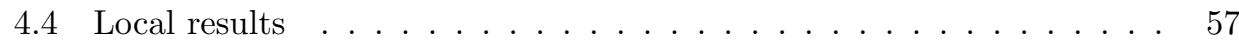

$4.5 \quad$ Optimal transport $\ldots \ldots \ldots \ldots \ldots \ldots$

4.6 Conclusions and Outlook $\ldots \ldots \ldots \ldots \ldots$

III Air lubrication in turbulent flows 63

\begin{tabular}{|lll}
5 & Bubble drag reduction requires large bubbles & 65
\end{tabular}

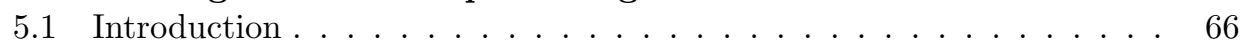

5.2 Experiments . . . . . . . . . . . . . . . . . . . . 66

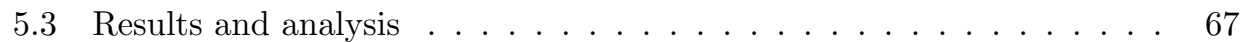

5.4 Conclusions . . . . . . . . . . . . . . . . . . . . 70

6 Air cavities at the inner cylinder of turbulent Taylor-Couette flow 71

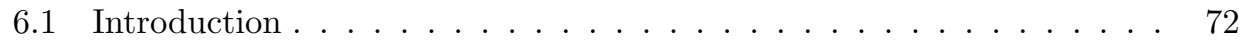

6.2 Experimental method . . . . . . . . . . . . . . . . . . 74

6.3 Results . . . . . . . . . . . . . . . . . . . . . 76

6.4 Discussion and conclusions . . . . . . . . . . . . . . . . 86

7 The influence of wall roughness on bubble drag reduction in TaylorCouette turbulence $\quad \mathbf{8 9}$

7.1 Introduction $\ldots \ldots \ldots \ldots \ldots \ldots \ldots$. . . . . . . . . . . 90

7.2 Experimental method $\ldots \ldots \ldots \ldots \ldots$. . . . . . . . . . 91

7.3 Results . . . . . . . . . . . . . . . . . . . . . . 93

7.4 Conclusions $\ldots \ldots \ldots \ldots \ldots \ldots \ldots$

$\begin{array}{ll}\text { Conclusions } & 99\end{array}$

\begin{tabular}{ll}
\hline References & 103
\end{tabular}

$\begin{array}{ll}\text { Summary } & 119\end{array}$

\begin{tabular}{ll}
\hline Samenvatting & 121
\end{tabular}

\begin{tabular}{ll}
\hline Acknowledgements & 125
\end{tabular}

\begin{tabular}{ll}
\hline About the author & 129
\end{tabular}

\begin{tabular}{ll}
\hline Publications & 131
\end{tabular} 


\section{Introduction}

In many industrial applications, the interaction between fluids and solid surfaces play an important role. Everyday examples are not hard to find: the flow around aircraft and wind turbines, the flow through a jet engine, oil transport through pipelines, and many more. Whenever fluids interact with solid walls, energy is dissipated due to skin friction and/or pressure drag. Engineers and physicists worldwide try to reduce the energy consumption of these processes, and thus the fuel costs and $\mathrm{CO}_{2}$ emissions.

Some of these processes involve multiphase flows, i.e. a mixture between multiple liquids, solid particles and/or gasses. In many multiphase flows, a dispersed phase is carried by a carrier fluid. Examples are countless, and include atmospheric flows, combustion chambers in engines, plankton in the ocean, sediment-laden rivers, mud slides, avalanches, and distillation columns. In the vast majority of these large processes, the flow is 'turbulent', a flow state which is characterised by chaotic motion of fluid, which therefore is very hard to predict or to compute. A clear example of multiphase turbulent flows are atmospheric flows: it is a multiphase flow consisting of both air and water (in liquid and solid state), and a large separation of scales: from tiny rain droplets up to $1000 \mathrm{~km}$ sized tropical cyclopes. Our knowledge of turbulent multiphase flows is limited. E.g. think of the prediction of our weather: even with the most modern techniques, we cannot predict the weather more than several days in advance.

Virtually every flow experiences wall roughness. As the intensity of the turbulence - largely governed by the 'Reynolds number' - of the flow increases, the length-scales in the flow decrease, and eventually every wall is seen by the fluid as being rough. The influence of wall roughness on the drag is a classic topic within fluid dynamics, and has been well-studied over a century from an engineering perspective. Even though this field has now become mature, open questions remain. For a limited number of systems, such as pipe flow, empirical formulas exist to calculate the friction induced by roughness, but for only slightly different situations our knowledge is lacking.

Next to wall roughness and multiphase fluid, many flows undergo transient effects. A commonly observed phenomenon is flows with time-dependent driving. The driving can be periodic, such as periodically heating from the sun, tidal waves due to periodic fluctuations in gravitational forces, and the periodic beating of our heart. A different category is fluid in which the energy input is removed, the fluid gradually comes to rest as its velocity and kinetic energy decay, such as wakes behind aircraft and ships. 
Fully understanding these processes is difficult. The large majority of studies in the field of turbulence focussed on idealized flows, which e.g. have smooth walls, are single-phase, are constantly driven, or the turbulent fluctuations are assumed to be homogeneous and isotropic in space (HIT). Analytical approaches are useful to obtain overall scaling relations, but getting a full quantitative solution is not possible. Numerical simulations will give us these full quantitative solutions, but the achievable Reynolds numbers are limited due to computational power, especially when dealing with wall roughness, transient effects or multiphase flows. Simplified numerical methods, such as RANS and LES, overcome the problem of computational costs and can be useful for engineering purposes, but their added physical insight is limited. Experimentally, even though experiments in multiphase flows are not trivial, we can measure global flow properties without too much difficulties. However, most optical measurement techniques are impossible in multiphase flows, so that local flow information, such as the velocity, is difficult to obtain.

Therefore, a single perfect method of studying these types of fluid systems does not exist. Eventually, the best understanding of the aforementioned topics will be obtained from combined theoretical, numerical and experimental efforts from physicists, mathematicians and engineers.

\section{Air lubrication and bubble drag reduction}

One particular field in which efforts are being made to reduce the overal fuel consumption is the maritime industry. Around $90 \%$ of the world trade is transported by ships [1], which means that a few percentage of fuel savings would massively impact the overall fuel consumption and costs. Drag in maritime vessels consists of three major components: skin friction, wave drag and viscous pressure drag. Wave and viscous pressure drag are minimized by optimizing the shape of the vessel [2]. It is not possible to reduce the skin friction in a similar manner, as the skin friction is proportional to the wetted surface and velocity squared. One way to reduce the skin friction is by air lubrication. In this concept, air is injected under the hull of the ship, where it forms a lubricative layer between the ships hull and the surrounding water. Various types of air lubrication are suggested, such as bubble drag reduction, air layers, air chambers and air cavities. Most straightforward to apply is bubble drag reduction, which is achieved by injecting air below the ship's hull. When excessive amounts of air are injected, an air layer will develop. An air layer significantly decreases the wetted area, and thus the friction, at the cost of large expenses for the air injection itself. With the air chamber and air cavity concepts, additional structural modifications are applied to the hull to prevent air leakage and to increase the air layer stability. Although laboratory results and some full-scale measurements are promising, the governing parameters are not yet well understood. As a result, maritime operators are somewhat reluctant to apply these techniques on their fleet.

Furthermore, biofouling, i.e. the accumulation of organisms on the hull's wetted surface is known to significantly increase the roughness and hence the drag of ves- 
sels. To which extent wall roughness influences air lubrication is not known, and the interplay between air lubrication and biofouling clearly is an area open for research. In addition, most experimental efforts on air lubrication are performed in laboratory environments with fresh, or even purified water, whereas dissolved ions and organic surfactants in the ocean alter the behaviour of air in water. The effectiveness of air lubrication in salty water, possibly in the presence of fouled surfaces is not known, and thus there is a clear need for more well-controlled measurements.

\section{Taylor-Couette turbulence}

A system which is particularly well-suited to study new concepts in fluid dynamics is the Taylor-Couette system. Taylor-Couette flow, i.e. the flow between 2 concentric, independently rotating cylinders, is one of the fundamental systems in which fluid physics is studied. It has the advantage of being a closed system, with a measurable exact energy balance between the energy input and dissipation. Due to its simple geometry, the system can be constructed with high precision. Over the last century, this system has received tremendous attention, and has been studied extensively by analytical, numerical and experimental efforts. Research areas include pattern formation, (transition to) turbulence, instabilities and viscosity measurements.

The Taylor-Couette geometry is described by an inner radius $r_{i}$, an outer radius $r_{o}$ and the height $L$ of the setup. Two geometric ratios are relevant: the radius ratio $\eta=r_{i} / r_{o}$ and the aspect ratio $\Gamma=L / d$, in which $d=r_{o}-r_{i}$ is the gap width between the cylinders. The driving of the cylinders is characterized by two Reynolds numbers: the inner Reynolds number $\operatorname{Re}_{i}=\omega_{i} r_{i} d / \nu$ and the Reynolds number of the outer cylinder $\operatorname{Re}_{o}=\omega_{o} r_{o} d / \nu$. Here, $\nu$ is the kinematic viscosity of the fluid, and $\omega_{i, o}$ is the angular velocity of the inner and outer cylinder, respectively. Alternatively, the driving can be described by the Taylor number:

$$
\mathrm{Ta}=\frac{(1+\eta)^{4}}{64 \eta^{2}} \frac{d^{2}\left(r_{o}+r_{i}\right)^{2}\left(\omega_{i}-\omega_{o}\right)^{2}}{\nu^{2}}
$$

and the rotation ratio $a=-\omega_{o} / \omega_{i}$. Only inner cylinder rotation corresponds to $a=0$, whereas $a>0$ and $a<0$ denote the counter-rotating and co-rotating regimes, respectively. Whereas the Reynolds number compares the inertial and viscous forces, the Taylor number characterises the importance of centrifugal forces relative to viscosity.

The primary response parameter of the system is the torque $\tau$ which is needed to rotate the cylinders at constant angular velocities. The torque can be non-dimensialized as a 'Nusselt number' $\mathrm{Nu}_{\omega}=\tau / \tau_{\text {lam }}$, in which $\tau_{\text {lam }}$ is the torque in the purely laminar, azimuthal flow case. In this way, the similarity between Taylor-Couette (TC) flow and Rayleigh Bénard (RB) convection are emphasised. Different dimensionless representations of the torque are $G=\tau / 2 \pi L \rho \nu^{2}$ or a friction coefficient $C_{f}=\tau /\left(L \rho \nu^{2}\left(\operatorname{Re}_{i}-\eta \operatorname{Re}_{o}\right)^{2}\right)$.

Throughout this thesis, the Twente Turbulent Taylor-Couette facility is used, which is depicted in figure 1 . In this setup, the inner and outer radii are $r_{i}=200$ 


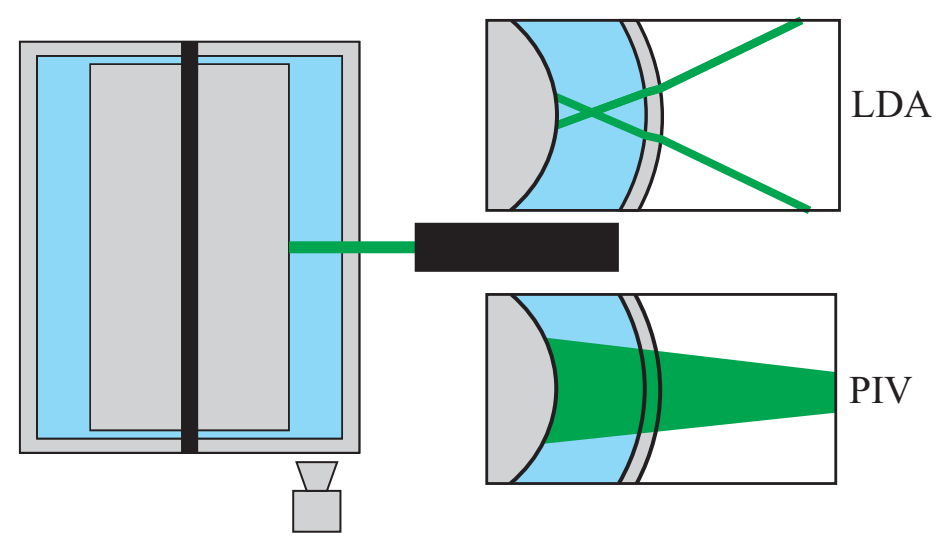

Figure 1: A sketch of the used Taylor-Couette setup. Left: cross-section of the setup. Right: used PIV and LDA setup, here depicted at mid-height.

$\mathrm{mm}$ and $r_{o}=279 \mathrm{~mm}$, respectively, giving a radius ratio of $\eta=0.71$ and a gap width of $d=79 \mathrm{~mm}$. The height of the setup is $L=927 \mathrm{~mm}$, resulting in an aspect ratio of $\Gamma=11.7$. Both cylinders can rotate independently. The maximum rotation rates of the inner and outer cylinder are $f_{i}=20 \mathrm{~Hz}$ and $f_{o}= \pm 10 \mathrm{~Hz}$, respectively. With water at $20{ }^{\circ} \mathrm{C}$, this corresponds to Reynolds numbers up to $\operatorname{Re}_{i}=2 \times 10^{6}$ and $\operatorname{Re}_{o}= \pm 1.4 \times 10^{6}$. The outer cylinder is transparent, allowing for non-intrusive optical measurements and flow visualizations. The endplates rotate with the outer cylinder. The energy dissipation of the fluid is significant, and the fluid needs to be cooled to keep the temperature, and thus the viscosity constant. Therefore, we cool the setup actively through the endplates. We refer to ref. [3] for all experimental details. The torque is measured with an internal torque transducer, which is placed in the inner cylinder. The exact relevant setup characteristics are explained in the corresponding chapter.

Besides the global torque, we are interested in studying local flow properties. To do so, we used a variety of experimental tools, such as particle image velocimetry (PIV), laser Doppler anemometry (LDA) and high-speed imaging. In contrast to e.g. pitot tubes and hot-wire anemometry, these tools are non-intrusive, meaning the flow is not disturbed by the measurement technique. With LDA, we measure the velocity at a single point at a high data-rate, using the Doppler shift of the reflected light of seeding particles. PIV is a technique with which we measure the two velocity components in a plane. With high-speed imaging, it is possible to visualize fast-moving flow dynamics which are not possible to capture by the eye or with conventional photography. Additionally, it is crucial to always measure the temperature, as the fluid viscosity (and thus all dimensionless quantities) depend on it. The temperature is measured with non-intrusive temperature sensors (PT100), which are mounted flush to the wall of the inner cylinder. 


\section{Open questions}

The majority of Taylor-Couette research focussed on statistically stationary, single phase flows with smooth walls. For this case, the torque scaling up to high Reynolds numbers is well-studied, i.e. $\mathrm{Nu}_{\omega} \propto \mathrm{Ta}^{0.4}[4]$, similar to the $\mathrm{Nu} \propto \mathrm{Ra}^{0.4}$ scaling for $\mathrm{RB}$ convection 8. Efforts are made to study the dynamics of bubbles in TC flow, although most studies focussed on lower Reynolds number regimes. For the limited number of studies in the highly turbulent regime, it was shown that a few percent of bubbles lead to a huge drag reduction, i.e. with $4 \%$ of bubbles $40 \%$ drag reduction is observed [9].

TC flow with rough walls saw limited attention by two exploratory studies 10,11 . Time-dependent driving of TC turbulence is completely unexplored. In this thesis, we attempt to answer the following questions:

- When the energy input is removed, how does the kinetic energy decay?

- How does a turbulent flow respond to periodic forcing?

- Can we understand the torque scaling in the presence of rough riblets?

- Can we unravel the mechanism behind bubble drag reduction?

- Can we use the TC system to study other forms of air lubrication, such as air cavities?

- Is bubble drag reduction still effective in the presence of rough walls?

\section{A guide through the thesis}

This thesis consists of 3 different parts, which are Part 1: Transient turbulence, Part 2: Roughness in turbulence, and Part 3: Air lubrication in turbulent flows. In Part 1, we focus on the transient effects of non-constantly driven cylinders. This is done by either completely stopping the cylinder rotation (chapter 1) or by periodically drive the cylinders (chapter 2). Then, in Part 2, we study the effects of roughness on TC flow and its energy dissipation. In chapter 3, we show that the 'asymptotic ultimate turbulence regime' can be reached, which was predicted by Robert Kraichnan for Rayleigh-Bénard convection in 1962, but now shown in Taylor-Couette flow by the use of transverse ribs. We conclude our study on roughness by studying the influence of roughness height in chapter 4 . Part 3 focusses on multiphase flows in TC flow. We study the drag-reducing effects of bubbles in chapter 5 , to understand the physical mechanism of bubble drag reduction. In chapter 6, we explore the possibilities of studying the dynamics of air cavities in TC flow. As a concluding study, we combine roughness and bubbles in chapter 7 on page 89. All conclusions and an outlook can be found in the last chapter. 


\section{Part I}

\section{Transient turbulence}




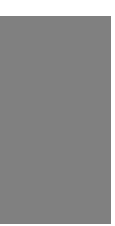




\section{Chapter 1}

\section{Self-similar decay of high Reynolds number Taylor-Couette turbulence ${ }^{T}$}

We study the decay of high-Reynolds number Taylor-Couette turbulence, i.e. the turbulent flow between two coaxial rotating cylinders. To do so, the rotation of the inner cylinder $\left(\operatorname{Re}_{i}=2 \times 10^{6}\right.$, the outer cylinder is at rest) is stopped within $12 \mathrm{~s}$, thus fully removing the energy input to the system. Using a combination of laser Doppler anemometry and particle image velocimetry measurements, six decay decades of the kinetic energy could be captured. First, in the absence of cylinder rotation, the flow-velocity during the decay does not develop any height dependence in contrast to the well-known Taylor vortex state. Second, the radial profile of the azimuthal velocity is found to be self-similar. Nonetheless, the decay of this wallbounded inhomogeneous turbulent flow does not follow a strict power law as for decaying turbulent homogeneous isotropic flows, but it is faster, due to the strong viscous drag applied by the bounding walls. We theoretically describe the decay in a quantitative way by taking the effects of additional friction at the walls into account.

\footnotetext{
${ }^{1}$ Published as: Ruben A. Verschoof, Sander G. Huisman, Roeland C.A. van der Veen, Chao Sun, and Detlef Lohse, Self-similar decay of high Reynolds number Taylor-Couette turbulence, Phys. Rev. Fluids 108, 024501(R) (2016).

Experiments by Verschoof, Huisman and van der Veen. Data analysis by Verschoof. Verschoof, Huisman and Lohse wrote the paper. Sun and Lohse supervised the project. All authors discussed the results and proofread the paper.
} 


\section{$1.1 \quad$ Introduction}

Turbulence is a phenomenon far from equilibrium: Turbulent flow is driven in one or the other way by some energy input and at the same time energy is dissipated, predominantly (but not exclusively) at the smaller scales. For statistically stationary turbulence, this balance is reflected in the famous picture of the Richardson-Kolmogorov energy cascade 12,13 . While the driving on large scales clearly is non-universal, depending on the flow geometry and stirring mechanism, the energy dissipation mechanism has been hypothesized to be self-similar 14 19].

How exactly is the energy taken out of the system? A good way to find out is to turn off the driving and follow the then decaying turbulence, as then all scales are probed during the decay process. This has been done in various studies over the last decades for homogeneous isotropic turbulence (HIT). Experimentally, the focus of attention was on grid-induced turbulence [19 26], whereas in numerical simulations periodic boundary conditions were used $27-30]$. To what degree the decay of the turbulence depends on the initial conditions [31 33] and whether or not it is selfsimilar has controversially been debated $16,22,27,34,38$. We note that for HIT, already from dimensional analysis one obtains power laws for the temporal evolution of the vorticity and kinetic energy in decaying turbulence, namely $\omega(t) \propto t^{-3 / 2}$ and $k(t) \propto t^{-2}$, respectively, in good agreement with many measurements $21,23,39$. These scaling laws are also obtained [40] when employing the "variable range mean field theory' of Ref. [41], developed for HIT. In that way, the late-time behavior, when the flow is already viscosity dominated, can also be calculated, allowing for the calculation of the lifetime of the decaying turbulence [40].

However, real turbulence is neither homogeneous nor isotropic, but it has anisotropies and is wall-bounded, with a considerable fraction of the dissipation taking place in the corresponding boundary layers. Studies on the decay of fully developed turbulence flow in wall-bounded flows are however scarce [42], though exploring the decay of such flows would teach us about the energy dissipation in the boundary layers and its possible universality. The reason for the scarcity of such studies may be that

for the most canonical and best-studied wall-bounded flow, namely, pipe flow 43 46], the decaying turbulent flow is flushed away downstream so that it is hard to study it.

This problem is avoided in confined and at the same time closed turbulent flows, such as Rayleigh-Bénard flow 4748 or Taylor-Couette (TC) flow 49 51], i.e., the flow between two independently rotating co-axial cylinders (Fig. 1.1). Indeed, turbulent TC flow is neither homogeneous nor isotropic, due to coherent structures that persist also at high Reynolds numbers 52,53, and the boundary layers play the determining role in the angular momentum transfer from the inner to the outer cylinder [54,55].

In this chapter, we employ the TC system to study the temporal and spatial behavior of decaying confined and wall-bounded turbulence, and compare it with the known results for HIT, thus complementing the study of Ref. 19 for decaying homogeneous isotropic turbulence. We suddenly stop the inner cylinder rotation (similarly as in Ref. [56], which focused on the decay of turbulent puffs for much lower Reynolds number) and then measure the velocity field over time. We find that 
the decay models for HIT [19, 40] are insufficient to describe the data, but when extending them by explicitly taking the wall friction into consideration, the measured data can be well described. Though the decay does not follow a power law due to the wall friction, the velocity profiles are still self-similar. In the study we restrict ourselves to fixed outer cylinder and decaying flow; for a numerical study on flow stabilization by a corotating outer cylinder we refer the reader to Ref. [57].

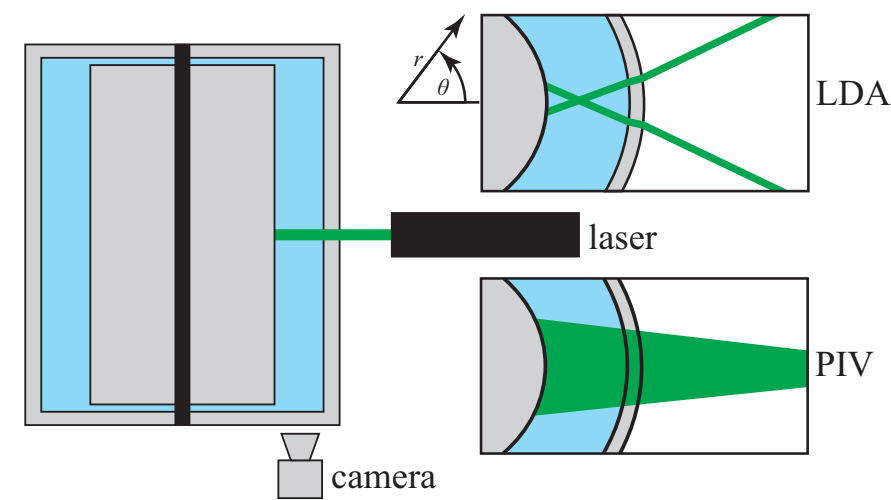

Figure 1.1: Schematic of the vertical cross-section of the $\mathrm{T}^{3} \mathrm{C}$ facility. The laser beams are in the horizontal plane $(r, \theta)$ at midheight; $z=L / 2$ (unless stated otherwise) for both the LDA and PIV measurements. The top right inset shows the horizontal cross-section, showing the LDA beams (not to scale). The beams refract twice on the OC and intersect at the middle of the gap $\left(r=r_{m}\right)$, giving the local velocity component $u_{\theta}$. The bottom right inset shows, for the PIV measurements, particles are illuminated by a thin laser sheet. We use the viewing windows in the end plate to look at the flow from the bottom, thus obtaining the velocity components $u_{\theta}$ and $u_{r}$ in the $(r, \theta)$ plane.

\subsection{Experiments}

The experiments were performed at the Twente Turbulent Taylor-Couette facility $\left(\mathrm{T}^{3} \mathrm{C}\right)[3$, consisting of two independently rotating concentric smooth cylinders. The setup has an inner cylinder (IC) with a radius of $r_{i}=200 \mathrm{~mm}$ and an outer cylinder (OC) with a radius of $r_{o}=279 \mathrm{~mm}$, giving a mean radius $r_{m}=\left(r_{i}+r_{o}\right) / 2=239.5$ $\mathrm{mm}$, a radius ratio of $\eta=r_{i} / r_{o}=0.716$ and a gap width $d=r_{o}-r_{i}=79 \mathrm{~mm}$. The IC can rotate up to $f_{i}=20 \mathrm{~Hz}$, resulting in a Reynolds number up to $\operatorname{Re}_{i}=2 \pi f_{i} r_{i} d / \nu=$ $2 \times 10^{6}$ with water as the working fluid at $T=20^{\circ} \mathrm{C}$. The cylinders have a height of $L=927 \mathrm{~mm}$, giving an aspect ratio of $\Gamma=L / d=11.7$. The transparent acrylic OC allows for non-intrusive optical measurements. The end plates, which are partly transparent, are fixed to the OC. The velocity is measured using two non-intrusive optical methods: particle image velocimetry (PIV) and laser Doppler anemometry (LDA), as shown in Fig. 1.1. The LDA measurements give the azimuthal velocity 
$u_{\theta}(t)$ at mid-gap $\left(r=r_{m}\right)$ and at several heights. The water is seeded with $5 \mu \mathrm{m}$ diameter polyamide tracer particles with a density of $1.03 \mathrm{~g} / \mathrm{cm}^{3}$. The laser beams are focused in the middle of the gap, i.e. at $r=r_{m}$. Using numerical ray-tracing, the curvature effects of the OC are accounted for [58]. The PIV measurements are performed in the $\theta-r$ plane at mid-height $(z=L / 2)$, using a high-resolution camer $2^{2}$ operating at $20 \mathrm{~Hz}$. The spatial resolution of the PIV measurements is $0.04 \mathrm{~mm} /$ pixel, with interrogation windows of 32 pixel $\times 32$ pixel. The flow was illuminated from the side with a pulsed Nd:YLF laser ${ }^{3}$, with which a horizontal light sheet is created (fig. 1.1). The water was seeded with $20 \mu \mathrm{m}$ polyamide tracer particles. Because of the large velocity range of our measurements, several measurements with a changing $\Delta t$ are performed (50 $\mathrm{s} \leq \Delta t \leq 50 \mathrm{~ms})$, so that the entire velocity range is fully captured. The PIV measurements were processed to give both the radial velocity $u_{r}(\theta, r, t)$ and azimuthal velocity $u_{\theta}(\theta, r, t)$. The Stokes number of the seeding particles are always smaller than $\mathrm{St}=\tau_{p} / \tau_{\eta}<0.2$, so the particles faithfully follow the flow 13,59 .

We first drive the turbulence at a rotation rate of $f_{i}=20 \mathrm{~Hz}\left(\operatorname{Re}_{i}=2 \times 10^{6}\right)$ of the $\mathrm{IC}$, while the $\mathrm{OC}$ is at rest, allowing for the development of a statistically stationary state. We then decelerated the IC within approximately $12 \mathrm{~s}$ linearly down to $f_{i}=0$ $\mathrm{Hz}$, so, starting from $t=0 \mathrm{~s}$, there is zero energy input. The deceleration rate is limited by the braking power of the electric motor. The deceleration time is much smaller than the typical time scale for turbulence decay $\left(\tau=d^{2} / \nu \approx 6 \times 10^{3} \mathrm{~s}\right)$. The velocity measurements start when the IC has come to rest, so at $t=0, f_{i}=f_{o}=0$ $\mathrm{Hz}$.

\subsection{Results and analysis}

In Fig. 1.2, the azimuthal velocity decay $u_{\theta}(t)$ is shown. The results obtained with PIV and LDA are the same; the LDA results only start to deviate from the PIV measurements when the measured velocities are close to the dynamic range of the LDA system. Viscous friction dissipates the energy, bringing the fluid eventually to rest. Also the spatial velocity fluctuations, characterized by the standard deviation of the azimuthal velocity fluctuations $\sigma_{u_{\theta}}(t)$ decay in a very similar way, see Figs. 1.2 and 1.4. The LDA data (dashed lines shown in fig. 1.2) are measured at different heights using LDA; their collapse indicates that during the decay no Taylor rolls develop, which would lead to a height-dependence of the profiles. This is in contrast to TC flow with increasing inner cylinder rotation, where with increasing $\mathrm{Re}_{i}$ first Taylor rolls develop [60, before one arrives at the structureless fully developed turbulent state (for the chosen geometry) 7, 51]. The reason for this difference is that in the constantly rotating case angular momentum is transported from the inner to the outer cylinder, whereas in the decaying case the angular momentum is transported from the bulk to both walls, i.e., a net momentum transport between the cylinders is absent.

\footnotetext{
${ }^{2}$ pco, pco.edge camera, double frame sCMOS, 2560 pixel $\times 2160$ pixel resolution, operated in dual frame mode.

${ }^{3}$ Litron, LDY303HE Series, dual-cavity, pulsed Nd:YLF PIV Laser System. The sheet thickness was approximately $1 \mathrm{~mm}$.
} 


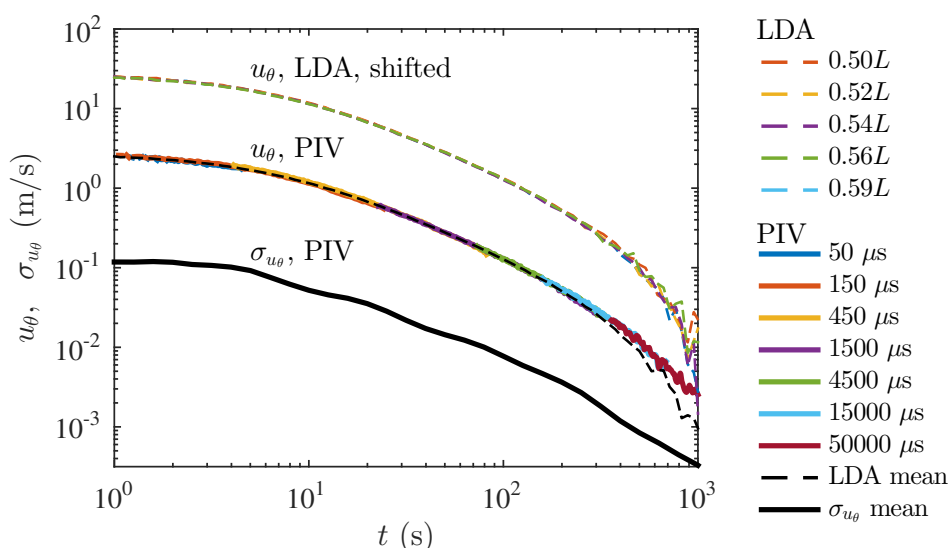

Figure 1.2: Midgap azimuthal velocity as a function of time at mid-height. PIV measurements with seven different interframe times $\Delta t$ were performed, as shown in the legend, to produce accurate results over the entire velocity range. The PIV measurements are averaged azimuthally and radially; we average over $r_{m}-4 \mathrm{~mm}<$ $r<r_{m}+4 \mathrm{~mm}$, corresponding to $10 \%$ of the gap width. These results are confirmed by LDA measurements performed at several heights (dashed lines), which are shifted by one decade for clarity. The standard deviation $\sigma_{u_{\theta}}$, a measure for the spatial velocity fluctuations, is shown as the solid black line. The data are averaged azimuthally and radially as described above, and binned using logarithmic bins of 0.1 decades. The measurements cover three orders of magnitude of the velocity, corresponding to six orders of magnitude in kinetic energy. The measurement uncertainty roughly corresponds to the width of the lines.

From earlier work [54], we know that the normalized velocity profiles in the bulk are nearly Re independent and height independent over a large range of Reynolds numbers. Here we focus on the bulk flow velocity where the Re-independence holds. Correspondingly, we would get similar results for different Re-measurements in the turbulent regime. The axial and radial velocities are approximately 50-100 times smaller than the azimuthal velocity, so their contributions to the total kinetic energy are negligible.

Turbulence is characterized by a fluid motion over a large range of length scales. In TC flow, the upper limit is the gap width $d$ and the smallest length scale is the Kolmogorov scale $\eta_{K}$, which is defined as $\eta_{K}=\left(\nu^{3} / \epsilon\right)^{1 / 4}$. From the bulk velocity as measured with PIV, we calculate the energy dissipation rate from the change in velocity over time, i.e. $\epsilon=d\left(\frac{1}{2} u_{\theta}^{2}\right) / d t$. As shown in Fig. 1.3. as the velocity decreases, also the energy dissipation rate becomes smaller. Clearly, the dissipative length scale changes over time, though $\eta_{K}$ only remains a fraction of the gap. Consequently, we cannot faithfully resolve spatial gradients in the flow with our PIV data.

To compare the experimental data on the decay of the velocity and their fluctuations with theory, we first define the respective Reynolds numbers, namely $\operatorname{Re}_{u_{\theta}}(t)=$ 

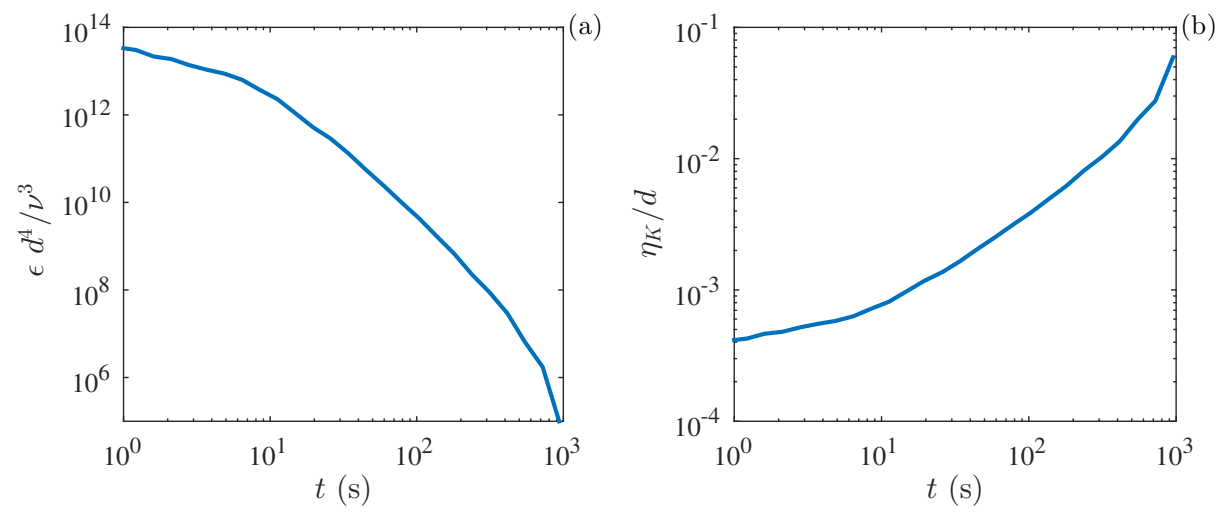

Figure 1.3: (a) Energy dissipation rate, normalized with $\nu^{3} / d^{4}$, as a function of time. Here $\epsilon$ is calculated from the PIV data as shown in Fig. 1.2. $\epsilon$ drops by more than 8 orders of magnitude. (b) Kolmogorov length scale $\eta_{K}$ as a function of time, normalized with the gap width $d$. As time progresses, the separation of scales becomes smaller, although $\eta_{K}$ remains small.
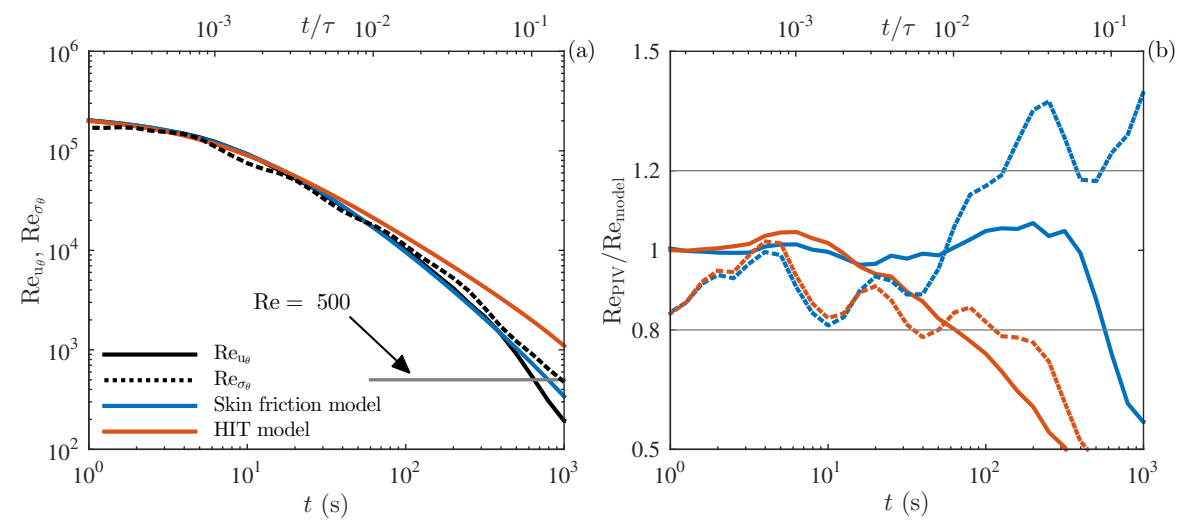

Figure 1.4: (a) The PIV results for $\operatorname{Re}_{u_{\theta}}$ and $\mathrm{Re}_{\sigma_{\theta}}$, binned using logarithmic bins of 0.1 decades: The scale on the $\mathrm{y}$-axis refers to $\operatorname{Re}_{u_{\theta}}$, whereas the one for $\operatorname{Re}_{\sigma_{\theta}}$ is vertically shifted to show that the decay of the spatial fluctuations and the mean is the same. On the horizontal axis, both the real time and the non-dimensionalized time (normalized by $\tau=d^{2} / \nu$ ) are shown. Included in the graph are the results for the HIT model of Ref. [40] and those of the Prandtl-von Kármán skin friction model (eq. (2)), which includes the effects of the walls. Below the short line at $\operatorname{Re}=500$, thermal effects set in. (b) Ratio between the measurements and respectively the HIT model (solid red line) and the skin friction model (solid blue line). The respective dashed lines show the ratio between the fluctuation decay and the two models. 
$u_{\theta}\left(r=r_{m}, t\right) d / \nu$ taken at mid-gap $r_{m}$ and $\operatorname{Re}_{\sigma_{\theta}}(t)=$ const $\times \sigma_{u_{\theta}}\left(r=r_{m}, t\right) d / \nu$ for the fluctuations, which we have rescaled with a constant so that it collapses with $\operatorname{Re}_{u_{\theta}}$ at $t=0$, i.e. const $=\operatorname{Re}_{u_{\theta}}(0) /\left(\sigma_{u_{\theta}}(0) d / \nu\right)$. The curves show that the decay of the velocity itself and the fluctuations is the same [see Fig. 1.4 $]$ ]. We then compare the decay of $\operatorname{Re}(t)$ with the one predicted for the theory of HIT, as it follows from a numerical integration of the ordinary differential equation obtained in the model of Ref. [40],

$$
\dot{\mathrm{Re}}=-\frac{1}{3} \frac{\nu}{d^{2}} c_{\mathrm{HIT}}(\mathrm{Re}) \operatorname{Re}^{2},
$$

with $c_{\mathrm{HIT}}(\mathrm{Re})$ given by Eq. (6) of [40]. From Fig. 1.4 we see that, though in the beginning the decay is reasonably well described, at a later time the decay experimentally found in this wall-bounded flow is much faster than resulting from the model for HIT.

We therefore replace the model for $c_{\mathrm{HIT}}(\mathrm{Re})$ in Eq. (1.1) by a model for wallbounded flow, namely by a friction factor $c_{f}(\mathrm{Re})$ following from the Prandtl-von Kármán skin friction law 13,43,53,

$$
\frac{1}{\sqrt{c_{f}}}=a \log _{10}\left(\operatorname{Re} \sqrt{c_{f}}\right)+b .
$$

For pipe flow, a good description of various experimental data can be achieved with $a=1.9$ and $b=-0.361]$. These values depend on the boundary conditions of the flow, i.e. on the geometry and whether or not the flow is actively driven or decaying. For decaying turbulence in the TC geometry we find that a good leastsquare fit of this model to the decay of $\operatorname{Re}(t)$ is achieved with $a=2.72$ and $b=-2.22$ (see Fig. 1.4). As can be seen, due to the extra friction in the wall regions the decay is now faster than the decay observed in HIT and much better and longer agrees with the experimental data, reflecting that the no-slip boundary conditions force the fluid to slow down faster.

How long do the respective models for HIT [40] and the Prandtl-van Karman skin friction hold? We define the beginning of the discrepancy between data and models to be $\left|1-\operatorname{Re}_{\mathrm{PIV}} / \operatorname{Re}_{\text {model }}\right|=0.2$, which is visible as thin gray lines in Fig. 1.4(b). From this definition, we calculate that the discrepancy between the $\operatorname{Re}_{u_{\theta}}$ and the HIT model starts at $t=45 \mathrm{~s}$ and the one between $\operatorname{Re}_{u_{\theta}}$ and the friction model at $t=530$ s.

As was discussed in the introduction, self-similarity is commonly assumed and observed [16, 20] in the decay of HIT flows. The question is whether self-similar flow fields still exist in the decay of inhomogeneous wall-bounded turbulence with a strong shear. By analyzing several instantaneous velocity profiles (see fig. 1.5), we found that also for this inhomogeneous turbulence the normalized velocity profiles are selfsimilar during the decay, see Fig. 1.6. We find that the normalized velocity profile is self-similar up to $t \approx 400 \mathrm{~s}$. Hitherto, a self-similar decay has not yet been observed for wall-bounded inhomogeneous turbulence, and it is remarkable that also in this highly inhomogeneous and anisotropic flow a self-similar decay exists. Eventually the 

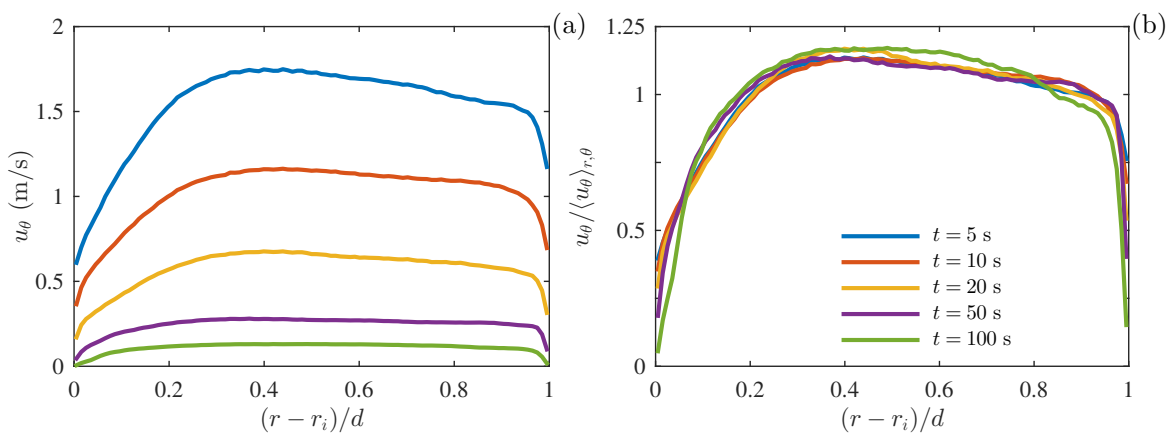

Figure 1.5: Azimuthal velocity profiles $u_{\theta}(r)$ for $z=L / 2$, averaged over $\theta$. The decelerating effects of the walls (left and right edges of the figure) can be seen clearly. (b) The velocity is normalized with the (spatial) mean azimuthal velocity, $\left\langle u_{\theta}\right\rangle_{r, \theta}(t)$. The normalized velocity profiles overlap, indicating the self-similarity of the velocity profile during the decay.

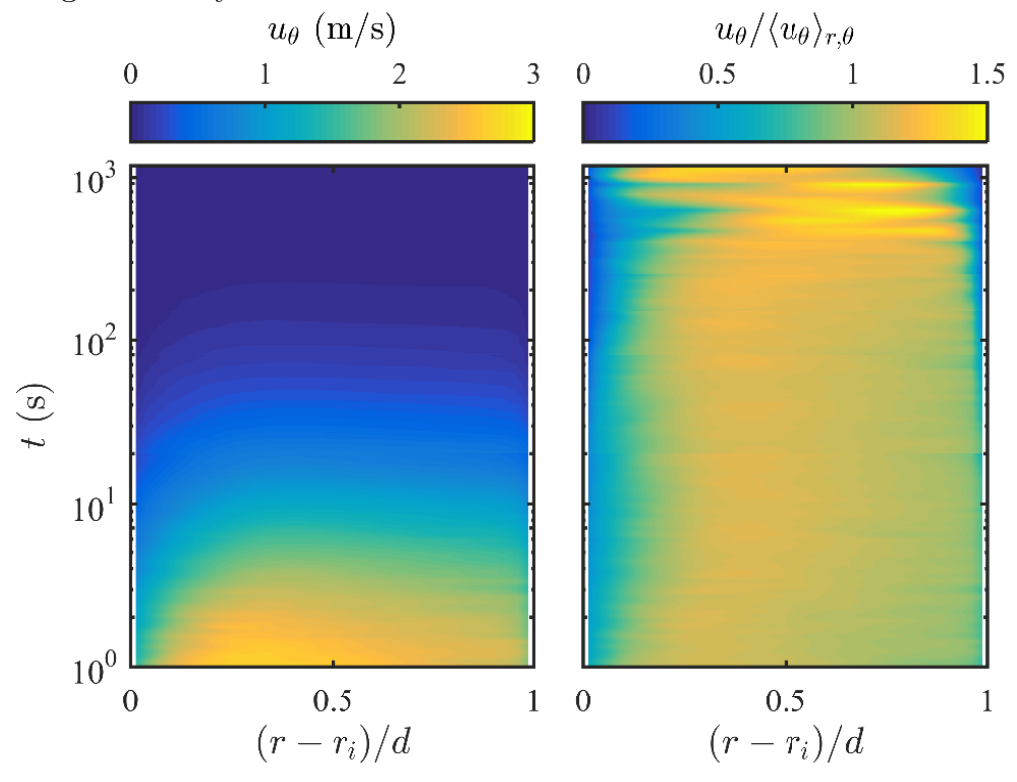

Figure 1.6: (a) Measured velocity $u_{\theta}$ as a function of time, resulting from seven PIV measurements with changing $\Delta t$ and averaged over $\theta$. (b) Same results as shown in (a) but now normalized with the mean velocity $\left\langle u_{\theta}(t)\right\rangle_{r, \theta}$. The normalized velocity is self-similar up to $t \approx 400 \mathrm{~s}$. 
self-similarity breaks down, possibly due to thermal convection. Residual cooling in the end plates causes small temperature differences and thus thermal convection is estimated to start from $\mathrm{Re} \approx 500$ (see Ref. 3 for a detailed discussion). Therefore, the results after $t \approx 600 \mathrm{~s}$ are dominated by effects other than the initial velocity and the decay process. As can be seen in Fig. 1.4 this roughly coincides with the moment the model starts to deviate from our measurements.

\subsection{Conclusions}

In conclusion, we measured six decades of the decaying energy in Taylor-Couette flow after the cylinders were halted. During the decay, no height dependence of the flow develops, which is in contrast to the upstarting case, in which the wellknown Taylor vortices develop. The azimuthal velocity profile was found to be selfsimilar. Nonetheless, the kinetic energy in this wall-bounded flow decays faster than observed for homogeneous isotropic turbulent flows. This accelerated decay is due to the additional friction with the walls. We successfully modeled this accelerated decay by using a friction coefficient in which the Prandtl-von Kármán skin friction law for wall-bounded flow is used to model $c_{f}(\mathrm{Re})$. With this model, both the decay of the mean and the fluctuations could be described successfully. We hope that this work will stimulate further investigations into the decay of wall-bounded (and thus nonsotropic and inhomogeneous) turbulence in other flow geometries, disentangling the universal and non universal features. 


\section{Chapter 2}

\section{Periodically driven Taylor-Couette turbulence ${ }^{1]}$}

We study periodically driven Taylor-Couette turbulence, i.e. the flow confined between two concentric, independently rotating cylinders. Here, the inner cylinder is driven sinusoidally while the outer cylinder is kept at rest (time-averaged Reynolds number is $\operatorname{Re}_{i}=5 \times 10^{5}$ ). Using particle image velocimetry (PIV), we measure the velocity over a wide range of modulation periods, corresponding to a change in Womersley number in the range $15 \leq$ Wo $\leq 114$. To understand how the flow responds to a given modulation, we calculate the phase delay and amplitude response of the azimuthal velocity.

In agreement with earlier theoretical and numerical work, we find that for large modulation periods the system follows the given modulation of the driving, i.e. the system behaves quasi-stationary. For smaller modulation periods, the flow cannot follow the modulation, and the flow velocity responds with a phase delay and a smaller amplitude response to the given modulation. If we compare our results with numerical and theoretical results for the laminar case, we find that the scalings of the phase delay and the amplitude response are similar. However, the local response in the bulk of the flow is independent of the distance to the modulated boundary. Apparently, the turbulent mixing is strong enough to prevent the flow from having radius-dependent responses to the given modulation.

\footnotetext{
${ }^{1}$ Ruben A. Verschoof*, Arne K. te Nijenhuis*, Sander G. Huisman, Chao Sun, and Detlef Lohse, Periodically driven Taylor-Couette turbulence, accepted for publication at J. Fluid Mech. Verschoof and te Nijenhuis contributed equally to this work. Experiments by Verschoof and te Nijenhuis. Data analysis by te Nijenhuis. Verschoof wrote the paper. Huisman, Sun and Lohse supervised the project. All authors discussed the results and proofread the paper.
} 


\section{$2.1 \quad$ Introduction}

Periodically driven turbulent flows are omnipresent. Well-known examples include blood flow driven by the beating heart, the flow in internal combustion engines, the earth's atmosphere which is periodically heated by the sun, and tidal currents caused by periodic changes in the gravitational attraction of both the moon and sun.

One line of research assumes homogeneous isotropic turbulence. These studies focussed on the global response of the system, i.e. the response amplitude and the phase shift of the quantities such as a global Reynolds number 62], or the total energy in the system 63. Most numerical studies in addition only used simplified models, such as the GOY shell model or the reduced wave vector set approximation (REWA) 64 66. Only a limited number of DNS studies have been performed in this field, because of the computational costs needed to achieve both fully developed turbulence and sufficient statistical convergence with temporal dependence 67,69$]$. Also studies on periodically driven wind tunnels were performed 70].

The field of pulsating pipe flow received significantly more attention, presumably because of its clear industrial and biophysical relevance, see e.g. refs. [71 75], and many others. In most studies, like in the present study, an oscillatory flow was superimposed on a steady current. Depending on the relative strength, the system was either 'current-dominated' or, for strong oscillations, 'wave-dominated', the majority of the studies being current-dominated [76]. For many cases it was found that pulsations increase the critical Reynolds number [77 78], and, an initially turbulent flow can relaminarize when a periodic forcing is applied 72,79$]$. In most studies the Reynolds number of the imposed oscillatory flow however was close to the laminar-turbulent transition [74], thus, even if the steady current was fully turbulent, the oscillation was not.

Periodically driven turbulence also includes studies in a number of different wellknown and canonical closed-flow geometries, such as Rayleigh-Bénard convection 80 , 81, and von Kármán flow [10. In these systems the forcing was periodically varied over time, with the variations being of $O(10 \%)$ of either the average forcing or the energy input.

The main observations made in the studies on sinusoidal driven turbulence were similar regarding the global response of the system 10,63, 65, 68, 82. The periodic driving is governed by the Womersley number $\mathrm{Wo}_{0}=L \sqrt{\Omega} / \nu$, which can be seen as the square root of the dimensionless modulation frequency. Here, $L$ is a characteristic length-scale, $\nu$ the kinematic viscosity, and $\Omega$ the angular oscillation frequency. In the limit of extremely small Womersley numbers, the flow can fully respond to the changes, meaning that the flow behaves quasi-stationary. In this regime, no phase delay $\Phi_{\text {delay }}$ between the response and the modulation is observed, and the response amplitude is identical to the modulation amplitude. As the Womersley number is increased, the fluid system cannot follow the changing BC: the response amplitude decreases and a phase delay between input and response is observed. In the extreme case of infinite Womersley numbers, the response amplitude vanishes and a phase delay can no longer be defined. 
In this chapter, we study the physics of periodically driven turbulence in a TaylorCouette (TC) apparatus, employing a sinusoidally driven inner cylinder. TC flow, i.e. the flow of a fluid confined in the gap between two concentric cylinders, is one of the canonical systems in which the physics of fluids is studied, see e.g. the recent reviews by [50 and [51. It has the advantage of being a closed system with an exact global energy balance []eck07b, and due to its simple geometry TC systems can be accessed experimentally with high precision.

An important difference between pipe flow and TC flow is the way the system is driven. Pulsating pipe flow is driven by a time-dependent pressure difference applied to the system, but the walls remain fixed. Therefore, momentum is transported from the bulk flow to the boundary layers. In TC flow, the (periodic) driving is by the rotation of the cylinders, so that the momentum is transported from the boundary layer to the bulk flow. By periodically driving the inner cylinder we directly modulate the boundary layer, which transports the modulations to the bulk flow, whereas in pipe flow the bulk flow is directly modulated by the applied pressure gradient. Therefore, studying periodically driven Taylor-Couette turbulence sheds light on the role of the boundary layers in transporting these modulations. Further important differences are the presence of curvature effects and centrifugal forcing in TC, which are clearly absent in pipe flow. Apart from several recent studies which focussed on the decay of turbulent TC flow [57, 144, 184, or time-dependent driving close to the low Reynolds number Taylor-vortex regime [56, 183, 187, 191, 192, to our knowledge no work has been conducted so far on TC turbulence with time-dependent driving.

The outline of this chapter is as follows. We start by explaining the experimental method in $\S 2.2$. The results, in which we present the response of the flow, are shown in $§ 2.3$. Finally, we conclude this chapter in $\S 2.4$.

\section{$2.2 \quad$ Method}

In this chapter, we restrict ourselves to the case of inner cylinder rotation, while keeping the outer cylinder at rest. The inner cylinder rotation is set to

$$
f_{i}(t)=\left\langle f_{i}\right\rangle_{t}(1+e \sin (2 \pi t / T)),
$$

in which $f_{i}(t)$ is the rotation rate of the inner cylinder at time $t$ and $T=2 \pi / \Omega$ is the period of the modulation. The time $t$ is related to the phase $\Phi$ by $\Phi=2 \pi t / T$. We here chose to study the current-dominated regime. To do so, the modulation amplitude is set to $e=0.10$ throughout this work, so that the mean flow is one order of magnitude larger than the induced modulation. The time-averaged rotation rate $\left\langle f_{i}\right\rangle_{t}$ is set to $\left\langle f_{i}\right\rangle_{t}=5 \mathrm{~Hz}$, resulting in a time-averaged Reynolds number of $\left\langle\operatorname{Re}_{i}\right\rangle_{t}=$ $\left\langle u_{i}\right\rangle_{t} d / \nu=2 \pi\left\langle f_{i}\right\rangle_{t} r_{i} d / \nu=5 \times 10^{5}$. In this equation, $u_{i}=2 \pi f_{i} r_{i}$ equals the velocity of the inner cylinder with radius $r_{i}, \nu$ is the kinematic viscosity and $d$ is the gap width between the cylinders. Here, we are in the so-called 'ultimate turbulence' regime, in which both the bulk flow and boundary layers are fully turbulent $8,185,186,219]$. The 

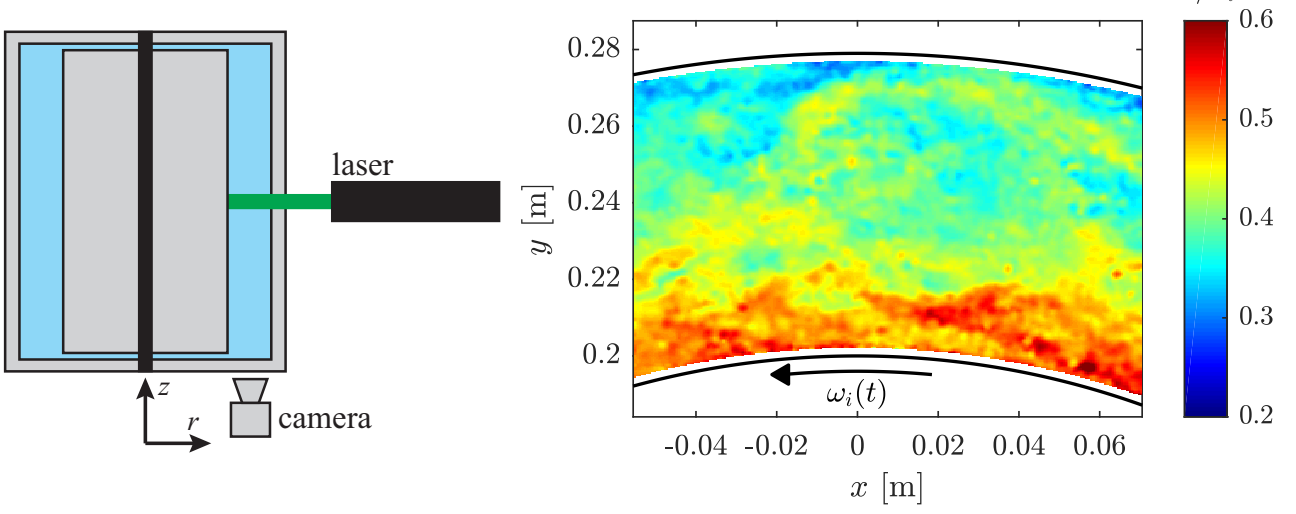

Figure 2.1: Schematic of the vertical cross-section of the $\mathrm{T}^{3} \mathrm{C}$ facility. The laser illuminates a horizontal plane $(r, \theta)$ at midheight $(z=l / 2)$ for all PIV measurements. The flow is imaged from the bottom with a high resolution sCMOS camera to obtain the velocity components $u_{\theta}$ and $u_{r}$ in the $(r, \theta)$ plane. On the right we show a typical instantaneous flow field, as measured with PIV. Here we show $u=\sqrt{u_{r}^{2}+u_{\theta}^{2}}$ normalized with the inner cylinder velocity $u_{i}$, for the case with Wo $=44.3, \Phi=2.17$ radians and an instantaneous Reynolds number of $\operatorname{Re}_{i}=5.4 \times 10^{5}$.

strength of the modulation, which can be estimated as $\Delta \operatorname{Re}_{i} \equiv e\left\langle\operatorname{Re}_{i}\right\rangle_{t}=5 \times 10^{4}$, is such that the system is well in the ultimate regime at all times. We varied the modulation period $T$ from $180 \mathrm{~s}$ down to $3 \mathrm{~s}$. The modulation period can be made dimensionless, resulting in the Womersley number, which is defined as

$$
\mathrm{Wo}=d \sqrt{2 \pi /(T \nu)} .
$$

See table 2.1 for all experimental parameters. The Womersley number is connected with the Stokes boundary layer thickness $\delta=2 \pi \sqrt{2 \nu T /(2 \pi)}$, which, in its dimensionless form $\tilde{\delta}=\delta / d=\sqrt{8} \pi /$ Wo, is proportional to the inverse of the Womersley number. The modulation frequency was limited by the power of the motor needed to accelerate and decelerate the mass of the inner cylinder $(160 \mathrm{~kg})$. Due to vibrations in the system, higher order statistics cannot be measured. We then simultaneously measured the rotational speed of the inner cylinder $f_{i}(t)$ and the fluid velocity by using non-intrusive Particle Image Velocimetry (PIV).

The experiments were performed in the Twente Turbulent Taylor-Couette $\left(\mathrm{T}^{3} \mathrm{C}\right)$ facility [3], as shown schematically in figure 2.1. The apparatus has an inner cylinder with a radius of $r_{i}=200 \mathrm{~mm}$ and a transparent outer cylinder with a radius of $r_{o}=279.4 \mathrm{~mm}$, resulting in a radius ratio of $\eta=r_{i} / r_{o}=0.716$, a gap width $d=$ $r_{o}-r_{i}=79.4 \mathrm{~mm}$. The height of the setup is $l=927 \mathrm{~mm}$, giving an aspect ratio of $\Gamma=l / d=11.7$. As working fluid we use water with a temperature of $T=20{ }^{\circ} \mathrm{C}$, which is kept constant within $0.2 \mathrm{~K}$ by active cooling through the end-plates of the setup. More experimental details of this facility can be found in 3 . 


\begin{tabular}{ccccc}
$\left\langle\operatorname{Re}_{i}\right\rangle_{t}$ & $\Delta \operatorname{Re}_{i}$ & $T[\mathrm{~s}]$ & Wo & $\tilde{\delta}$ \\
\hline $5 \times 10^{5}$ & $5 \times 10^{4}$ & 3 & 114.3 & 0.078 \\
$5 \times 10^{5}$ & $5 \times 10^{4}$ & 5 & 88.6 & 0.100 \\
$5 \times 10^{5}$ & $5 \times 10^{4}$ & 10 & 62.6 & 0.142 \\
$5 \times 10^{5}$ & $5 \times 10^{4}$ & 20 & 44.3 & 0.201 \\
$5 \times 10^{5}$ & $5 \times 10^{4}$ & 30 & 36.2 & 0.246 \\
$5 \times 10^{5}$ & $5 \times 10^{4}$ & 60 & 26.6 & 0.348 \\
$5 \times 10^{5}$ & $5 \times 10^{4}$ & 90 & 20.9 & 0.426 \\
$5 \times 10^{5}$ & $5 \times 10^{4}$ & 180 & 14.8 & 0.602
\end{tabular}

Table 2.1: Experimental details of the measurements. In all measurements the timeaveraged Reynolds number as well as the modulation strength as kept identical. By changing the modulation period $T$, we consequently change the Womersley number Wo. In the last column, we show the normalized Stokes boundary layer thickness $\tilde{\delta}=\delta / d$.

The PIV measurements were performed in the $r-\theta$ plane at mid-height $(z=l / 2)$ using a high-resolution camera operating at $15 \mathrm{fps}$ (pco.edge camera, double frame sCMOS, $2560 \times 2160$ pixel resolution). We illuminate the flow from the side with a horizontal laser sheet, as shown in figure 2.1. The used laser is a pulsed dualcavity $532 \mathrm{~nm}$ Quantel Evergreen $145 \mathrm{Nd}$ :YAG laser. We seeded the water with 1-20 $\mu \mathrm{m}$ fluorescent polyamide particles. We calculate the Stokes number which equals $S t k=\tau_{p} / \tau_{\eta}=0.0019 \ll 1$. Furthermore, the mean particle radius is roughly 6 times smaller than our Kolmogorov length scale, thus we can be sure that the particles faithfully follow the flow. The images are processed with interrogation windows of 32 $\times 32$ pixel with $50 \%$ overlap, resulting in $u_{\theta}(r, \theta, t)$ and $u_{r}(r, \theta, t)$. We were unable to measure close to the cylinders due to the strong laser light reflections.

To compare our experiments in highly turbulent flow with the laminar case, we numerically solved the response of the flow. We therefore solved the partial differential equation

$$
\frac{\partial u_{\theta}}{\partial t}=\nu\left[\frac{1}{r}\left(\frac{\partial}{\partial r}\left(r \frac{\partial u_{\theta}}{\partial r}\right)\right)-\frac{u_{\theta}}{r^{2}}\right],
$$

which is the time-dependent Navier-Stokes equation in cylindrical coordinates for the azimuthal direction under the assumptions of i) no azimuthal and axial derivatives, ii) $u_{r}=0$ and $u_{z}=0$, so that $\vec{u}(r, \theta, z, t)=u_{\theta}(r, t) \hat{e}_{\theta}$. As initial condition we used the steady-state laminar flow profile, i.e. $u_{\theta}(r, t=0)=\frac{1}{1-\eta^{2}}\left(\frac{r_{i}^{2} \omega_{i}}{r}-\omega_{i} \eta^{2} r\right)$. As time-dependent boundary conditions we set $u\left(r_{i}, t\right)=\omega_{i} r_{i}(1+0.1 \sin (2 \pi t / T))$, and the outer cylinder is stationary, i.e. $u\left(r_{o}, t\right)=0$. We run the computation for 40 periods, so that all transient effects are gone. 

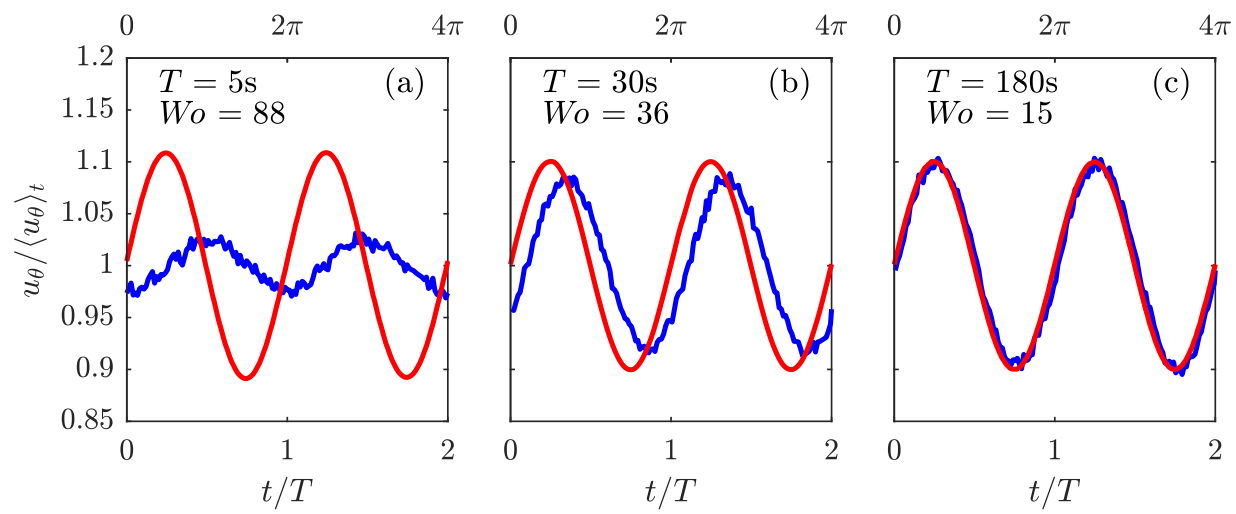

Figure 2.2: — Normalized azimuthal velocity of the sinusoidally driven inner cylinder $u_{i} /\left\langle u_{i}\right\rangle_{t}$. - Normalized azimuthal velocity $u_{\theta} /\left\langle u_{\theta}\right\rangle_{t}$ at mid-gap. Three Womersley numbers are shown, namely (a) Wo $=88$, (b) $\mathrm{Wo}_{0}=36$, and (c) Wo $=15$. The velocity is radially averaged between $0.3 \leq \tilde{r} \leq 0.7$. On the top x-axis, we show the phase $\Phi$ of the modulations in radians.

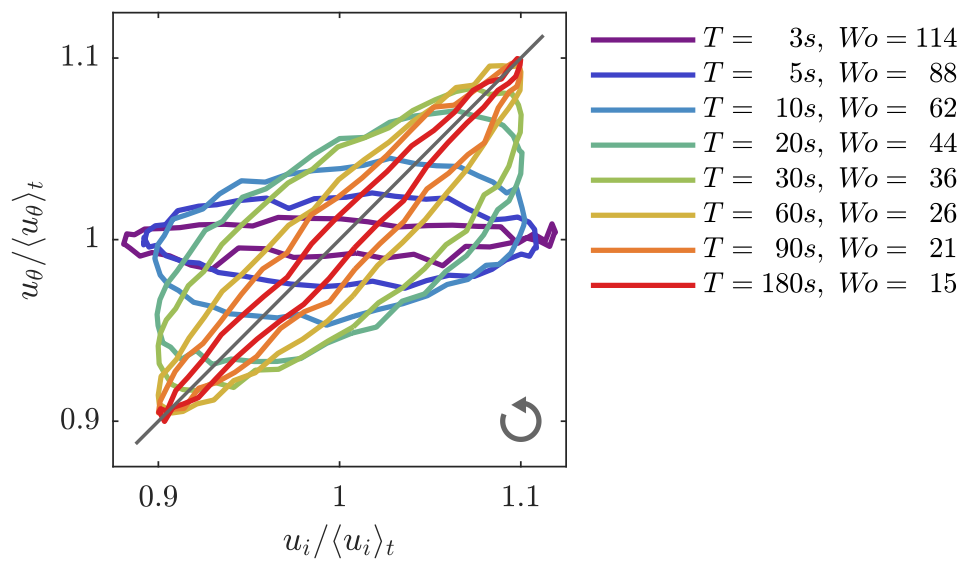

Figure 2.3: Phase averaged normalized azimuthal mid-gap flow velocity $u_{\theta} /\left\langle u_{\theta}\right\rangle_{t}$ as a function of normalized driving velocity of the inner cylinder $u_{i} /\left\langle u_{i}\right\rangle_{t}$. We show the result for all measured Womersley numbers Wo. The velocity is radially averaged between $0.3 \leq \tilde{r} \leq 0.7$. The solid grey line corresponds to the quasi-stationary case $u_{\theta} /\left\langle u_{\theta}\right\rangle_{t}=u_{i} /\left\langle u_{i}\right\rangle_{t}$. The arrow at the bottom right indicates the direction of the cycles. 


\subsection{Results and analysis}

\subsubsection{Velocity response}

In figure 2.2 we show the normalized driving and response of the mid-gap flow velocity $u_{\theta}(\tilde{r}=0.5, t)$ for three different modulation periods. The radius is nondimensionalized as $\tilde{r}=\left(r-r_{i}\right) / d$, so that $\tilde{r}=0$ corresponds to the inner cylinder and $\tilde{r}=1$ to the outer one. We non-dimensionalize both velocities by their time-averaged value, so both lines meander around 1 . For all oscillation periods, the mid-gap flow velocity oscillates with the same period $T$ as the driving. The amplitude and phase delay of the response depend on the driving period. For the larger modulation periods $T, u_{\theta}$ responds nearly in phase with the same amplitude as the driving. For smaller modulation periods, the response amplitude decreases and a phase delay is observed, just as in prior studies $10,63,65,66,69$.

A different representation of a modulation cycle is depicted in figure 2.3. Here we plot the data from figure 2.2 parametrically as a function of $\Phi$. A fully quasistationary cycle completely follows the grey line, in which $u_{\theta} /\left\langle u_{\theta}\right\rangle_{t}=u_{i} /\left\langle u_{i}\right\rangle_{t}$. The Wo $=15$ measurement is close to this line. The deviation from this line, which indicates a phase delay, increases for smaller modulation periods.

To study whether the flow responds similarly over the gap width, we extend the analysis from figure 2.2 to the entire radius, see figure 2.4. In the top row, the data is normalized by $\left\langle u_{i}\right\rangle_{t}=2 \pi\left\langle f_{i}\right\rangle_{t} r_{i}=6.3 \mathrm{~m} / \mathrm{s}$, i.e. the same constant for all measurements. The better all lines collapse, the smaller the response amplitude is. For the bottom row, we chose to normalize with $u_{i}(\Phi)=2 \pi r_{i}\left\langle f_{i}\right\rangle_{t}[1+e \sin (\Phi)]$, i.e. the inner cylinder velocity at the corresponding phase in the modulation. Here, when all lines collapse, the modulation is slow enough for the flow to react to the modulation, i.e. the system is in a quasi-stationary state. For comparison, the azimuthal velocity profile for the non-modulated case is shown as a grey line [54]. Figure 2.4(a) and (f) depict the most extreme cases. Furthermore, we show the laminar flow response in the top row. In figure 2.4(a), the azimuthal velocity of the flow is almost constant over a modulation cycle, and therefore $u_{\theta}(r, \Phi)$ is close to the non-modulated statistically stationary solution for $f_{i}=5 \mathrm{~Hz}$; the flow cannot adapt to the quick changes of the inner cylinder. For larger Womersley numbers, the opposite is the case, see figure 2.4 (f). Here, for every phase $\Phi$, the azimuthal velocity profile is identical to the statistically stationary solution for $f_{i}(\Phi)$. This behaviour is surprisingly constant over the entire radius. We note that it might appear as if the correct boundary conditions are not met. However, as shown in [54], the boundary layer at the studied Reynolds number is too thin to resolve from the current measurements.

The laminar flow response is completely different as compared to the measured turbulent case. First, the response in the flow is restricted to a thin layer close to the inner cylinder wall. Calculating the thickness of the Stokes boundary-layer, although slightly off due to the presence of the outer cylinder and a cylindrical coordinate system, gives a similar result, i.e. $\tilde{\delta}\left(\mathrm{Wo}_{\mathrm{O}}=88\right)=0.10, \tilde{\delta}\left(\mathrm{Wo}_{\mathrm{O}}=36\right)=0.24$, and $\tilde{\delta}(\mathrm{Wo}=15)=0.60$ (see table 2.1). Second, the response is radius-dependent, as is 

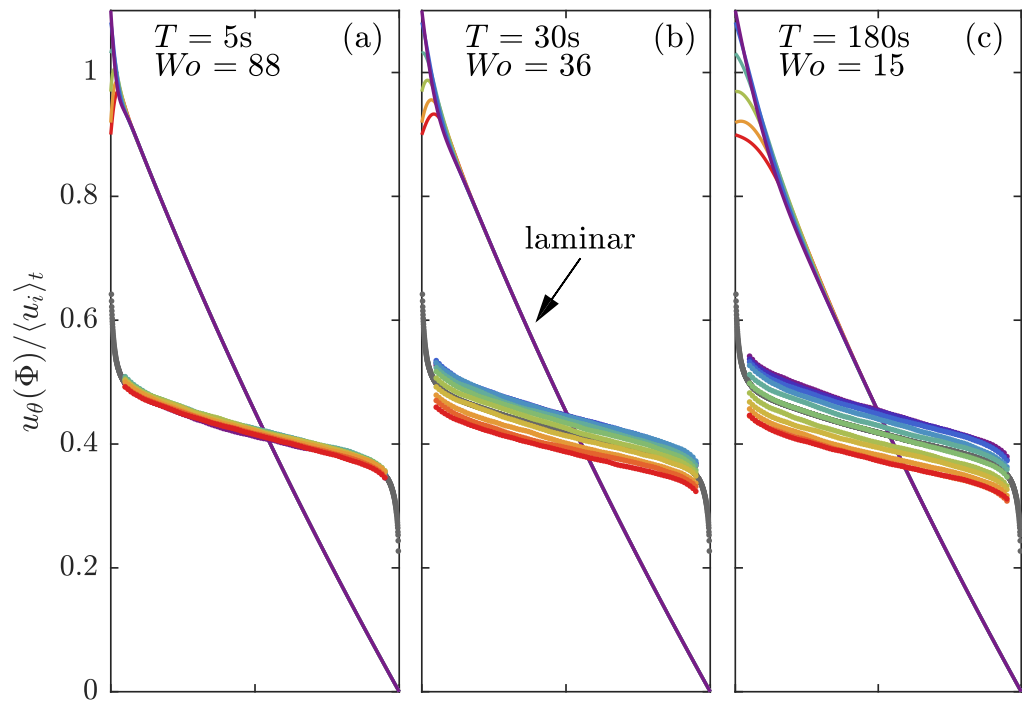

- $\Phi=0.5 \pi$

- $\Phi=0.6 \pi$

$\Phi=0.7 \pi$

- $\Phi=0.8 \pi$

- $\Phi=0.9 \pi$

$\Phi=1.0 \pi$

- $\Phi=1.1 \pi$

$\Phi=1.2 \pi$

$\Phi=1.3 \pi$

- $\Phi=1.4 \pi$

- $\Phi=1.5 \pi$
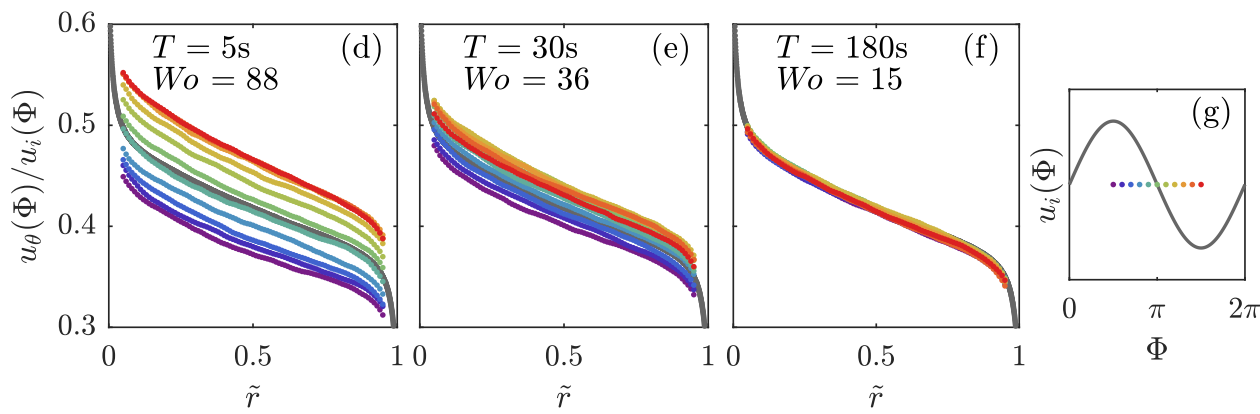

Figure 2.4: Azimuthal velocity profiles as a function of dimensionless radius $\tilde{r}$. All data is phase-averaged and normalized.

Top row (a-c) $u_{\theta}(\Phi)$ is normalized with the time-averaged inner cylinder velocity $\left\langle u_{i}\right\rangle_{t}=6.3 \mathrm{~m} / \mathrm{s}$, i.e. the same constant value for all lines. A collapse of all lines indicates that the response amplitude is small, as is observed for large Wo, see figure (a). Furthermore, we show the response of laminar flow to the modulation, calculated numerically (see method section).

Bottom row (d-f) $u_{\theta}(\Phi)$ is normalized by the instantaneous inner cylinder velocity at phase $\Phi$, i.e. $u_{i}(\Phi)$ (a value between $u_{i}(0.5 \pi)=6.9 \mathrm{~m} / \mathrm{s}$ and $u_{i}(1.5 \pi)=5.7 \mathrm{~m} / \mathrm{s}$ ). A collapse of all lines indicates that the system behaves quasi-stationary, as can be seen for small Wo in figure (f).

The solid grey lines show the azimuthal velocity profile for $\operatorname{Re}_{i}=5 \times 10^{5}$ for the non-modulated, stationary case (data from [54]).

Bottom right (g) The azimuthal velocity $u_{\theta}(\Phi)$ is shown for a series of phases of the modulation; here we show data for phases between $0.5 \pi \leq \Phi \leq 1.5 \pi$, i.e. half of a modulation cycle, as shown in this inset. See also figure 2.2 for the definition of phase $\Phi$. 

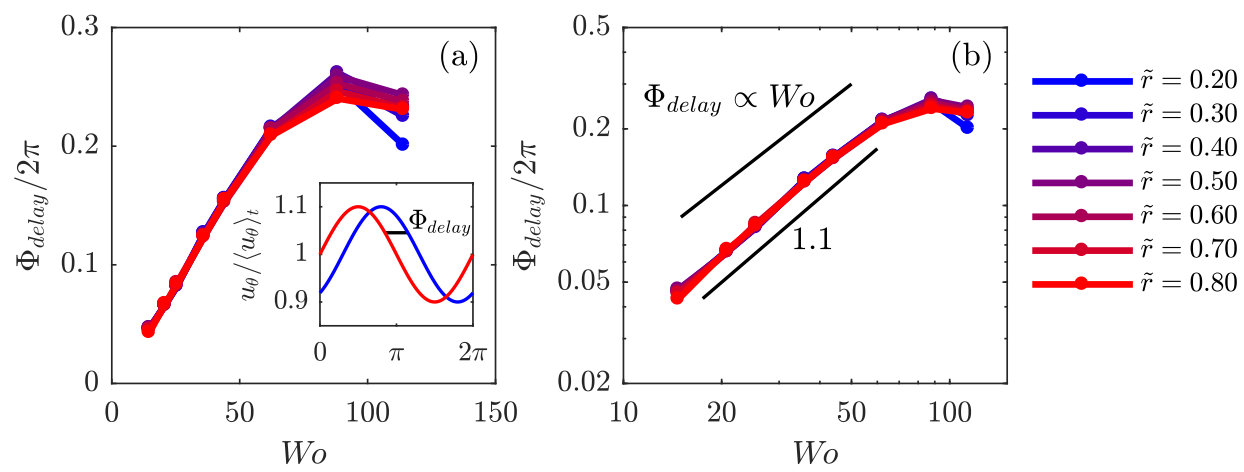

Figure 2.5: The delay between the driving modulation and the fluid velocity response as a function of Womersley number Wo. The delay $\Phi_{\text {delay }}$ is normalized with $2 \pi$ of the modulation. The phase delay is calculated for a number of radii, not showing much difference. The figures show the same data in linear scale (a) and logarithmic scale (b). The results are radially binned within $\tilde{r} \pm 0.025$. The inset in figure a) shows how the phase delay $\Phi_{\text {delay }}$ is defined. $\Phi_{\text {delay }}$ is calculated by cross-correlating both signals. We included the scaling of the response for laminar flow, which equals $\Phi_{\text {delay }} \propto$ Wo.

also known from Stokes oscillating plate theory, as the response decays exponentially with increasing distance from the oscillating wall. These observations highlight how turbulent mixing enhances the transport of the modulation over the entire radius.

\subsubsection{Phase delay}

Up to now the conclusions drawn from figures 2.2 2.3 and 2.4 were only qualitative. Here, we quantify the phase shift and amplitude response for the turbulent case. We extract the phase delay $\Phi_{\text {delay }}$ between the modulation and the response by cross-correlating $u_{i}(t)$ and $u_{\theta}(t)$. We detect the first peak in $u_{i} \star u_{\theta}(\tau)$, and obtain the phase delay by fitting a Gaussian function through this peak and its two neighbouring points, to obtain the peak with increased accuracy. As visible in figure 2.5, at large modulation periods, the phase delay is small, as we already qualitatively concluded from figure 2.2. As the Womersley number increases, the bulk flow cannot follow the changing BCs anymore and it responds with an increasing delay. Within this approximation, 63 calculated, and 10 experimentally found, that the phase delay has a linear dependence on the modulation frequency, i.e. $\Phi_{\text {delay }} \propto \mathrm{Wo}^{2}$. We do not observe a similar behaviour, however. The results in the aforementioned studies, which both study homogeneous and isotropic turbulence (HIT), are significantly different than what we observe in our Taylor-Couette setup, which cannot be regarded as HIT [109].

As visible in figure $2.5 \mathrm{p}$, in these experiments the dependence of $\Phi_{\text {delay }}$ is better described by an effective power law over a range of larger values of Wo, with $\Phi_{\text {delay }} \propto$ $\mathrm{Wo}^{1.1}$. For the laminar case, the phase lag in the Stokes boundary layer problem is 

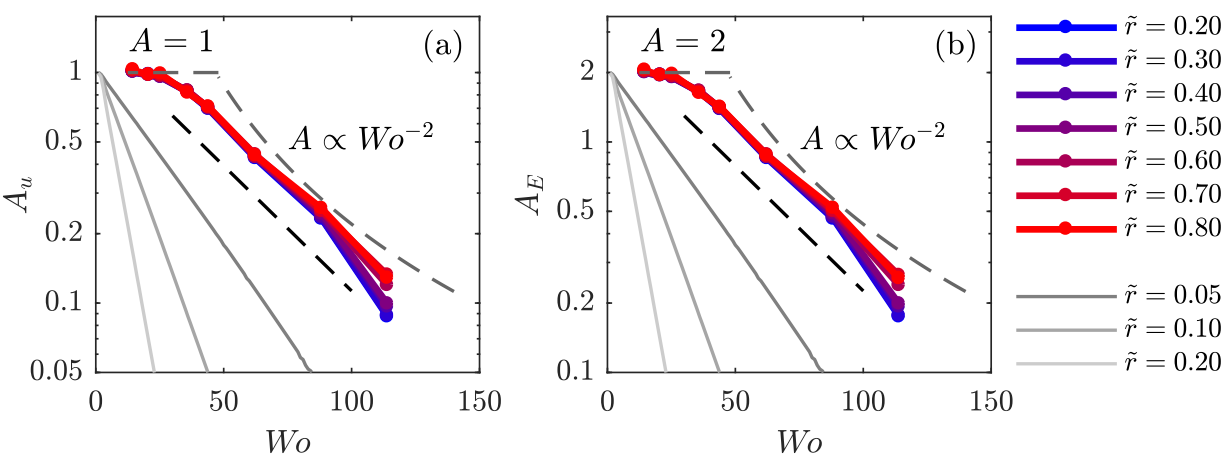

Figure 2.6: Amplitude response as a function of the Womersley number Wo for various dimensionless radii. The coloured lines represent our measurements, and the solid grey lines are numerically calculated laminar flow responses. (a) The response amplitude of the velocity $A_{u}$ and (b) the response amplitude of the energy $A_{E}$. The experimental results are radially binned between $\tilde{r} \pm 0.025$. The dashed grey lines show the scalings of $A$ as predicted by 63 . We included the laminar responses, shown in solid grey lines. A number of radii are included, to highlight the dependence on the radius, which does not exist in the well-mixed turbulent case. The effective slope of the measurements $A \propto e^{-0.025 \text { Wo }}$ is shown in dashed black. This would correspond to the slope of the laminar flow response at $\tilde{r} \approx 0.035$.

calculated as $\Phi_{\text {delay }}=\sqrt{2} \tilde{r}$ Wo. The exponent 1.1 is close to the value of the laminar flow response, in which there is a linear dependance between the Womersley number and the phase delay. The phase lag saturates at around $\Phi_{\text {delay }}=\pi / 2$, similar to what is known in pulsating pipe flow 71,72 and in e.g. periodically forced harmonic oscillators.

We now come to the spatial dependence of the response. Intuitively, one expects an increasing phase delay further away from the modulated wall. Surprisingly, this is not the case. Apparently, the turbulent mixing of this highly turbulent flow prevents the system from having a range of phase delays over the radius, given the fact that the modulation has been "passed on" from the boundary layer to the bulk flow. This can be explained by calculating a characteristic timescale $\tau_{b u l k}$ for the movement from the inner to the outer cylinder, using the Reynolds wind number $\operatorname{Re}_{w}=\sigma\left(u_{r}\right) d / \nu$, in which $\sigma\left(u_{r}\right)$ is the standard deviation of the radial velocity. We estimate $\tau_{b u l k}=$ $d / \sigma\left(u_{r}\right)=d^{2} / R e_{w} \nu . R e_{w}$ for the corresponding $\left\langle R e_{i}\right\rangle_{t}=5 \times 10^{5}$ is known from [219], resulting in a $\tau_{b u l k}=0.27 \mathrm{~s}$. As long as $\tau_{\text {bulk }} \ll T$, the radial dependence of the phase delay and amplitude should be negligible, in agreement with our observations. Such small periods $T$ are unfortunately not accessible experimentally due to the moment of inertia of the cylinders. 


\subsubsection{Amplitude response}

We calculate the amplitude $A$ of the response for both the velocity and kinetic energy, which is defined as $E=\frac{1}{2} \vec{u} \cdot \vec{u} \approx \frac{1}{2} u_{\theta}^{2}$. Following the approach of 63 , the local oscillating response of the velocity and energy is calculated as

$$
\begin{aligned}
\Delta_{u}(t) & =\frac{u_{\theta}(t)}{\left\langle u_{\theta}\right\rangle_{t}}-1, \text { and } \\
\Delta_{E}(t) & =\frac{E(t)}{\langle E\rangle_{t}}-1 .
\end{aligned}
$$

We average $\Delta_{u}(t)$ and $\Delta_{E}(t)$ radially and azimuthally, and make the ansatz that $\Delta_{u, E}(t)$ can be described as:

$$
\Delta_{f i t}(t)=e A(T) \sin \left(2 \pi t / T+\Phi_{\text {delay }}\right) .
$$

$\Delta_{f i t}(t)$ is fitted to $\Delta(t)$ with $A(T)$ as sole fitting parameter. $\Phi_{\text {delay }}$ is not a fitting parameter, as it is calculated using cross-correlation, see figure 2.5. In the case of slow, quasi-stationairy modulations, the amplitude response of the azimuthal velocity can be calculated from equations 2.4 , namely $A_{u}=\left(\frac{(1+e)}{1}-1\right) / e=1$. Strictly speaking is it impossible to describe the kinetic energy with a sinusoidal function, as it has a squared dependence on the velocity, but, as $e$ is small a sine wave can be used within the assumption of a linear response. However, the calculation of $A_{E}$ in the quasi-stationary case is less straight-forward, as the response amplitude varies over the sine wave. We calculate $A_{E}^{\max }=\left((1+e)^{2}-1\right) / e=2.1$ and $A_{E}^{\min }=\left((1-e)^{2}-1\right) /-e=1.9$ as the two extremes, leading to a phase-averaged value of $A_{E}=2.0$. Both response amplitudes will vanish in the limit of infinitely fast modulations, i.e. Wo $\rightarrow \infty$ implies that $A_{u, E} \rightarrow 0$.

As figure 2.6 clearly shows, the fluid completely follows the imposed modulation at larger modulation periods, i.e. amplitude responses of $A_{u}=1$ and $A_{E}=2$ are observed, which corresponds to our expectations. For smaller modulation periods, the response amplitude decreases. We do not observe clean power laws, as predicted assuming HIT by 63 and $[10]$ shown as dashed lines. The response of the flow can better be described by an exponential function, as indicated by the solid black line. This is in line with the laminar flow response, in which the amplitude of the response also is an exponential function of the Womersley number and the distance to the modulated wall. Note that, in contrast to the turbulent case, the amplitude response of the laminar case depends on the radius.

Similar to the phase delay between modulation and response, also in the response amplitude we do not observe any trend over the radius. Here, one could expect a decreasing $A$ at higher radii, i.e. further away modulated wall. Because of the no-slip condition at the wall, the values of $A$ and $\Phi_{\text {delay }}$ directly at the wall are fixed, i.e. $A_{u}\left(r_{i}\right)=1$ and $\Phi_{\text {delay }}\left(r_{i}\right)=0$. At the outer cylinder, $A_{u}\left(r_{o}\right)=0$, hence $\Phi_{\text {delay }}\left(r_{o}\right)$ cannot be defined. Clearly, the boundary layers play a pivotal role in transferring perturbations and modulations to the bulk of the flow. 


\subsection{Summary and conclusions}

To conclude, we studied periodically driven Taylor-Couette turbulence. We drove the inner cylinder sinusoidally, and measured the local velocity using PIV. Consistent with earlier studies and theoretical expectations, we observe a phase delay and declining velocity response as we increase the Womersley number. Most surprisingly, we did not observe a radial dependence of the phase delay in the bulk of the flow, nor of the amplitude response, in contrast to the expectation one might have that there could be a larger influence of the modulation on the flow close to the modulated wall. Apparently, a radial dependence of $A$ and $\Phi_{\text {delay }}$ is prevented by the strong mixing in this turbulent flow. Even though we did not measure directly in the boundary layers, their vital importance in transferring modulations to the bulk flow is evident. This contrasts our numerical results for laminar flow, where a strong radial dependence is observed, and the response of the flow is confined to a thin layer close to the modulated wall. Therefore it is even more remarkable that the scaling relations of both the phase delay and the amplitude response are similar to what had been found for laminar flows.

To further study this interesting phenomenon, direct numerical simulations are necessary to cover the extremely high Womersley number range, which is inaccessible in experiments. Using such data, it will be possible to study the interplay between the modulated cylinder, the boundary layers and the bulk in more detail, as the entire flowfield will then be available. Another domain of "terra incognita" is the study of modulations with larger amplitude. Here, we limited ourselves to a modulation amplitude of $e=0.1$. Larger values induce non-linear effects, and linear response type assumptions such as those made in equations $(2.4)$ and 2.5 will then not be valid anymore. 


\section{Part II}

\section{Roughness in turbulence}




\section{Chapter 3}

\section{Wall roughness induces asymptotic ultimate turbulence ${ }^{T}$}

Turbulence governs the transport of heat, mass, and momentum on multiple scales. In real-world applications, wall-bounded turbulence typically involves surfaces that are rough; however, characterizing and understanding the effects of wall roughness for turbulence remains a challenge. Here, by combining extensive experiments and numerical simulations, we examine the paradigmatic Taylor-Couette system, which describes the closed flow between two independently rotating coaxial cylinders. We show how wall roughness greatly enhances the overall transport properties and the corresponding scaling exponents associated with wall-bounded turbulence. We reveal that if only one of the walls is rough, the bulk velocity is slaved to the rough side, due to the much stronger coupling to that wall by the detaching flow structures. If both walls are rough, the viscosity dependence is thoroughly eliminated, giving rise to asymptotic ultimate turbulence - the upper limit of transport - the existence of which was predicted more than fifty years ago. In this limit, the scaling laws can be extrapolated to arbitrarily large Reynolds numbers.

\footnotetext{
${ }^{1}$ Published as: Xiaojue Zhu*, Ruben A. Verschoof*, Dennis Bakhuis, Sander G. Huisman, Roberto Verzicco, Chao Sun, and Detlef Lohse, Wall roughness induces asymptotic ultimate turbulence, Nature Physics 14, 417-423 (2018).

Zhu and Verschoof contributed equally to this work. Simulations by Zhu. Experiments by Verschoof and Bakhuis. Data analysis by Zhu and Verschoof. Zhu, Verschoof and Lohse wrote the paper. Verzicco, Sun and Lohse supervised the project. All authors discussed the results and proofread the paper.
} 


\subsection{Introduction}

While the vast majority of studies on wall-bounded turbulence assumes smooth walls, in engineering applications and even more so in nature, flow boundaries are in general rough, leading to a coupling of the small roughness scale to the much larger outer length scale of the turbulent flow. This holds for the atmospheric boundary layer over canopy or buildings, for geophysical flows, in oceanography, but also for many industrial flows such as pipe flow, for which the presumably most famous (though controversial) study on roughness was performed [145]. For more recent works on the effect of wall roughness in (pipe or channel) turbulence we refer to various studies 146 149], reviews [150,151], or textbooks 13, 152].

Rather than the open channel or pipe flow, here we use a Taylor-Couette (TC) facility [51, which is a closed system obeying global balances and at the same time allows for both accurate global and local measurements. The overall torque $\tau$ in TC flow to keep the cylinders at constant angular velocity, is connected with the spatially and time averaged energy dissipation rate $\epsilon_{u}$. This can be expressed in terms of the friction factor $13,51,152$

$$
C_{f}=\frac{\tau}{\ell \rho_{f} \nu^{2}\left(\operatorname{Re}_{i}-\eta \operatorname{Re}_{o}\right)^{2}}=\frac{\pi \eta}{(1-\eta)} \frac{\epsilon_{u}}{\left(U_{i}-\eta U_{o}\right)^{3} /\left(r_{i}+r_{o}\right)} .
$$

Here $U_{i, o}$ are the velocities of the inner resp. outer cylinder, $r_{i, o}$ their radii, $\nu$ the kinematic viscosity (together defining the inner and outer Reynolds numbers $\operatorname{Re}_{i, o}=$ $\left.U_{i, o} d / \nu\right), \rho_{f}$ the density of the fluid, $\ell$ the height of the TC cell, $d=r_{o}-r_{i}$ the gap width, and $\eta=r_{i} / r_{o}$ the ratio between outer and inner cylinder radius. The key question now is: how does the friction factor $C_{f}$ depend on the (driving) Reynolds number $\mathrm{Re}_{i, o}$ and how does wall roughness affect this relation?

Alternatively, the Reynolds number dependence of the friction factor $C_{f}$ can be expressed as a "Nusselt number" $\mathrm{Nu}_{\omega}=\tau /\left(2 \pi \ell \rho_{f} J_{\text {lam }}^{\omega}\right)$ (i.e. the dimensionless angular velocity flux normalized with the laminar flux $J_{\text {lam }}^{\omega}=2 \nu r_{i}^{2} r_{o}^{2}\left(\omega_{i}-\omega_{o}\right) /\left(r_{o}^{2}-r_{i}^{2}\right)$ [189]) depending on the Taylor number $\mathrm{Ta}=\frac{1}{64} \frac{(1+\eta)^{4}}{\eta^{2}} d^{2}\left(r_{i}+r_{o}\right)^{2}\left(\omega_{i}-\omega_{o}\right)^{2} \nu^{-2}$ 51, with $\omega_{i, o}$ the angular velocity of the inner resp. outer cylinder. This notation $\mathrm{Nu}_{\omega}(\mathrm{Ta})$ stresses the analogy between TC flow and Rayleigh-Bénard flow (RB) 47, 48], the flow in a box heated from below and cooled from above, where the Nusselt number $\mathrm{Nu}$ (the dimensionless heat flux) depends on the Rayleigh number Ra (the dimensionless temperature difference). For that system in 1962 Kraichnan 185 had postulated a so-called "ultimate scaling regime" [5, 8, 51, 153, 154, 219]

$$
\mathrm{Nu} \propto \mathrm{Ra}^{1 / 2}(\log \mathrm{Ra})^{-3 / 2}
$$

(for fixed Prandtl number). In analogy, such an ultimate regime also exists for TC flow, namely

$$
\mathrm{Nu}_{\omega} \propto \mathrm{Ta}^{1 / 2}(\log \mathrm{Ta})^{-3 / 2}
$$


as worked out in Ref. 186]. In fact, in that reference slightly different log-dependences were derived, namely

$$
\begin{aligned}
\mathrm{Nu} & \propto \mathrm{Ra}^{1 / 2} \mathcal{L}(\mathrm{Re}), \quad \text { and } \\
\mathrm{Nu}_{\omega} & \propto \mathrm{Ta}^{1 / 2} \mathcal{L}(\mathrm{Re}),
\end{aligned}
$$

where $\mathcal{L}(\operatorname{Re}(\mathrm{Ra}))$ resp. $\mathcal{L}(\operatorname{Re}(\mathrm{Ta}))$ are logarithmic dependences (see Methods and also Ref. [186]). Irrespective of whether one takes the logarithmic dependences (3.2) resp. (3.3) or (3.4) resp. (3.5), for smooth walls due to these log-corrections the effective scaling exponent for the largest experimentally achievable Rayleigh (Taylor) numbers is only around 0.38 and not $1 / 2$, i.e., $\mathrm{Nu} \propto \mathrm{Ra}^{0.38}$ resp. $\mathrm{Nu}_{\omega} \propto \mathrm{Ta}^{0.38}$. This effective exponent 0.38 has indeed been observed in large Ra RB experiments 153, 154, large Ta TC experiments [51,219] and numerical simulations 5, 51. The log-corrections, which are intimately connected with the logarithmic boundary layers [155], thus prevent the observation of the asymptotic ultimate regime exponent $1 / 2$, which is the exponent of mathematically strict upper bounds for RB and TC turbulence 156 158]. Historically, whether such asymptotic $1 / 2$ scaling exists or not has triggered enormous debate, see e.g. [47. In the last two decades, great efforts have been put into reaching this regime with smooth boundaries, both experimentally and numerically. Today, this issue is often considered as one of the most important open problems in the thermal convection community. In fact, the exponent $1 / 2$ has only been achieved with rough walls [159 as presumably a transient, local effective scaling, which saturates back to smaller exponent at even larger $\mathrm{Ra}$ [47,160 161], or in artificial configurations, such as numerical simulations of so-called "homogeneous convective turbulence" 162 with periodic boundary conditions and no boundary layers, or experimental realisations thereof such as in Refs. 163 164.

The asymptotic exponent $1 / 2$ in the $\mathrm{Nu}$ vs. Ra resp. $\mathrm{Nu}_{\omega}$ vs. Ta scaling laws corresponds to a friction factor $C_{f}$ being independent of the Reynolds number. Vice versa, expressed in terms of the friction factor, Eqs. 3.2 to (3.5) can be written with a logarithmic dependence, analogous to the so-called Prandtl-von Kármán skin friction law 13, 152, 165 for pipe flow, i.e.

$$
1 / \sqrt{C}_{f}=a \log _{10}\left(\operatorname{Re}_{i} \sqrt{C}_{f}\right)+b,
$$

which can be obtained by assuming that the boundary layer profiles at each cylinder wall are logarithmic and match at the middle gap 6, 54,55]. Here $a$ and $b$ are fitting constants connected with the von Kármán constant $\kappa$.

How to get rid of the log-correction and to thus achieve asymptotic ultimate turbulence with a $1 / 2$ power law or equivalently a Reynolds number independent friction factor for TC flow? The path we will follow here is to introduce wall roughness 125, 166 170]. By combining direct numerical simulations (DNS) and experiments (EXP), we explore five decades of Ta to present conclusive evidence that the $1 / 2$ power law can be realized, thus achieving the asymptotic ultimate regime. Moreover, we will give a theoretical justification for the findings based on measurements of the global and local flow structures and extend the analysis also to outer cylinder rotation. 

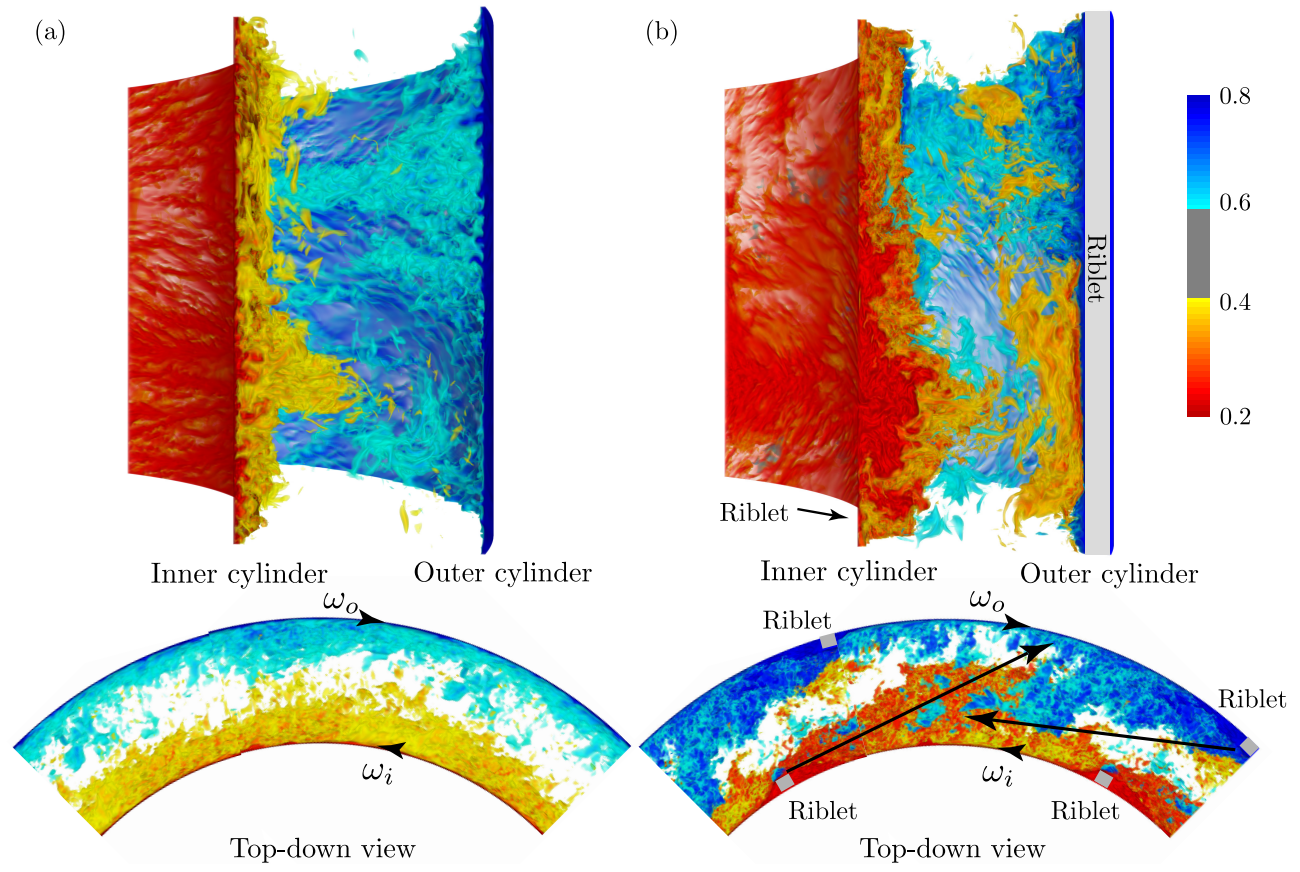

Figure 3.1: Plume structures for smooth and rough Taylor-Couette turbulence, i.e. turbulent flow between two co-axial rotating cylinders, with inner cylinder rotates at angular velocity $\omega_{i}$ and outer cylinder at $\omega_{o}$. Here the volume renderings of azimuthal velocity at $\mathrm{Ta}=2.15 \times 10^{9}$ and Rossby number $\mathrm{Ro}^{-1}=-0.2$ are shown, from numerical simulations (see Methods for more details). a, Both cylinders are smooth. The plumes are generated on both cylinders and form the structure of Taylor rolls and they are concentrated in local regions and can not reach the other cylinder. b, Both cylinders are rough with 6 ribs of height $h=0.1 d$. Even in the rough case, Taylor rolls still exist. Now the plumes are also generated on top of the roughness elements and shed to the opposing cylinder. The arrows in the top-down views illustrate the directions of plumes shedding. All plots share the same colormap, based on the value of the local angular velocity. 
Four cases will be considered: SS, SR, RS, and RR, where the first (second) letter specifies the configuration of the inner (outer) cylinder, which can be either rough $(\mathrm{R})$ or smooth $(\mathrm{S})$. In both DNS and EXP, the radius ratio between the two cylinders is $\eta=0.716$. The cylinders were made rough by attaching 1 to 192 vertical ribs with identical heights ranging from $1.5 \%$ to $10 \%$ of the gap width $d$ and a square cross-section over the entire TC cell on none, both, or either one of the cylinders (see Methods section). To give the reader an impression of the flow organization, typical flow structures of a smooth case and a rough case are shown in Fig. 3.1.

\subsection{Global scaling relations}

In this section we address the question of how roughness modifies the global scaling relations. First, we focus on the cases of 6 ribs with identical heights $h=0.075 d$. The global dimensionless torques, $\mathrm{Nu}_{\omega} \propto \mathrm{Ta}^{\gamma}$, for the four cases, with increasing $\mathrm{Ta}$ and fixed outer cylinder, are shown in Fig. 3.2 a. Combining EXPs and DNSs, the range of Taylor number studied here extends over five decades. Similarly to what was shown in various recent studies [4,5,51,55, 154, 171], for the SS case, an effective scaling of $\mathrm{Nu}_{\omega} \propto \mathrm{Ta}^{0.38 \pm 0.02}$ is observed in the DNS, corresponding to the ultimate regime with logarithmic corrections 185,186 . A very similar scaling exponent $\mathrm{Nu}_{\omega} \propto \mathrm{Ta}^{0.39 \pm 0.01}$ is found in EXPs, demonstrating the excellent agreement between DNS and EXPs.

Dramatic enhancements of the torques are clearly observed with the introduction of wall roughness, resulting in the transition of $\mathrm{Nu}_{\omega}$ from $\mathcal{O}\left(10^{2}\right)$ to $\mathcal{O}\left(10^{3}\right)$. Specifically, when only a single cylinder is rough, the logarithmic corrections reduce and the scaling exponents marginally increase, implying that the scaling is dominated by the single smooth wall. For the $\mathrm{RR}$ case, the best power law fits give $\mathrm{Nu}_{\omega} \propto \mathrm{Ta}^{0.50 \pm 0.02}$, both for the numerical and experimental data, suggesting that the logarithmic corrections are thoroughly canceled. This state with the scaling exponent $1 / 2$ corresponds to the asymptotic ultimate turbulence predicted by Kraichnan 185. The compensated plots of insets of $\mathrm{Nu}_{\omega} / \mathrm{Ta}^{\gamma}$ show the robustness and the quality of the scalings.

When expressing the relation between the global transport properties and the driving force in terms of the Reynolds number dependence of the friction factor $C_{f}$, we obtain Fig. 3.2 b. For the SS case, the fitting parameters $a$ and $b$ yield a von Kármán constant $\kappa=0.44 \pm 0.01$, which is slightly larger than the standard value of 0.41 due to the curvature effect $[54,155,172$. This agrees well with the previous measurements on TC with smooth walls [53]. For the RR case, for large enough driving the friction factor $C_{f}$ is found to be independent of $\mathrm{Re}_{i}$, but dependent on roughness height, namely $C_{f}=0.21$ in the DNS and $C_{f}=0.23$ in the EXP for roughness height $h=0.075 d$. The results here are consistent with the asymptotic ultimate regime scaling $1 / 2$ for $\mathrm{Nu}_{\omega}$ and indicate that the Prandtl-von Kármán log-law of the wall [13 152 with wall roughness can be independent of $\operatorname{Re}_{i} 13,145,150$ 152, which has been verified recently for Taylor-Couette flow 173 . For the RS and SR cases, one boundary is rough and the other is smooth such that the friction law lies in between $\mathrm{RR}$ and SS lines. 
We further show the RR case with ribs of different heights, ranging from $1.5 \%$ to $10 \%$ of the gap width $d$ in Fig. 3.2 , displaying its similarity with the Nikuradse 145 and Moody 84 diagrams for pipe flow. It can be seen that once $h \geqslant 0.05 d$ and $\operatorname{Re}_{i} \geqslant 8.1 \times 10^{3}\left(\mathrm{Ta} \geqslant 10^{8}\right)$, the asymptotic ultimate regime can always be achieved in both DNS and EXP.

Analogously, we note that in pipe flow, the same phenomenon of Reynolds number independent friction factor with wall roughness was observed in the fully rough regime 13, 145,150 152, where the characteristic heights of the roughness elements in wall units $h^{+}>70$ 13. 152. In contrast, for $\mathrm{Ta}=10^{8}$, for the roughness height $h / d=0.05$, $h / d=0.075$, and $h / d=0.10, h^{+}=51, h^{+}=61$, and $h^{+}=71$, respectively. Indeed, almost all of our data are in the fully rough regime for cases with $h \geqslant 0.05 d$ and $\mathrm{Ta} \geqslant 10^{8}$, thus corroborating the current conclusion that adopting wall roughness is one way to achieve asymptotic ultimate turbulence in TC.

We now interpret the asymptotic ultimate torque scalings through an extension of the Grossmann-Lohse (GL) theory [186], by accounting for the Prandtl-von Kármán log-law of the wall 152 in the presence of roughness. To demonstrate this extension, for simplicity we take as example the case of only inner cylinder rotation. For a smooth wall, the energy dissipation rate in the $\log$ region scales with $\epsilon_{u} d^{4} / \nu^{3} \propto$ $\operatorname{Re}_{i}^{3}\left(u_{\tau} / U\right)^{3} \ln \left(\operatorname{Re}_{i} u_{\tau} / U\right)$ 186, which stems from the integration of the Prandtl-von Kármán log-law of the wall, where $u_{\tau}$ is the friction velocity and $U$ the velocity of the inner cylinder. The log term in the law is dependent on $\operatorname{Re}_{i}$, which is the origin of the logarithmic correction term $\mathcal{L}(\mathrm{Re})=\left(u_{\tau} / U\right)^{3} \ln \left(\operatorname{Re}_{i} u_{\tau} / U\right)$ and thus for the deviation from the asymptotic ultimate regime scaling $\epsilon_{u} d^{4} / \nu^{3} \propto \operatorname{Re}_{i}^{3}$, leading to a decrease of the effective scaling exponent. However, with roughness, as stated before, the log term in the law of the wall becomes independent of $\operatorname{Re}_{i}, 13,150,152,173$, which correspondingly renders this correction constant. Translating this argument for the energy dissipation rate $\epsilon_{u}\left(\mathrm{Re}_{\mathrm{i}}\right)$ back to the dimensionless torque $\mathrm{Nu}_{\omega}$ and the driving force $\mathrm{Ta} 189$, we obtain $\mathrm{Nu}_{\omega} \propto \mathrm{Ta}^{1 / 2}$, i.e. the effect of the logarithmic term on the scaling vanishes; see Methods for details.

One distinct difference between TC and pipe flow is that in a TC system, the inner and outer cylinders can rotate independently, resulting in a second control parameter, namely the rotation ratio $a=-\omega_{o} / \omega_{i}$ of the two cylinders. Just as for smooth walls [4,51, also for rough walls the $\mathrm{Nu}_{\omega} \propto \mathrm{Ta}^{\gamma}$ scaling exponents are independent of the rotation ratio $a$ in the studied rotation ratio regime; see Supplementary Fig. S2. As known since Taylor [174], the inner cylinder rotation has a destabilizing effect on the flow, whereas outer cylinder rotation has a stabilizing effect. For TC flow with smooth walls, it was found that the optimal transport rotation ratio $a_{\text {opt }}$ between the two cylinders, where the torque reaches the maximum for a specific driving Ta, is around $a_{o p t}=0.36$ [52,175], and not zero, as one may have assumed. This is attributed to the existence of the strong Taylor rolls between the counter-rotating cylinders when $a \approx a_{\text {opt }}$. Only for strong enough counter-rotation $\left(a>a_{\text {opt }}\right)$ does the stabilization through the counter-rotating outer cylinder take over [176]. Here, we address the question whether this optimal transport rotation ratio shifts or stays the 

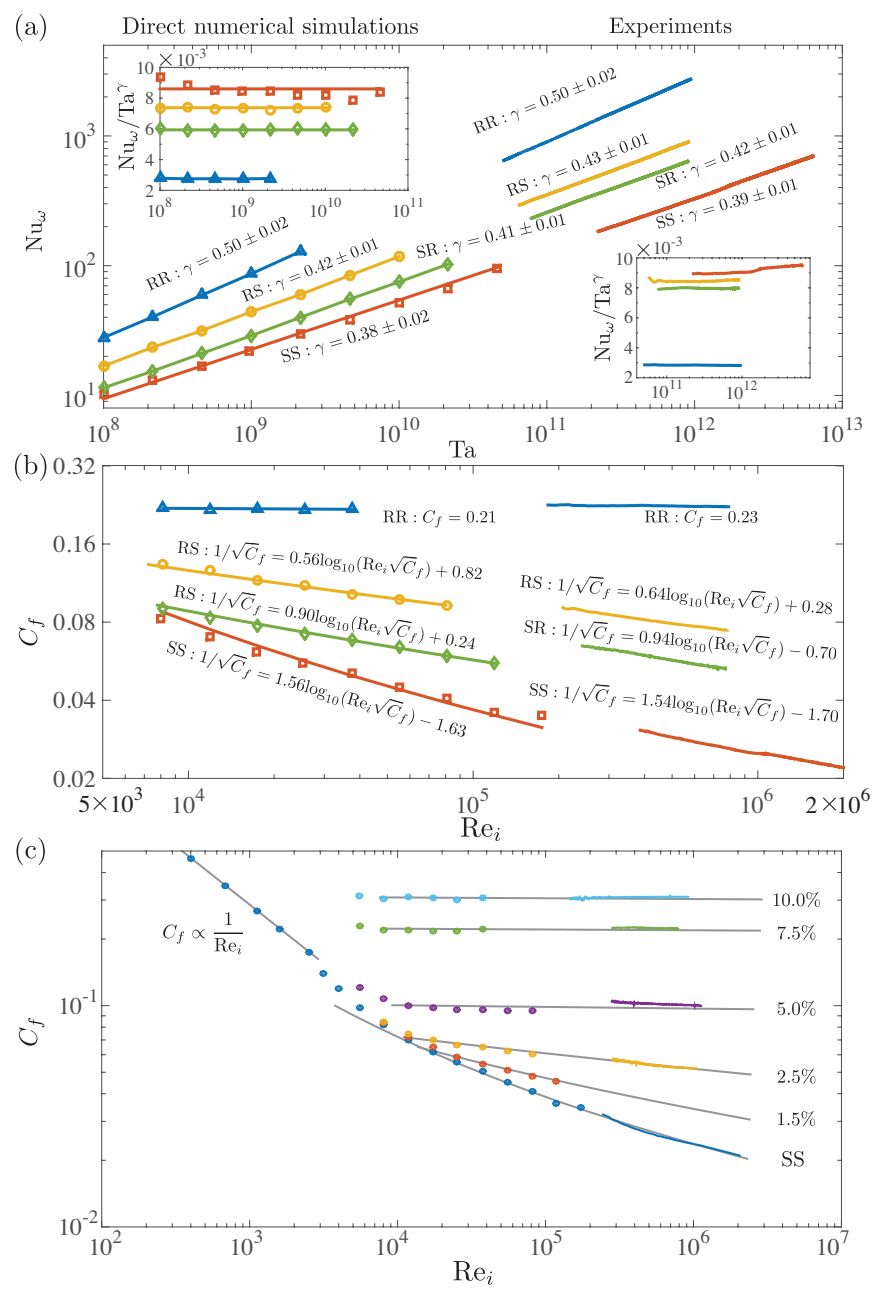

Figure 3.2: Global torque and friction factor scalings in both DNS (symbols), and experiments (colored lines). a, The dimensionless torque as a function of Taylor number Ta. Four cases are shown: (SS) both cylinders smooth; (SR) smooth inner, rough outer; (RS) rough inner, smooth outer; and (RR) both cylinders rough, with the exponent $\gamma$ in the power law relation $\mathrm{Nu}_{\omega} \propto \mathrm{Ta}^{\gamma}$ shown for every case. The insets depict the compensated plots $\mathrm{Nu}_{\omega} / \mathrm{Ta}^{\gamma}$, showing the quality of the scaling. b, The friction factor $C_{f}$ as a function of the inner cylinder Reynolds number $\mathrm{Re}_{i}$. The lines show the best fits of the Prandtl friction law $1 / \sqrt{C}_{f}=a \log _{10}\left(\operatorname{Re}_{i} \sqrt{C}_{f}\right)+b$, with all prefactors shown in the figures. For $\mathbf{a}$ and $\mathbf{b}, 6$ ribs were used and the roughness height is $h=0.075 d$. For the $\mathrm{RR}$ case, $\mathrm{Re}_{i}$ independent friction factors are revealed. c, The friction factor $C_{f}$ for RR cases with 6 ribs of different heights, ranging from $1.5 \%$ to $10 \%$ of the gap width $d$. 
same in the presence of roughness. The results are shown in Fig. 3.3 . We find that when either one of the cylinders is rough, the effect of that rough cylinder is enhanced in several ways, as we will now elaborate.

In the SR case, $a_{\text {opt }, S R}^{D N S}=0.09 \pm 0.03$ and $a_{\text {opt }, S R}^{E X P}=0.11$, i.e. little outer cylinder rotation is necessary to reduce the angular velocity transport with the help of the roughness elements on it, which are thus not so effective. In contrast, a rough inner cylinder is much more effective to enhance the momentum transport. The optimal transport peak for the RS case occurs at much larger rotation ratio, $a_{\text {opt }, R S}^{D N S}=0.69 \pm$ 0.05 and $a_{o p t, R S}^{E X P}=0.84$, as very strong outer cylinder rotation is needed to suppress turbulence originating from the rough inner cylinder. In this case the stabilizing effect of the smooth outer cylinder becomes inefficient.

Finally, in the RR case, the effects of the inner cylinder and outer cylinder are balanced in a similar way as in the SS case, resulting in similar values of $a_{\text {opt }, R R}^{D N S}=$ $0.28 \pm 0.03$ and $a_{o p t, R R}^{E X P}=0.31$ as found in the SS case $\left(a_{o p t, S S}^{D N S}=0.30 \pm 0.03\right.$ and $\left.a_{o p t, S S}^{E X P}=0.34\right)$. At optimal rotation ratio $a_{\text {opt }}$, the enhanced shear is caused by Taylor rolls 5, 52, 177, 178]. This indicates that even in the presence of roughness, Taylor rolls still exist, as visible in Fig. 3.1p. We further notice that the optimal transport properties are dependent on the roughness height, as shown in Supplementary Fig. S3. As expected, when the roughness height is smaller, $a_{o p t}$ for SR and RS cases are closer to $a_{\text {opt }}$ for the SS case. On the contrary, when the roughness height is larger, $a_{\text {opt }}$ for SR and RS cases deviates more from $a_{\text {opt }}$ for the SS case. This can be clearly seen from Supplementary Fig. S4.
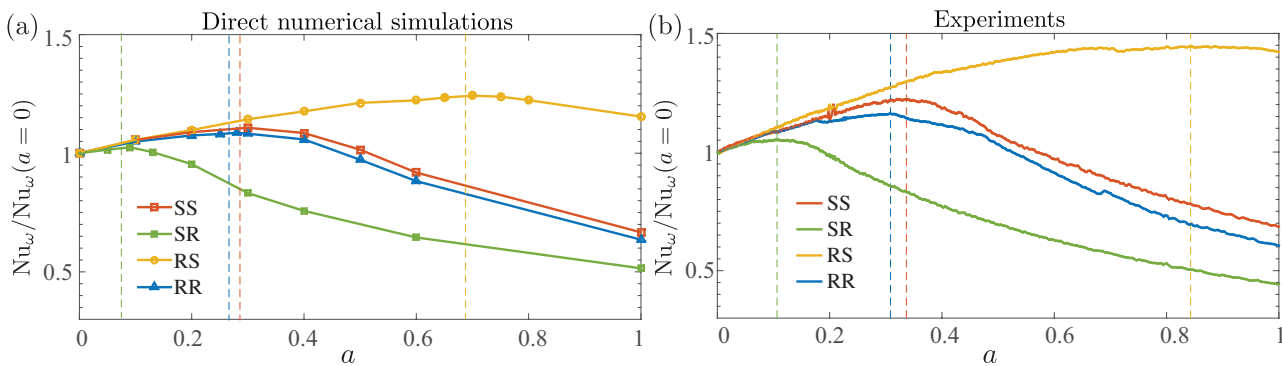

Figure 3.3: Optimal transport peak. $\mathrm{Nu}_{\omega}$ as function of $a$ for constant driving strength, normalized by its value for $a=0$. For both EXP and DNS, 6 ribs were used and the roughness height is $h=0.075 d$. a, DNSs with Ta $=1 \times 10^{9}$. The optimal transport peaks are located at $a_{o p t, S S}^{D N S}=0.30 \pm 0.03, a_{o p t, S R}^{D N S}=0.09 \pm 0.03$, $a_{o p t, R S}^{D N S}=0.69 \pm 0.05$ and $a_{o p t, R R}^{D N S}=0.28 \pm 0.03$. b, Experiments with Ta $=4 \times 10^{11}$. The optimal transport peaks for the four cases are located at $a_{o p t, S S}^{E X P}=0.34, a_{\text {opt }, S R}^{E X P}=$ $0.11, a_{o p t, R S}^{E X P}=0.84$ and $a_{o p t, R R}^{E X P}=0.31$. All optimal transport peaks are indicated by the dashed lines, with the respective colors. 

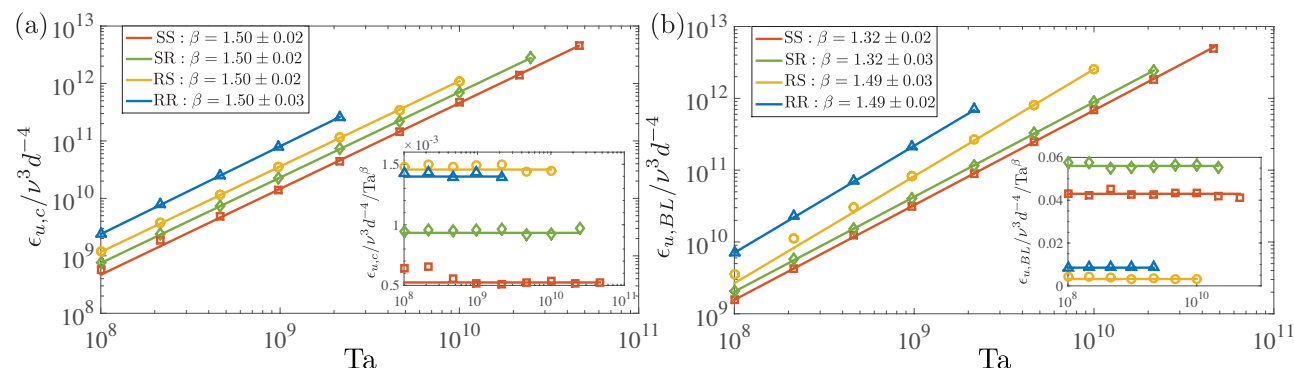

Figure 3.4: Local energy dissipation rate from simulations. Local energy dissipation rate in the bulk $\epsilon_{u, c}$ (at the center of the gap, averaged over the height) and in the inner cylinder boundary layer $\epsilon_{u, B L}$ (averaged in the range from the wall to the distance corresponding to the maximum root mean square of the azimuthal velocity) as a function of Ta, with $\beta$ as the scaling exponent. For the rough cases, 6 ribs were used and the roughness height is $h=0.1 d$. The symbols are the numerical data and the lines show the best fits. a, The bulk energy dissipation rate follows $\epsilon_{u, c} \propto \mathrm{Ta}^{1.50} \propto R e_{i}^{3}$, irrespective of whether the wall is smooth or rough. b, The boundary layer dissipation rate at the inner wall follows $\epsilon_{u, B L} \propto \mathrm{Ta}^{1.32}$ for the cases with smooth walls, while it scales with $\epsilon_{u, B L} \propto \mathrm{Ta}^{1.50}$ for the cases with rough inner wall.

\subsection{Local flow organization and profiles}

Till now, we have focused on the global transport properties. However, the details of the boundary layer-bulk interaction, and in particular how the local scalings of the energy dissipation rates affect the global ones, are still unknown. To verify above sketched theory, from our DNS data we split the mean energy dissipation rate (Eq. 3.1) into boundary layer and bulk contributions, following the GL approach 179,180]. In Fig. 3.4(a), the local energy dissipation rates at mid-gap $\epsilon_{u, c}$ are shown as a function of Ta (only inner cylinder rotation, $a=0$ ). It is clear that no matter whether the wall is smooth or rough, the bulk energy dissipation rate follows $\epsilon_{u, c} \propto \mathrm{Ta}^{3 / 2} \propto \operatorname{Re}_{i}^{3}$, which corresponds to the asymptotic ultimate regime without any logarithmic correction. In analogy, for $\mathrm{RB}$ turbulence, the same scaling exponent $\epsilon_{u, c} \propto \mathrm{Ra}^{3 / 2}$ was reported in Refs. [181, 182. Therefore, the crucial element determining the overall scaling is the dissipation rate in the boundary layer. To further confirm this, in Fig. 3.4(b) we show the local energy dissipation rates of the boundary layer $\epsilon_{u, B L}$ (averaged in the range from the wall to the distance corresponding to the maximum root mean square of the azimuthal velocity). For the case with smooth walls, we find $\epsilon_{u, B L} \propto \mathrm{Ta}^{1.32}$ because of the $\mathrm{Re}_{i}$-dependent velocity profile, while for the boundary layers at rough walls we have $\epsilon_{u, B L} \propto \mathrm{Ta}^{3 / 2}$ because, as shown above, roughness cancels out the $\mathrm{Re}_{i^{-}}$ dependence in $\mathcal{L}\left(\operatorname{Re}_{i}\right)$ and thus restores the asymptotic ultimate regime scaling. The competition between the boundary layer and bulk ultimately determines the global scalings.

We now detail the origin of the enhanced torque. With roughness, the main 
contribution to the torque originates from the pressure differences between the side surfaces of rough elements, rather than from viscous forces $13,150,152,173$. With roughness, we therefore expect the shear rate close to the rough wall to decrease significantly, as compared to the smooth case. This is clearly shown in Fig. 3.5. with smooth cylinders, the normalized velocity profiles are characterized by a bulk region in which the velocity is relatively constant, $U_{\theta}=0.45 r_{i} \omega_{i}$ (whereas for pipe flow, this is not the case, see Supplementary Fig. S5). In case one single cylinder is rough, the bulk velocity is completely dominated by the velocity of the rough cylinder, or in other words, the bulk is enslaved to the rough wall. In the RR case, as there the torque is dominated by pressure forces, the shear rate at the rough cylinder is still smaller as compared to the smooth case. The implication is that with roughness, a larger fraction of energy dissipates in the bulk, and thus the system becomes bulk dominant. As mentioned before, the bulk energy dissipation rate follows $\epsilon_{u, c} \propto \mathrm{Ta}^{3 / 2}$, which implies the asymptotic ultimate regime. The more the bulk dominates the energy dissipation rate, the better the asymptotic ultimate regime manifests itself. This is indeed verified by the flow structure in Fig. 3.1, where for the rough case, the plumes shedding from the roughness elements on one wall elongate towards the other wall and push more energetic fluid elements into the bulk, as compared to the smooth case, leading to more energy dissipation in the bulk.

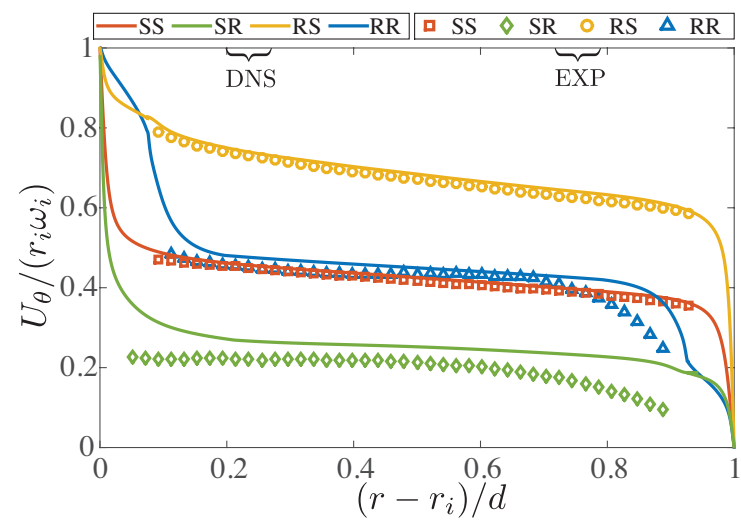

Figure 3.5: Mean velocity profiles. Normalized azimuthal velocity $U_{\theta}(r) /\left(r_{i} \omega_{i}\right)$ profiles as a function of the normalized radius $\left(r-r_{i}\right) / d$ for only inner cylinder rotation. For both EXP and DNS, 6 ribs were used and the roughness height is $h=0.075 d$. Experimental and numerical data are shown in the same figure. EXP: $\mathrm{Re}_{i}=5 \times 10^{5}$ and DNS: $\mathrm{Re}_{i}=3.74 \times 10^{4}$. The experimental results were obtained using PIV.

\subsection{Controlling ultimate turbulence}

To bridge the gap between the effective ultimate scaling exponent 0.38 for the smooth case $5,51,153,154$ and the asymptotic ultimate scaling exponent 0.5 for the RR 
case and thus to actively control ultimate turbulence, we vary the sparseness of the roughness elements while keeping the height of the riblets fixed at $7.5 \%$ of the gap width. To show how this will change the results, as an example, in Fig. 3.6(a), we show the $\mathrm{Nu}_{\omega}$ vs. Ta scaling for the case of 2 ribs (very sparse). The effective scaling exponent $\gamma$ for the RR case is then smaller than 0.5 (i.e. 0.47 ), so the asymptotic ultimate regime is not yet achieved in this situation, in contrast to Fig. 2, when there are six ribs, for which $\gamma=0.5$. We then continuously vary the number of ribs from 1 (very sparse) to 192 (very dense). Correspondingly, the spacing between the rough elements $s / h$ mounted on the inner wall varies from 208.44 to 0.07 . We note that in pipe and BL flows, there is a distinction between $\mathrm{k}$ - and d-types of roughness, and a close spacing will make the roughness behave more like d-type roughness compared with k-type roughness 150,151]. In Fig. 3.6(b), we see that the effective scaling exponent is continuously changing with $s / h$. There is an optimal $s / h=7$ where the effective scaling exponent is the largest, corresponding to k-type roughness. To explain why the effective scaling exponent depends on $s / h$, in Fig. 3.6.(c) we split the global $\mathrm{Nu}_{\omega}$ into two parts, namely the viscous force contribution $\left(\mathrm{Nu}_{v}\right)$ and the pressure force contribution $\left(\mathrm{Nu}_{p}\right)$. Clearly, when the effective scaling exponent is higher, the pressure forces are more dominant.

We propose a simple model which can recover the effective scaling exponent. The model is based on the fact that in the smooth case, only viscous forces contribute to $\mathrm{Nu}_{\omega}$, resulting in $\mathrm{Nu}_{\omega} \propto \mathrm{Ta}^{0.38}$. In contrast, when the pressure forces take over, we have $\mathrm{Nu}_{\omega} \propto \mathrm{Ta}^{0.5}$. Therefore, in the spirit of GL theory of $\mathrm{RB}$ 179, we combine these contributions to set

$$
\mathrm{Nu}_{\omega}=a \mathrm{Ta}^{0.38}+b \mathrm{Ta}^{0.5} \approx c \mathrm{Ta}^{\gamma_{m}}
$$

where $a=\mathrm{Nu}_{v} / \mathrm{Ta}^{0.38}$ and $b=\mathrm{Nu}_{p} / \mathrm{Ta}^{0.5}$ are the prefactors of the separated scalings for $\mathrm{Nu}_{v}$ and $\mathrm{Nu}_{p}$, respectively, which are roughness height dependent, and $\gamma_{m}$ is the effective local exponent predicted by the model. Here for the $h=0.075 d$ case we use the separation shown in Fig. 3.6 (c) at $\mathrm{Ta}=4.6 \times 10^{8}$ to determine $a, b$, and hence the effective exponent $\gamma_{m}$ (other Ta can also be used and the results are similar). It can be seen that the model gives very good agreement with the DNS and EXP values (Fig. 3.6 (d)). Clearly, different number of roughness elements can tune the scaling exponents and optimal transport properties, thus paving the way to control ultimate turbulence. The insight gained from this study provides valuable guidance not only for shear flows but also for thermally driven turbulence with wall roughness in the ultimate regime, which is useful for a wide range of applications in industrial, geophysical, meteorological, and oceanographical flows.

\subsection{Methods}

\subsubsection{Experimental methods}

The experiments were performed in the Twente Turbulent Taylor-Couette facility $\left(\mathrm{T}^{3} \mathrm{C}\right)[3$, consisting of two independently rotating concentric cylinders. The setup 


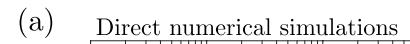

Experiments

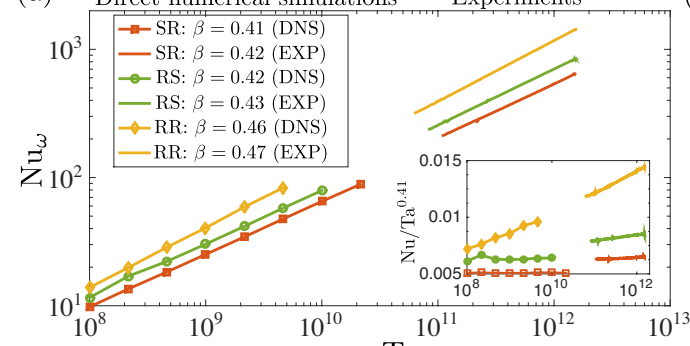

(c)

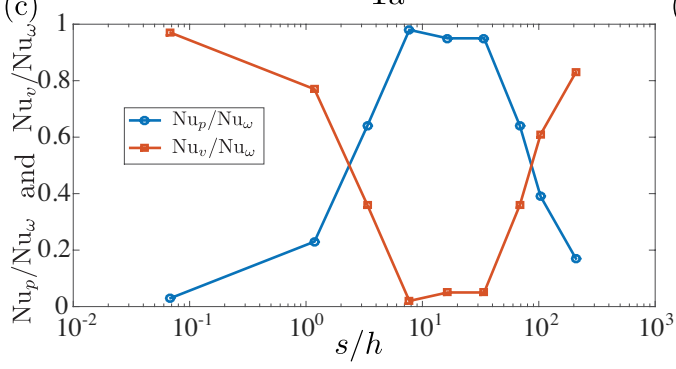

(b)

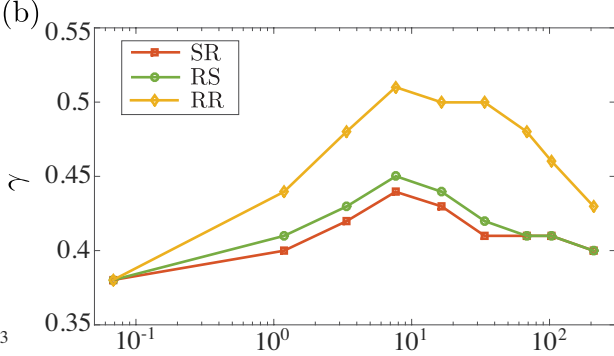

(d)

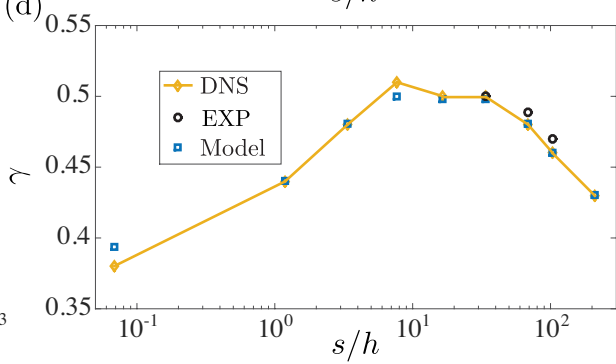

Figure 3.6: Dependence on the roughness density. a, The dimensionless torque as a function of Taylor number Ta: DNS (left part), and experiments (right part) for the case of two ribs with height $h=0.075 d$. For the RR case, the asymptotic ultimate regime is not yet achieved in this situation, in contrast to Fig. 2, when there are six ribs, for which the exponent is 0.5 . b, Effective scaling exponent $\gamma$ for varying spacing distance $s / h$ between the ribs, where $h$ is the height of the roughness. The number of ribs varies from 1 to 196 and correspondingly, the spacing $s / h$ varies from 208.44 to 0.07 at the inner cylinder. To get each $\gamma$, five simulations between $\mathrm{Ta}=10^{8}$ and $\mathrm{Ta}=10^{9}$ were performed. c, Contributions $\mathrm{Nu}_{p}$ (from pressure drag) and $\mathrm{Nu}_{v}$ (from viscous drag) to the global $\mathrm{Nu}_{\omega}$ at $\mathrm{Ta}=4.6 \times 10^{8}$ with varying the spacing $s / h$ between the ribs. The data are collected from DNS. The separation into the two parts is performed at the inner cylinder for the RR case. Clearly, when the pressure forces are dominant, $\gamma$ is closer to $1 / 2$ and when viscous forces are dominant, $\gamma$ is closer to 0.38 (Fig. 3.6 (b)). d, Comparison of the effective scaling exponent $\gamma$ between the DNS results (RR case), EXP results (RR case), and the model results (based on Eq. 3.7) with varying spacing $s / h$ between the ribs. 
has an inner cylinder with a radius of $r_{i}=200 \mathrm{~mm}$ and an outer cylinder with a radius of $r_{o}=279.4 \mathrm{~mm}$, resulting in a radius ratio of $\eta=r_{i} / r_{o}=0.716$ and a gap width of $d=r_{o}-r_{i}=79.4 \mathrm{~mm}$. The gap is filled with water with a temperature of $\mathrm{T} \approx 20^{\circ} \mathrm{C}$. In this chapter, the inner and outer cylinder rotate up to $\omega_{i} /(2 \pi)=7.5 \mathrm{~Hz}$ and $\omega_{o} /(2 \pi)=5 \mathrm{~Hz}$, respectively, resulting in Reynolds numbers up to $\operatorname{Re}_{i}=\omega_{i} r_{i} d / \nu=7.5 \times 10^{5}$ and $\operatorname{Re}_{o}=\omega_{o} r_{o} d / \nu=7 \times 10^{5}$. The cylinders have a height of $L=927 \mathrm{~mm}$, resulting in an aspect ratio of $\Gamma=L /\left(r_{o}-r_{i}\right)=11.7$. The end plates rotate with the outer cylinder. The cylinders were made rough by attaching 2 , 3 , or 6 vertical strips with a square cross-section (four roughness heights: $2 \times 2 \mathrm{~mm}$, i.e. $2.5 \%$ of the gap width, $4 \times 4 \mathrm{~mm}$, i.e. $5 \%$ of the gap width, $6 \times 6 \mathrm{~mm}$, i.e. $7.5 \%$ of the gap width, and $8 \times 8 \mathrm{~mm}$, i.e. $10 \%$ of the gap width) over the entire height on none, both or either one of the cylinders.

The torque is measured with a co-axial torque transducer (Honeywell 2404-5K, maximum capacity of $565 \mathrm{Nm}$ ), located inside the inner cylinder, to avoid measurement errors due to seals and bearing friction, as shown in Supplementary Fig. S1. For the SS case, the inner cylinder consisted of 3 different compartments, in which torque was measured in the middle section to exclude end plate effects. For the rough cases, we measure torque over the entire height of the cylinder.

Planar particle image velocimetry (PIV) measurements were performed in the $\theta-r$ plane at mid-height $(z=L / 2)$. We used a high-resolution sCMOS camera (pco.edge camera with $2560 \mathrm{px} \times 2160 \mathrm{px}$ resolution), which was operated in double frame mode, as depicted in Supplementary Fig. S1. We recorded images through transparent windows in the bottom plate. The flow was illuminated from the side with a pulsed laser (532 nm Quantel Evergreen $145 \mathrm{Nd}$ :YLF). The water was seeded with $20 \mu \mathrm{m}$ fluorescent polymer particles (PMMA-RhB-10 by Dantec). The sheet thickness was approximately $1 \mathrm{~mm}$. The PIV measurements were processed using an iterative multi-pass method with final interrogation windows of 32 pixel $\times 32$ pixel with $50 \%$ overlap and averaged over 500 image pairs per measurement. This results in the averaged azimuthal velocity profile $\left\langle u_{\theta}(r)\right\rangle$.

\subsubsection{Numerical methods}

The motion of the fluid is governed by the incompressible Navier-Stokes equations in the frame co-rotating with the outer cylinder

$$
\begin{aligned}
\frac{\partial \mathbf{u}}{\partial t}+\mathbf{u} \cdot \nabla \mathbf{u} & =-\nabla p+\frac{f(\eta)}{\mathrm{Ta}^{1 / 2}} \nabla^{2} \mathbf{u}-\mathrm{Ro}^{-1} \mathbf{e}_{z} \times \mathbf{u}, \\
\nabla \cdot \mathbf{u} & =0,
\end{aligned}
$$

where $\mathbf{u}$ and $p$ are the fluid velocity and pressure, respectively. $f(\eta)$ is a geometrical factor which has the form

$$
f(\eta)=\frac{(1+\eta)^{3}}{8 \eta^{2}}
$$


Ta is the Taylor number and Ro the Rossby number which characterizes the strength of the driving force. The rotation ratio $a=-\omega_{o} / \omega_{i}$ can alternatively be expressed as Rossby number

$$
\mathrm{Ro}^{-1}=\frac{2 \omega_{o} d}{\left|\omega_{i}-\omega_{o}\right| r_{i}}=-2 \frac{1-\eta}{\eta} \frac{a}{|1+a|} .
$$

The inner cylinder Reynolds number $\operatorname{Re}_{i}=r_{i} \omega_{i} d / \nu$ and outer cylinder Reynolds number $\operatorname{Re}_{o}=r_{o} \omega_{o} d / \nu$ are associated with Ta and Ro through

$$
\operatorname{Re}_{i}=\frac{\mathrm{Ta}^{1 / 2}}{f(\eta)}\left(1+\frac{\eta \mathrm{Ro}^{-1}}{2(1-\eta)}\right)
$$

and

$$
\operatorname{Re}_{o}=\frac{\operatorname{Ro}^{-1} \mathrm{Ta}^{1 / 2}}{2 f(\eta)(1-\eta)} .
$$

The governing equations are solved using an energy conserving second-order finitedifference code [124], in combination with an immersed-boundary method 122,123] to deal with the roughness. To achieve high performance computation, a two-dimensional MPI decomposition technique (MPI-pencil) 121] is adopted. Weak and strong scaling tests show the linear behaviour of the code up to $64 \mathrm{~K}$ cores. The axial direction is periodic and thus the end plate effects [120] are eliminated. The radius ratio is chosen as $\eta=0.716$. The aspect ratio of the computational domain $\Gamma=L / d$, where $L$ is the axial periodicity length, is taken as $\Gamma=2.09$. The ribs are equi-distributed in the azimuthal direction, similarly to the experimental implementation (with one more roughness height at $1.5 \%$ of the gap width). The computation box is tested to be large enough to capture the sign changes of the azimuthal velocity autocorrelation at the mid-gap, as suggested as a criterion for the box size 119. An appropriate number of grid points is chosen to make sure that enough resolution has been employed, e.g. at $\mathrm{Ta}=2.15 \times 10^{9}$ for the $\mathrm{RR}$ case with 6 ribs at roughness height $10 \%$ of the gap width, $3072 \times 1536 \times 1536$ grid points are used.

\subsubsection{Extention of the Grossmann-Lohse theory to the case with wall roughness}

To explain the asymptotic ultimate scaling $1 / 2$ found in this chapter, we first recall the origin of the logarithmic correction. We take the only inner rotation case as an example. We first look at the local dissipation rate in the turbulent boundary layer 39, which can be approximated by

$$
\epsilon_{u}(y)=u_{\tau}^{3} /(\kappa y)
$$

where $u_{\tau}=\sqrt{\tau /\left(2 \rho \pi r^{2} L\right)}$ is the friction velocity, with $\rho$ the fluid density, $\kappa$ the von Kármán constant. The radius $r$ can be either the inner cylinder radius $r_{i}$ or the outer 
one $r_{o}$, and $y$ the distance from the wall. $u_{\tau}$ is connected with the inner cylinder velocity $U=r_{i} \omega_{i}$ through the law of the wall, which is shown to obey

$$
\frac{u_{\tau}}{U}=\frac{\kappa}{\ln \left(B \operatorname{Re}_{i} u_{\tau} / U\right)} .
$$

$\operatorname{Re}_{i}$ is the inner cylinder Reynolds number and which for pure inner cylinder rotation can be related to Ta through the expression $\mathrm{Ta}=\frac{(1+\eta)^{6}}{64 \eta^{4}} \mathrm{Re}_{i}^{2}$, and $B$ is a constant depending on the system geometry. By averaging the local dissipation rate along the radius, we can estimate the mean dissipation rate as

$$
\begin{aligned}
\epsilon_{u, m} & \propto \frac{1}{d / 2} \int_{0}^{d / 2} \epsilon_{u}(y) d y \\
& =\nu^{3} d^{-4} \operatorname{Re}_{i}^{3} \mathcal{L}\left(\operatorname{Re}_{i}\right) \\
& =\nu^{3} d^{-4} \operatorname{Re}_{i}^{3}\left(\frac{u_{\tau}}{U}\right)^{3} \frac{2}{\kappa} \ln \left(\operatorname{Re}_{i} \frac{u_{\tau}}{U} \frac{1}{2}\right)
\end{aligned}
$$

Here we assume that logarithmic boundary layer extends from the wall to the midgap. Usually how far the log-layer extends depends on $\mathrm{Re}_{i}$ and can be a small fraction of the gap width, but still for both TC and pipe flows, taking the half gap width or radius is a reasonable approximation to derive the friction laws. The term $\mathcal{L}\left(\operatorname{Re}_{i}\right)=$ $\left(u_{\tau} / U\right)^{3} \ln \left(\operatorname{Re}_{i} u_{\tau} / U\right)$, depending on $\operatorname{Re}_{i}$, is the logarithmic correction. Using the well known exact relation between $\epsilon_{u, m}$ and $\mathrm{Nu}_{\omega}$, namely

$$
\epsilon_{u, m}=\nu^{3} d^{-4} \mathrm{Ta}\left(\mathrm{Nu}_{\omega}-1\right)\left(\frac{\sqrt{\eta}}{(1+\eta) / 2}\right)^{8}
$$

and with Ta $\propto \operatorname{Re}_{i}^{2}$, one obtains

$$
\frac{\epsilon_{u, m}}{\nu^{3} d^{-4}} \propto \operatorname{Re}_{i}^{3} \mathcal{L}\left(\operatorname{Re}_{i}\right) \text { and } \mathrm{Nu}_{\omega} \propto \mathrm{Ta}^{1 / 2} \mathcal{L}\left(\operatorname{Re}_{i}\right)
$$

with the logarithmic correction $\mathcal{L}\left(\mathrm{Re}_{i}\right)$ for both dissipation rate and torque scalings. It leads to a less steep increase of $\epsilon_{u}$ with increasing $\mathrm{Re}_{i}$ than in the Kolmogorov bulk which scales as $\operatorname{Re}_{i}^{3}$, and hence decreases the torque scaling between $\mathrm{Nu}_{\omega}$ and Ta from the asymptotic ultimate scaling $1 / 2$ to the effective scaling 0.38 , as mentioned before.

With both walls roughened, the log-law in the fully rough regime $\left(u_{\tau} h / \nu>70\right.$; most of the rough cases in this study are in this regime) becomes

$$
\frac{u_{\tau}}{U}=\frac{\kappa}{\ln (B d / h)} \text {. }
$$

The momentum transfer between the wall and the fluid is accomplished by the shear, which in the fully rough regime occurs predominantly by the pressure forces on the side surfaces of the rough elements, rather than by viscous forces. That in the ultimate regime the kinematic viscosity $\nu$ is an irrelevant parameter, is reflected in the velocity 
profile (Eq. (3.19) ) being independent of $\mathrm{Re}_{i}$. Replacing the velocity profile from the smooth one to the rough one in Eqs. 3.14, 3.15, 3.16, remarkably we find that the logarithmic correction term for $\epsilon_{u, m}$ turns into a constant and thus its effect on the scaling exponent vanishes. The mean dissipation rate and torque thus now scale as

$$
\frac{\epsilon_{u, m}}{\nu^{3} d^{-4}} \propto \operatorname{Re}_{i}^{3} \text { and } \mathrm{Nu}_{\omega} \propto \mathrm{Ta}^{1 / 2}
$$

which explains the asymptotic ultimate regime scaling seen in Fig. 2 for the RR case. In the RS or SR case, the boundary layer at the smooth wall depends on $\mathrm{Re}_{i}$ while the boundary layer at the rough wall is independent of it. Therefore, in these cases the logarithmic correction is reduced but not totally canceled. 


\section{Chapter 4}

\section{Rough wall turbulent Taylor-Couette flow: the effect of the rib height ${ }^{1}$}

In this study, we combine experiments and direct numerical simulations to investigate the effects of the height of transverse ribs at the walls on both global and local flow properties in turbulent Taylor-Couette flow. We create rib roughness by attaching up to 6 axial obstacles to the surfaces of the cylinders over an extensive range of rib heights, up to blockages of $25 \%$ of the gap width. In the asymptotic ultimate regime, where the transport is independent of viscosity, we emperically find that the prefactor of the $\mathrm{Nu}_{\omega} \propto \mathrm{Ta}^{1 / 2}$ scaling (corresponding to the drag coefficient $C_{f}(\mathrm{Re})$ being constant) scales with the number of ribs $N_{r}$ and by the rib height $h^{1.71}$. The physical mechanism behind this is that the dominant contribution to the torque originates from the pressure forces acting on the rib which scale with rib height. The measured scaling relation of $N_{r} h^{1.71}$ is slightly smaller than the expected $N_{r} h^{2}$ scaling, presumably because the ribs cannot be regarded as completely isolated but interact. In the counter-rotating regime with smooth walls, the momentum transport is increased by turbulent Taylor vortices. We find that also in the presence of transverse ribs these vortices persist. In the counter-rotating regime, even for large roughness heights, the momentum transport is enhanced by these vortices.

\footnotetext{
${ }^{1}$ Ruben A. Verschoof, Xiaojue Zhu, Dennis Bakhuis, Sander G. Huisman, Roberto Verzicco, Chao Sun, an Detlef Lohse, Rough wall turbulent Taylor-Couette flow: the effect of the rib height, under review.

Experiments by Verschoof and Bakhuis. Simulations by Zhu. Data analysis by Verschoof and Zhu. Verschoof wrote the paper. Verzicco, Sun and Lohse supervised the project. All authors discussed the results and proofread the paper.
} 


\subsection{Introduction}

Turbulent flows with rough walls are omnipresent in nature and industry. In fact, for increasing Reynolds numbers, the viscous length-scales in the flow decrease, and eventually every surface appears to be rough even when the roughness is small in absolute scale. Roughness in turbulent flows is relevant in many fields, one can think of e.g. biofouling in marine vessels [85], atmospheric boundary layers [90, and the accelerated transition to turbulence, see e.g. refs. 45, 151] for recent reviews. The study of roughness in turbulent flows has received tremendous attention, especially the field of rough pipe flow studies has a long history. The most seminal work to date remains the well-known pipe flow experiments by Nikuradse 145 . He expressed the friction as a dimensionless friction factor $C_{f}$, and found that $C_{f}$ decreases for increasing Reynolds number Re, and eventually becomes constant in the presence of roughness. The absolute value of $C_{f}$ then depends on the characteristic height of the roughness. Using that work and successive work of Colebrook 83] and Moody 84], engineers were enabled to estimate the pressure drop in pipes. However, the influence of roughness on turbulent flows remains far from being understood. Many experimental studies focussed on industrial applicability, and have not emphasized the physical understanding of the flow dynamics, as was pointed out by ref. [150]. Furthermore, roughness remains hard to quantify given the huge variety of roughness types. Although significant progress has been made in recent years, the study of roughness in highly turbulent flows remains a topic of great interest to both physicists and engineers $86,89,91,92,147$.

In this study, building further upon our recent work 220], we use a Taylor-Couette system, i.e. the fluid flow between two concentric, independently rotating cylinders, to study the effects of transverse ribs in highly turbulent flow. Taylor-Couette (TC) flow has the advantages of (i) being a closed flow with an exact balance between energy input and dissipation, (ii) being accesible to study both numerically and experimentally due to its simple geometry and high symmetries, (iii) having no streamwise spatial transients and (iv) being mathematically well-defined based on the Navier-Stokes equations, the continuity equation and the known boundary conditions.

Indeed, Taylor-Couette flow, together with pipe flow and Rayleigh-Bénard (RB) convection, is one of the canonical systems in which the physics of fluids is studied $50,51,94$. When the correct dimensionless parameters are used, the scaling relations between driving and response are the same for $\mathrm{RB}$ convection and $\mathrm{TC}$ flow, namely 186

$$
\mathrm{Nu}_{\omega} \propto \mathrm{Ta}^{1 / 2} \mathcal{L}(\mathrm{Re}), \quad \text { and } \quad \mathrm{Nu} \propto \mathrm{Ra}^{1 / 2} \mathcal{L}(\mathrm{Re}),
$$

for fixed (geometric) Prandtl number. The terms $\mathcal{L}(R e)$ are logarithmic corrections, which are related to a viscosity-dependence in the turbulent boundary layers 185 . 186 . For smooth walls, effective power laws of $\mathrm{Nu}_{\omega} \propto T a^{0.38}$ and $\mathrm{Nu} \propto \mathrm{Ra}^{0.38}$ are found both numerically and experimentally in TC flow and RB convection in hitherto studied parameter regions $\left(10^{8} \leq \mathrm{Ta} \leq 10^{13}\right.$ and $\left.5 \times 10^{14} \leq \mathrm{Ra} \leq 10^{15}\right)$, $4,5,153,154$.

Recently, building on prior work 96, 125] we showed that attaching ribs on both 
cylinders in a TC setup is an effective way to attain a $\mathrm{Nu}_{\omega} \propto \mathrm{Ta}^{1 / 2}$ scaling without log-corrections 220]. Thanks to the ribs, the viscosity-dependence in the boundary layers is eliminated as the dissipative process becomes fully pressure-dominated. This scaling, which we called "asymptotic ultimate turbulence scaling" has the same scaling as the mathematical upper bound of momentum transport [156,157, namely $\mathrm{Nu}_{\omega} \propto$ $\mathrm{Ta}^{1 / 2}$, where the prefactor of the $\mathrm{Nu}_{\omega} \propto \mathrm{Ta}^{\gamma}$ scaling still depends on the roughness characteristics, i.e. the height and number of ribs. Similar observations were made in pipe flow 100, as $\mathrm{Nu}_{\omega} \propto \mathrm{Ta}^{1 / 2}$ is mathematically equivalent to having a Reynolds number independent drag coefficient $C_{f}$. In fact, what we here refer to as "asymptotic ultimate turbulence" is identical to the so-called "fully rough" regime in pipe flow.

The geometry of a Taylor-Couette setup is characterized by two geometric parameters, which are the radius ratio $\eta=r_{i} / r_{o}$ and the aspect ratio $\Gamma=L /\left(r_{o}-r_{i}\right)$, in which $r_{i}$ and $r_{o}$ are the radii of the inner and outer cylinder, respectively, and $L$ is the height of the setup. Taylor-Couette flow is driven by the rotation of one or both cylinders. Their driving can be expressed as two different Reynolds numbers, i.e.

$$
\operatorname{Re}_{i}=\frac{\omega_{i} r_{i} d}{\nu}, \quad \text { and } \quad \operatorname{Re}_{o}=\frac{\omega_{o} r_{o} d}{\nu},
$$

in which $\nu$ is the kinematic viscosity, $d=r_{o}-r_{i}$ is the gap width and $\omega_{i}$ and $\omega_{o}$ are the angular velocities of the inner and outer cylinders, respectively. Alternatively, we can express the driving using the Taylor number Ta and a rotation ratio. The Taylor number, being equivalent to the Rayleigh number in RB convection, is given as

$$
\mathrm{Ta}=\frac{\sigma}{4} \frac{d^{2}\left(r_{i}^{2}+r_{o}^{2}\right)\left(\omega_{i}-\omega_{o}\right)^{2}}{\nu^{2}} \propto\left(\operatorname{Re}_{i}-\eta \operatorname{Re}_{o}\right)^{2} .
$$

Here $\sigma=\left(\frac{1+\eta}{2 \sqrt{\eta}}\right)^{4}$, which is a fixed geometric parameter, is referred to as a 'geometric Prandtl number' 189]. The rotation ratio between both cylinders is given as $a=$ $-\omega_{o} / \omega_{i}$, and can also be expressed in terms of an inverse Rossby number, which directly enters the equations of motion as the Coriolis force, as will be discussed in section 4.2 .2 The rotation ratio $a$ and the inverse Rossby number are related by

$$
\mathrm{Ro}^{-1}=\frac{2 \omega_{o} d}{\left|\omega_{i}-\omega_{o}\right| r_{i}}=-2 \frac{a}{|1+a|} \frac{1-\eta}{\eta} .
$$

The primary response parameter is the torque $\tau$ necessary to rotate the cylinders at a given driving $R e_{i}$ and $R e_{o}$, or, equivalently, $T a$ and $a$. To underline the analogy with RB convection, the torque is expressed as the Nusselt number, which is the ratio between the angular velocity flux $J^{\omega}$ and its laminar value $J_{\text {lam }}^{\omega}=2 \nu r_{i}^{2} r_{o}^{2}\left(\omega_{i}-\right.$ $\left.\omega_{o}\right) /\left(r_{o}^{2}-r_{i}^{2}\right)$. The Nusselt number is directly related to the torque by

$$
\mathrm{Nu}_{\omega}=J^{\omega} / J_{\text {lam }}^{\omega}=\frac{\tau}{2 \pi L \rho J_{\text {lam }}^{\omega}},
$$

in which $\rho$ is the density of the fluid. These equations hold for all cases, including co- and counter-rotation, and are also valid when ribs are added to the cylinders. 
A different nondimensional representation of the torque is as friction coefficient $C_{f}$, which is traditionally used in the wall-bounded turbulence community

$$
C_{f}=\frac{\tau}{L \rho \nu^{2}\left(\operatorname{Re}_{i}-\eta \operatorname{Re}_{o}\right)^{2}}
$$

Lastly, the torque can be related to the friction velocity at either the inner or the outer cylinder $u_{\tau}=\sqrt{\tau / 2 \pi \rho r_{i, o}^{2} L}$, which can be used, along with the viscous lengthscale $\delta_{\nu}=\nu / u_{\tau}$, to express the velocity and wall-normal distance in wall units.

In this work, we build further on our prior work [220], by combining detailed experiments and direct numerical simulations, to quantify the influence of the rib height on the prefactors of the scaling relations. Furthermore, we study the role of the pressure acting on the ribs, as well as the local flow response.

The outline of this chapter is as follows; We first discuss the experimental and numerical methods, as well as the explored parameter space in section 4.2. We continue with presenting global flow results in section 4.3 , which is followed by local flow results in section 4.4. In section 4.5, we explore the regime of counter-rotating cylinders. We conclude this chapter in section 4.6 .

\subsection{Methods}

\subsubsection{Experimental methods}

The experiments were performed in the Twente Turbulent Taylor-Couette $\left(\mathrm{T}^{3} \mathrm{C}\right)$ facility [3] The setup has an inner cylinder with a radius of $r_{i}=200.0 \mathrm{~mm}$ and an outer cylinder with a radius of $r_{o}=279.4 \mathrm{~mm}$, resulting in a radius ratio of $\eta=r_{i} / r_{o}=0.716$ and a gap width of $d=r_{o}-r_{i}=79.4 \mathrm{~mm}$. The gap is filled with water at a temperature of $\mathrm{T}=20 \pm 0.5^{\circ} \mathrm{C}$, which is kept constant by active cooling through the top and bottom plates. Nonetheless, the temperature is monitored continuously, such that the viscosity is calculated using the instantaneous temperature. In this work, the inner and outer cylinder rotate up to $\omega_{i} /(2 \pi)=10 \mathrm{~Hz}$ and up to $\omega_{o} /(2 \pi)= \pm 5 \mathrm{~Hz}$, respectively, resulting in Reynolds numbers up to $\operatorname{Re}_{i}=\omega_{i} r_{i} d / \nu=1 \times 10^{6}$ and $\operatorname{Re}_{o}=\omega_{o} r_{o} d / \nu=7 \times 10^{5}$. The cylinders have a height of $L=927 \mathrm{~mm}$, resulting in an aspect ratio of $\Gamma=L /\left(r_{o}-r_{i}\right)=11.7$. The end plates rotate with the outer cylinder.

The torque is measured with a co-axial torque transducer (Honeywell 2404-1K, maximum capacity of $115 \mathrm{Nm}$ ), located inside the inner cylinder to avoid measurement errors due to friction of seals and bearings, as shown in figure 4.1. In previous studies using this setup, the inner cylinder consisted of 3 different sections, and the torque was measured only in the middle section to reduce end plate effects 4, 176]. Here, we measure over the entire height of the cylinder, which accounts for the slightly different results for the smooth-wall case as compared to those studies. 

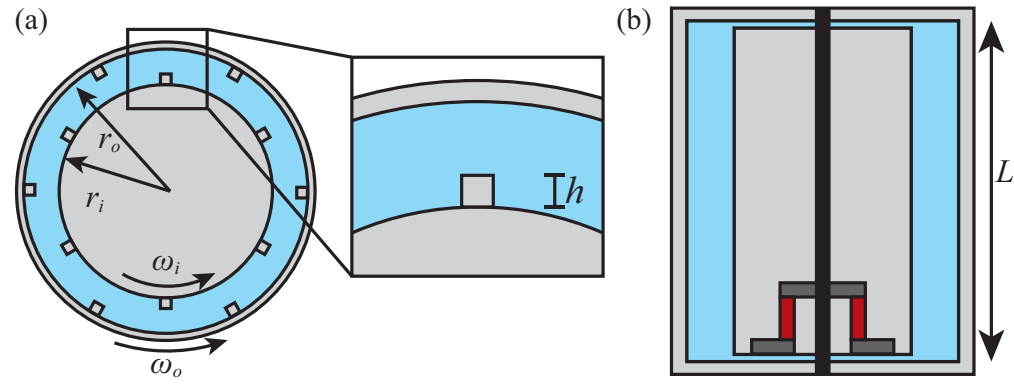

Figure 4.1: Schematic of the experimental setup (a) Top view of the experimental setup, in which ribs (not to scale) are placed on both the inner and outer cylinder. The ribs extend over the entire height of the cylinders. The zoom shows how the rib height $h$ is defined. (b) Cross-section of the TC setup. The torque sensor is located in the inner cylinder.

\subsubsection{Numerical methods}

We numerically solve the incompressible Navier-Stokes equations in the frame of reference which co-rotates with the outer cylinder

$$
\begin{aligned}
\frac{\partial \mathbf{u}}{\partial t}+\mathbf{u} \cdot \nabla \mathbf{u} & =-\nabla p+\frac{f(\eta)}{\mathrm{Ta}^{1 / 2}} \nabla^{2} \mathbf{u}-\mathrm{Ro}^{-1} \mathbf{e}_{z} \times \mathbf{u}, \\
\nabla \cdot \mathbf{u} & =0
\end{aligned}
$$

where $\mathbf{u}$ is the fluid velocity, $p$ the pressure, and $\mathbf{e}_{z}$ the unit vector in the axial direction. $f(\eta)$ is a geometrical factor which has the form

$$
f(\eta)=\frac{(1+\eta)^{3}}{8 \eta^{2}}
$$

The direct numerical simulations were carried out by solving the above governing equations, using a second order finite difference code AFiD [121, 124], in combination with an immersed-boundary method 122,123 for the rotating roughness elements. A two-dimensional MPI decomposition technique (MPI-pencil) 121] was implemented to achieve highly parallelized computation. In recent years, we have tested the code extensively for TC flow with smooth [5, 55, 119 and rough 173,220 walls. The boundary condition in the axial direction is periodic and thus we do not have end plate effects, which, as ref. [120] showed, are small in the turbulent regime. The radius ratio is chosen as $\eta=0.716$, the same as in experiments. The aspect ratio of the computational domain $\Gamma=L / d$, where $L$ is the axial periodicity length, is taken as $\Gamma=2.09$. The azimuthal extent of the domain is $\pi / 3$, to reduce computation costs without affecting the results [171]. The computation box is tested to be large enough to capture the sign changes of the azimuthal velocity autocorrelation at the mid-gap, which was suggested as a criterion for the box size [119]. For more information on the numerical details, we refer to ref. 220. 

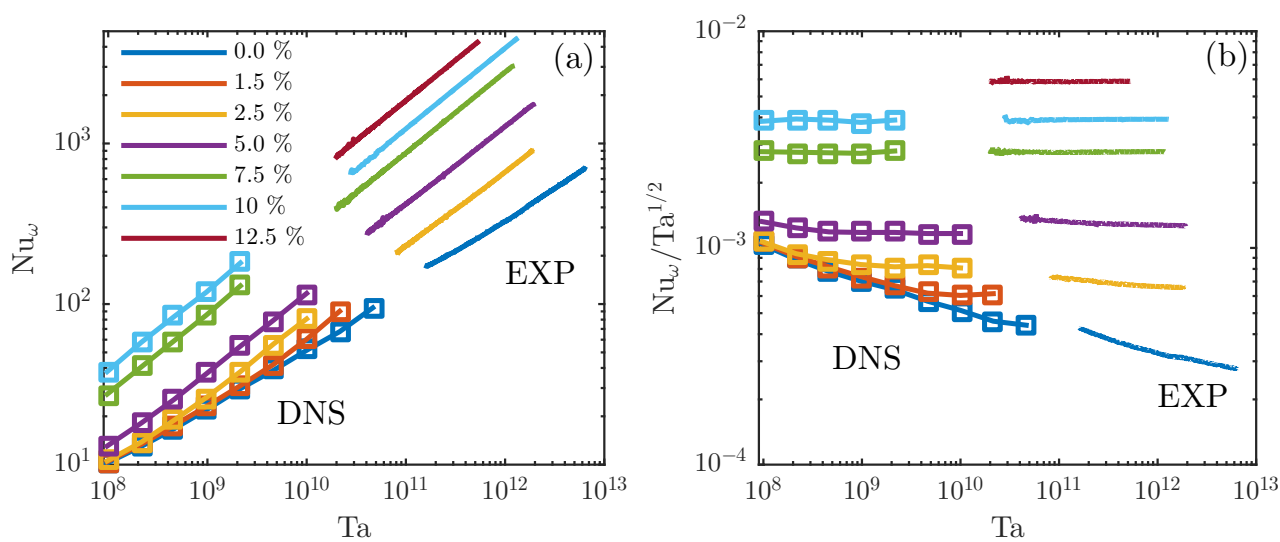

Figure 4.2: Torque scaling as a function of driving for $a=0$, i.e. pure inner cylinder rotation. 6 ribs are attached to each cylinders. As indicated in the figure, the lower Taylor number data points are DNSs, the higher Taylor number data are experimental results. (a) The Nusselt number $\mathrm{Nu}_{\omega}$ as a function of Taylor number Ta. (b) Here we compensate $\mathrm{Nu}_{\omega}$ with $\mathrm{Ta}^{1 / 2}$ to reveal whether the asymptotic ultimate regime is reached. The local scaling depends on both the Taylor number and the roughness height.

\subsubsection{Explored parameter space}

Experimentally, we explored Taylor numbers of $O\left(10^{10}\right)$ to $O\left(10^{13}\right)$. Numerically, all Taylor numbers below $O\left(10^{10}\right)$ are accessible. The exact values depend on the roughness size. In that sense, the simulations and experiments are completely complementary, and we explore a parameter space in which Ta extends over 5 orders of magnitude. We restrict ourselves to, (i) equidistant ribs, and (ii) the same number of ribs on both the inner and outer cylinder. Numerically, we attach 6 ribs to both cylinders. The used rib heights are $1.5 \%, 2.5 \%, 5 \%, 7.5 \%$ and $10 \%$ of the gap width. The maximum blockage obviously is twice as large: e.g. with when 2 ribs with a $10 \%$ rib height pass, the local blockage is $20 \%$ of the gap width.

Experimentally, we attach 2,3 , or 6 vertical ribs to both cylinders, as shown in figure 4.1 . The used roughness heights are $2 \mathrm{~mm}, 4 \mathrm{~mm}, 6 \mathrm{~mm}, 8 \mathrm{~mm}$, and $10 \mathrm{~mm}$, corresponding to $2.5 \%, 5 \%, 7.5 \%, 10 \%$, and $12.5 \%$ of the gap width. For both the simulations and experiments, we also measure without ribs as reference smooth-wall case. Numerically, we restricted ourselves to inner cylinder rotation only, whereas experimentally we also explore the counter-rotating regime.

\subsection{Global response: torque and its scaling}

The global response of momentum transport in TC flow can be expressed as the torque which is necessary to keep the cylinders rotating at fixed angular velocities. Here we show the dimensionless torque $\mathrm{Nu}_{\omega}$ as a function of driving, expressed here 

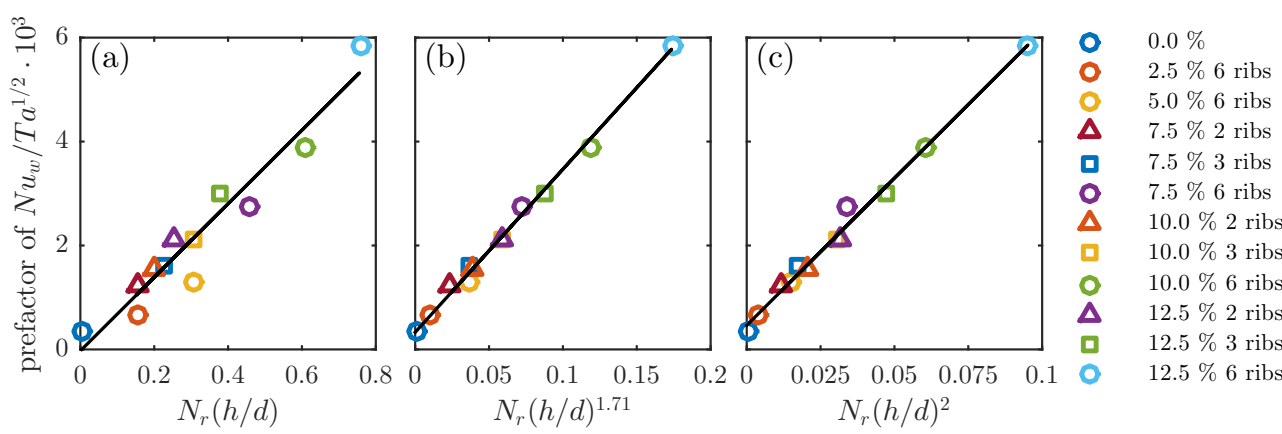

Figure 4.3: Prefactor of the $\mathrm{Nu}_{\omega} \propto \mathrm{Ta}^{1 / 2}$ scaling as a function of rib number $N_{r}$ and normalized rib height $h / d$, obtained from experiments. (a) Results as a function of rib frontal area, which equals the $N_{r}(h / d)$. In (b), we show the best fit, showing that the prefactor scales with $N_{r}(h / d)^{1.71}$. In (c), we show the prefactor as a function of $N_{r}(h / d)^{2}$. The goodness of the fits is calculated here with the $R^{2}$ value. For figures (a), (b), and (c), they are $R_{b=1}^{2}=0.9317, R_{b=1.71}^{2}=0.9953$, and $R_{b=2}^{2}=0.9901$, respectively.

as the Taylor number Ta. In figure 4.2 we show results for 6 ribs on both cylinders for the case with a stationary outer cylinder. This figure clearly shows that $\mathrm{Nu}_{\omega}$ is increased tremendously as the roughness height increases. To highlight the local scaling, we compensate $\mathrm{Nu}_{\omega}$ with $\mathrm{Ta}^{1 / 2}$ in figure $4.2 \mathrm{p}$. This figure is very similar to the well-known Moody diagram for pipe flow, in which the friction coefficient $C_{f}$ is expressed as a function of the Reynolds number 84,220 . In fact, using the definitions given above, and apart from a prefactor, $\mathrm{Nu}_{\omega} / \mathrm{Ta}^{1 / 2}$ and $C_{f}$ are identical, as they are related as

$$
\frac{\mathrm{Nu}_{\omega}}{\mathrm{Ta}^{1 / 2}}=\frac{(1-\eta)\left(1-\eta^{2}\right)}{2 \pi \sqrt{\sigma} \sqrt{\eta^{2}+1}} C_{f}=\frac{2(\eta-1)^{2} \eta}{\pi(1+\eta) \sqrt{1+\eta^{2}}} C_{f}
$$

For the currently used radius ratio of $\eta=0.716$, this results in $\mathrm{Nu}_{\omega} / \mathrm{Ta}^{1 / 2}=$ $0.0174 C_{f}$. Here, we see that for the currently studied Taylor number regime, all cases with ribs larger than $7.5 \%$ of the gap width result in reaching the asymptotic ultimate regime 220], i.e. the $\mathrm{Nu}_{\omega} \propto \mathrm{Ta}^{1 / 2}$ scaling is attained.

To understand how the torque scales with rib height, we extract the mean prefactor of the experimental results shown in figure 4.2 over the measured Taylor number range, as shown in figure 4.3. We here plot experimental results for various roughness heights $h$ and rib number $N_{r}$. Intuitively, one could think that the prefactor scales with the total frontal area of the ribs, i.e. with $S=N_{r} h L$. When considering e.g. the drag equation $F_{D}=\frac{1}{2} \rho u_{\infty}^{2} C_{D} S$, this argument indeed could be correct, as long as $C_{D}$ remains constant. Although figure 4.3 a indeed shows some correlation, the quality of the fit, which is shown as solid black line, can be improved. The fit quality can be increased by assuming a scaling of type $N_{r}(h / d)^{b}$, in which $b$ is a fitting parameter. It is found that $b=1.71$ collapses our data in the best way. The $R^{2}$ values are given 


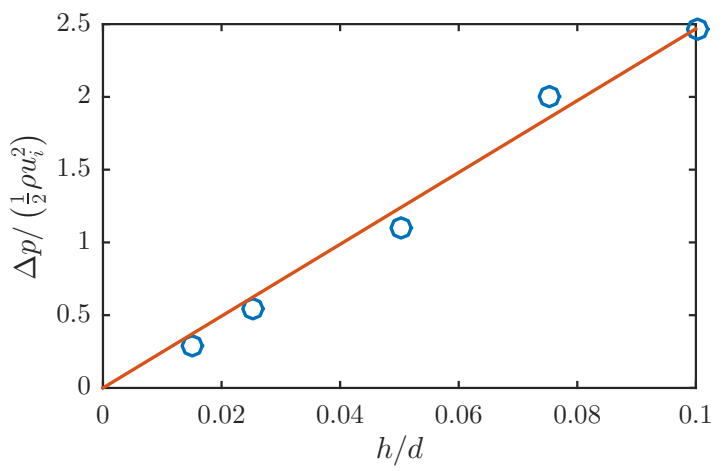

Figure 4.4: Dimensionless pressure difference $\Delta p / \frac{1}{2} \rho u_{i}^{2}$ between upstream and downstream sides of the ribs as a function of rib height $h$ for $\mathrm{Ta}=1 \times 10^{9}$ and $a=0$, obtained from DNS. We observe a linear dependence between pressure difference and rib height.

in de caption of figure 4.3 .

As shown in ref. [220], in the asymptotic ultimate regime, the pressure force results in the dominant injection of momentum, rather than the skin friction. The torque $\tau_{p}$ exerted by the fluid on the inner cylinder through pressure forces on the ribs is given as:

$$
\tau_{p}=r_{i} S \Delta p
$$

in which $S=h N_{r} L$ is the total frontal area of all the ribs, and $\Delta p$ is a mean pressure difference between the upstream and downstream side of the rib. An instantaneous pressure field is shown in figure $4.5 \mathrm{a}$. Obviously, a local region of high pressure is found at the upstream side of the rib, and a local region of low pressure is present at the downstream side. In figure 4.4, we show the pressure difference $\Delta p$ as a function of rib height $h$. Indeed, the pressure difference is related to the rib height, and, is surprisingly well represented by the linear relation $\Delta p \propto h$. With this knowledge we can now better understand the height dependence of the prefactor of the $\mathrm{Nu}_{\omega} / \mathrm{Ta}^{1 / 2}$ relation: the pressure forces scale with the product frontal area $N_{r} h L$, and pressure difference $\Delta p$, which is also proportional to the rib height $h$, leading to the prefactor to scale with $N_{r}(h / d)^{2}$, as long as the skin friction is negligible compared to the pressure forces, and in the case that ribs are unaffected by the neighbouring ribs. This result is close to what we found in our experiments, where we observe a slightly smaller scaling, namely the aforementioned $N_{r}(h / d)^{1.71}$ scaling, presumably because the ribs cannot be regarded as isolated. In addition, the analysis presented here is obviously limited to cases in which the pressure drag is dominant. Therefore, it does not fully cover cases with too sparse or dense rib densities, as well as too small rib heights 220 . 

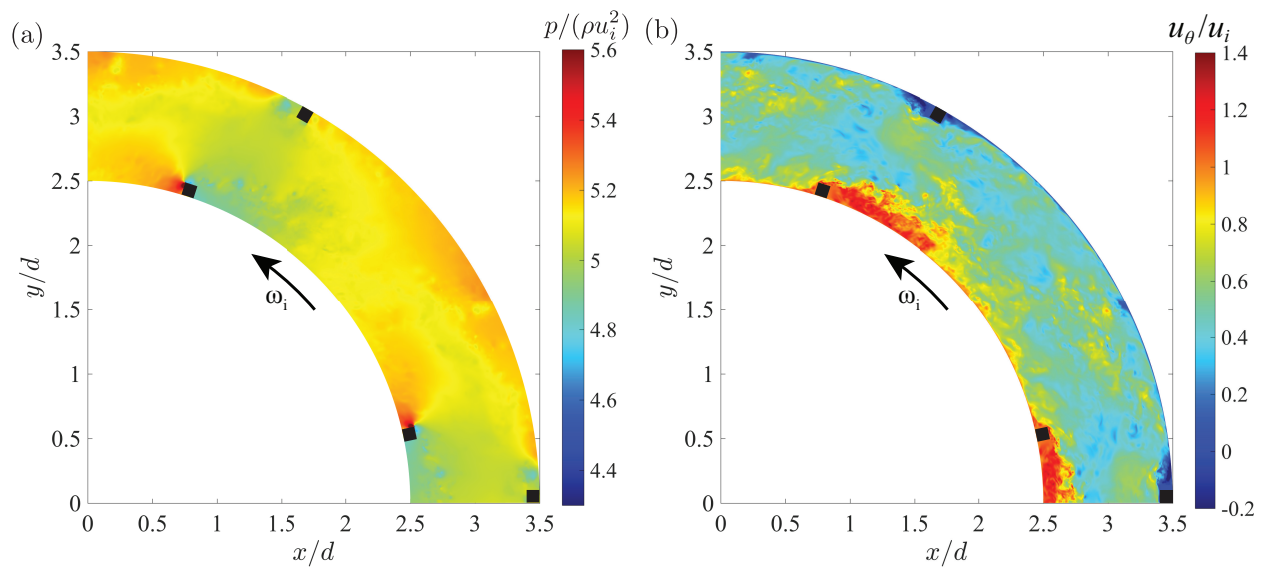

Figure 4.5: (a) Dimensionless pressure field, and (b) dimensionless azimuthal velocity field obtained with DNS. The rib height is $0.1 d$, and the Taylor number is $1 \times 10^{9}$ and $a=0$. We here show one instantaneous field, taken at mid-height of the numerical domain. The inner cylinder rotates counter-clockwise as indicated, the outer cylinder is stationary. The positions of the ribs are indicated by the black squares.

\subsection{Local results}

We now show the azimuthal velocity profiles in figure 4.6. extracted from the DNS simulations which were shown in figure 4.2. Although the mean azimuthal velocity in the bulk remains largely unaffected by the roughness, that is not the case in the boundary layers (BLs), as one could expect for rough walls. Here we see that the BLs become thicker, and consequently that the wall-normal velocity gradient is less steep for all roughness cases. The difference in $u_{\theta}$ in the BLs for all roughness heights is difficult to observe in figure 4.6, and becomes clearer in figure 4.7. In this figure, we show the azimuthal velocity profiles in the form $u^{+}=\left(u_{i}-u_{\theta}\right) / u_{\tau}$ as function of the wall distance $y^{+}=y / \delta_{\nu}$ from the inner cylinder, in which the viscous lengthscale is calculated as $\delta_{\nu}=\nu / u_{\tau}$. We see, as is known for roughness, that $u^{+}$decreases with roughness 151]. One could think that rib roughness has a significantly different influence on the flow than e.g. sand grain roughness, as ribs act in a more local and isolated way than the uniform sand grain roughness. This is however not reflected in the data, neither in the global torque results, nor by these local velocity results, as the results shown here are similar to a wide range of other types of roughness in other flow setups, see e.g. refs. $86,87,147,150]$. Therefore our data suggests that the overall trends are independent to the type of roughness applied.

We clearly see in both figures 4.6 and 4.7 that the shear at the cylinders becomes increasingly smaller with increasing roughness. As the skin friction is directly related to the shear at the wall, we see that this contribution to the torque gets smaller, wheareas the total torque is increased tremendously. This finding again confirms that skin friction is not the dominant way of injecting energy to the system, and again 


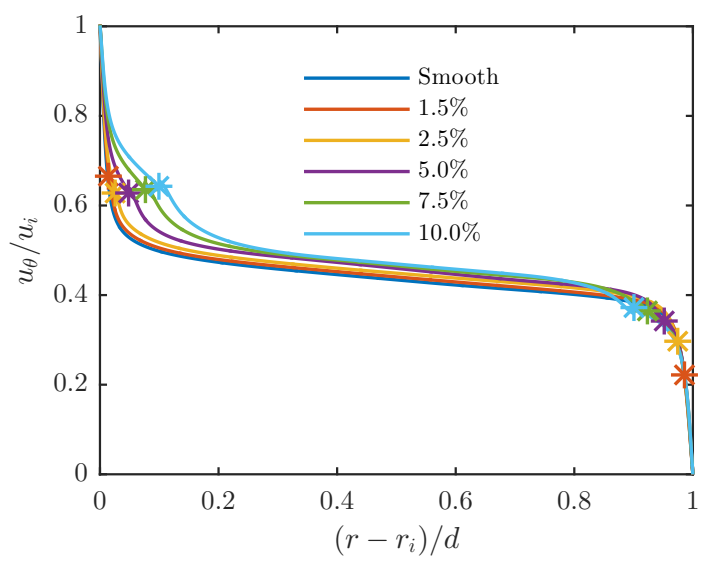

Figure 4.6: Azimuthal velocity profiles for various roughness heights, nondimensionalized by the inner cylinder velocity and the gap width at Ta $=1 \times 10^{9}$, obtained from DNS. Here, six ribs are attached to both cylinders. The outer cylinder is kept stationary. The stars indicate the extent of the ribs.
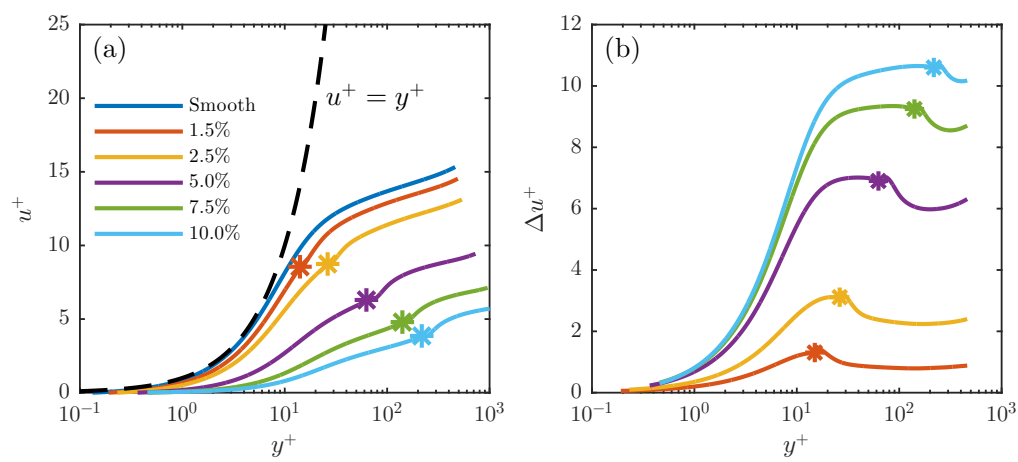

Figure 4.7: (a) Velocity profiles for various roughness heights non-dimensionalized by the friction velocity $u_{\tau}$ and wall distance to the inner cylinder $\delta_{\nu}$ at Ta $=1 \times 10^{9}$, obtained from DNS. Here, six ribs are attached to both cylinders. The outer cylinder is kept stationary. The stars indicate the extent of the ribs. In (b), we show the difference from the smooth case, $\Delta u^{+}=u_{\text {smooth }}^{+}-u^{+}$. 

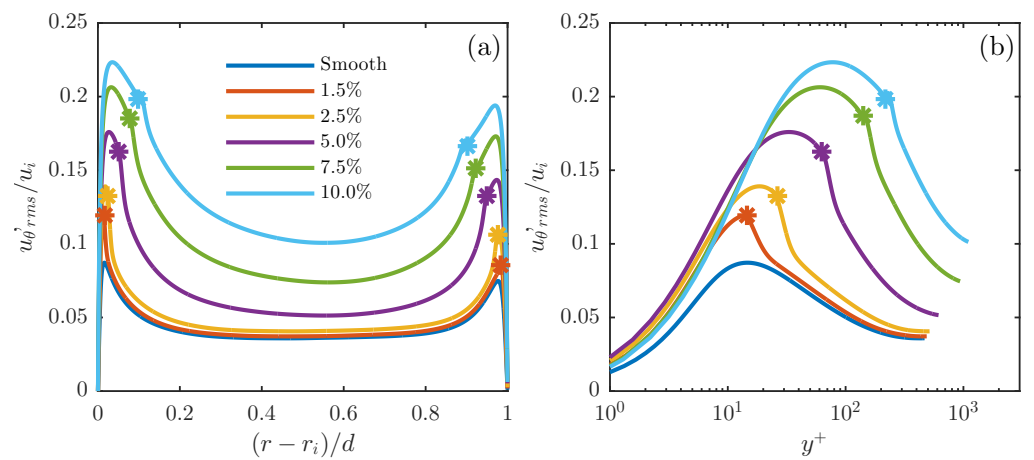

Figure 4.8: RMS of the velocity fluctuations of the azimuthal velocity at Ta $=1 \times 10^{9}$, obtained from DNS. Here, six ribs are attached to both cylinders. The outer cylinder is kept stationary. The stars indicate the extent of the ribs. In (a), the full fluctuation profiles are given, whereas in (b) the fluctuations are shown on a semi-log scale as function of the wall distance to the inner cylinder.

highlights the role of the pressure drag. Velocity fluctuations (fig. 4.8) show a clear and large peak close to both cylinders, with velocities in the bulk being of around $5 \%$ of the mean azimuthal velocity. As the rib size increases, the position of the aforementioned peaks is further away from the cylinder wall, the peak value however being at smaller $y^{+}$values than the roughness height $h^{+}$, indicated in the figure by the asterisk symbols. This indicates that the equivalent sand roughness height $k_{s}$ of the roughness heights is significantly smaller than the rib height itself, as $k_{s}$ is expected to be located closer to the wall than the peak of the fluctuations.

\subsection{Optimal transport}

So far we focussed on cases with stationary outer cylinder. In this section, we explore the behaviour of wall roughness in the counter-rotating regime. For smooth walls, it is known that outer cylinder rotation has a significant influence on the momentum transport between both cylinders [4,5,171]. Counter-rotating cylinders stimulate the existence of so-called "turbulent Taylor vortices", which enhance the momentum transport 5 99. At a rotation ratio of $a_{\text {opt }}=0.36$, the momentum transport reaches a maximum for the currently used radius ratio for the smooth wall case. In both extrema of $a= \pm \infty$, the flow (in the absence of end plates) is laminar, and thus $\mathrm{Nu}_{\omega}=1$ 98, 176. Here we investigate what the influence of ribs is on the behaviour in the counter-rotating regime. To study this, we fixed the Taylor number to $T a=3.8 \times 10^{11}$, and increased the rotation ratio $a$ from $a=0$ to $a=1$ in a quasi-stationary way. As seen in figure 4.9 , there is a momentum transport enhancement in the counter-rotating regime for all cases. When normalizing all curves by their $\mathrm{Nu}_{\omega}(a=0)$ value, their shapes become very similar, i.e. apart from a prefactor, the behaviour is comparable. As in the smooth-wall case this 'optimal transport peak' is related to the Taylor rolls, 

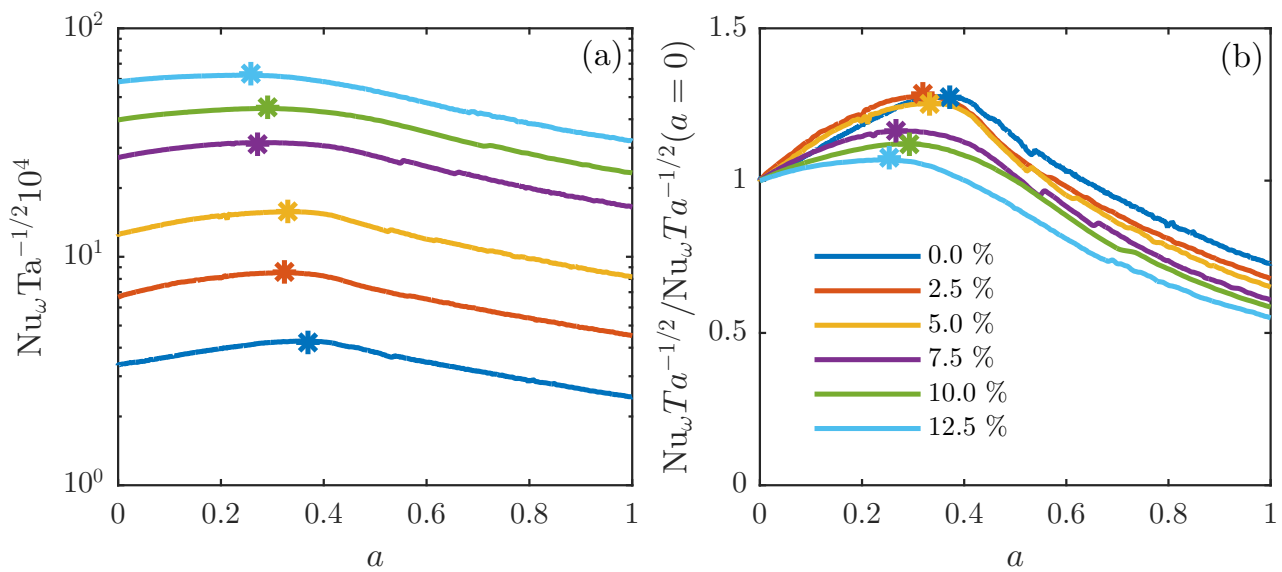

Figure 4.9: (a) The angular momentum transport $\mathrm{Nu}_{\omega}$ as a function of rotation ratio $a$ and various rib heights (see legend) from the experiments. We fixed the number of ribs to 6 . We multiply $\mathrm{Nu}_{\omega}$ with $\mathrm{Ta}^{-0.5}$ to minimize temperature fluctuations in the experiments, which are reflected in the viscosity dependence of the Taylor number. (b) $\mathrm{Nu}_{\omega}(a)$ normalized with its value for $a=0$, to highlight the differences between the shape of the curves. We indicated the maxima of each curve using an asterisk symbol.

these results suggest that also in the presence of roughness Taylor rolls exist. As was shown in ref. 173], ribs effectively shed off turbulent plumes, which feed and drive the Taylor rolls. That even for extremely large roughness heights, here up to $12.5 \%$ of the gap width, these rolls exist is surprising. The peak value of $\mathrm{Nu}_{\omega}(a) / \mathrm{Nu}_{\omega}(a=0)$ however does decrease for increasing rib height, indicating that the relative strength of the Taylor vortices decreases.

\subsection{Conclusions and Outlook}

To conclude, building further upon our recently published work 220, by providing further flow details on the local flow organization, the dependence on rib height and the behaviour in the regime of counter-rotating cylinders is illuminated. We found that the momentum transport is largely enhanced with increasing rib height, caused by increasing pressure forces acting on the ribs. A scaling argument is found which predicts a scaling linear with rib number $N_{r}$ and squared with rib height $h$, i.e. $N_{r}(/ d) h^{2}$. Experimentally, we find that to collapse the data the second scaling exponent must be slightly smaller for the investigated $N_{r}$ and $h$, i.e. $N_{r}(h / d)^{1.71}$, presumably because the ribs cannot be regarded as isolated. Velocity profiles and the near-wall velocities in wall units show that the velocity gradient close to the wall decreases with increasing roughness, similarly to what has been observed in other flow systems using other roughness types. 
In the counter-rotating regime, the momentum transport depends on the rotation ratio similarly as in the smooth-wall case. Therefore, we hypothesize that in spite of the roughness, Taylor rolls still exist, and that their momentum-transporting role remains unaffected by it. However, the ribs might decrease the effective radius, thus increasing the apparent aspect ratio and might thus allow for a larger number of rolls. Furthermore, the characteristics of the rolls can be affected by the significantly increased mixing. In addition, efforts are currently ongoing towards more realistic types of roughness, i.e. 'sand-grain roughness', which are often encountered in turbulent flows encountered in engineering applications and in nature. 
62 CHAPTER 4. ROUGH WALL TURBULENT TAYLOR-COUETTE FLOW 


\section{Part III}

\section{Air lubrication in turbulent flows}




\section{Chapter 5}

\section{Bubble drag reduction requires large bubbles ${ }^{T}$}

In the maritime industry, the injection of air bubbles into the turbulent boundary layer under the ship hull is seen as one of the most promising techniques to reduce the overall fuel consumption. However, the exact mechanism behind bubble drag reduction is unknown. Here we show that bubble drag reduction in turbulent flow dramatically depends on the bubble size. By adding minute concentrations (6 ppm) of the surfactant Triton X-100 into otherwise completely unchanged strongly turbulent Taylor-Couette flow containing bubbles, we dramatically reduce the drag reduction from more than $40 \%$ to about $4 \%$, corresponding to the trivial effect of the bubbles on the density and viscosity of the liquid. The reason for this striking behavior is that the addition of surfactants prevents bubble coalescence, leading to much smaller bubbles. Our result demonstrates that bubble deformability is crucial for bubble drag reduction in turbulent flow and opens the door for an optimization of the process.

\footnotetext{
${ }^{1}$ Published as: Ruben A. Verschoof, Roeland C.A. van der Veen, Chao Sun, and Detlef Lohse, Bubble drag reduction requires large bubbles, Phys. Rev. Let. 107, 104502 (2016).

Experiments by Verschoof and van der Veen. Data analysis by Verschoof. Verschoof and Lohse wrote the paper. Sun and Lohse supervised the project. All authors discussed the results and proofread the paper.
} 


\section{$5.1 \quad$ Introduction}

Theoretical, numerical and experimental studies on bubble drag reduction (DR) of a solid body moving in a turbulent flow have been performed for more than three decades [195, 213 217]. In the last decade from the maritime industry there was a renewed interest into this subject and into air lubrication, because other contributions to the total drag such as pressure drag and wave drag have already been optimized thanks to sophisticated vessel design [2]. A few volume percent $(\leq 4 \%)$ of bubbles can reduce the overall drag up to $40 \%$ and beyond $9,11,196,208,211,218$. However, the exact physics behind this drag reduction mechanism is unknown, thus hindering further progress and optimization, and even the dependence of the effect on the bubble size is controversial [205 207], though it is believed to be independent of the bubble size [217].

In this chapter, we experimentally investigated the mechanism behind bubble drag reduction in a Taylor-Couette (TC) system, i.e. the flow between two independently rotating coaxial cylinders. The TC system can be seen as "drosophila" of physics of fluids, with many concepts in fluid dynamics being tested therewith, ranging from instabilities, to pattern formation, to turbulence; see the reviews [50,51]. Here we inject bubbles into the system, which due to the density difference to water experience a centripetal force towards the inner cylinder, mimicking the upwards gravitational force acting on bubbles under a ship hull.

\subsection{Experiments}

The experiments are performed in the Twente Turbulent Taylor-Couette facility $\left(\mathrm{T}^{3} \mathrm{C}\right) 3$, with the inner one strongly rotating, corresponding to very large Reynolds number of $\operatorname{Re} \sim 10^{5}-10^{6}$. The setup has an inner cylinder with a radius of $r_{i}=200$ $\mathrm{mm}$ and an outer cylinder with a radius of $r_{o}=279 \mathrm{~mm}$, resulting in a radius ratio of $\eta=r_{i} / r_{o}=0.716$. The inner cylinder rotates with a frequency up to $f_{i}=20 \mathrm{~Hz}$, resulting in Reynolds numbers up to $\operatorname{Re}=2 \pi f_{i} r_{i}\left(r_{o}-r_{i}\right) / \nu_{\alpha}=2 \times 10^{6}$, in which $\nu_{\alpha}$ is the kinematic viscosity of water-bubble mixture. At these rotation rates, the influence of gravity is negligible compared to the centripetal acceleration [3]. The outer cylinder is at rest. The cylinders have a height of $L=927 \mathrm{~mm}$, resulting in an aspect ratio of $\Gamma=L /\left(r_{o}-r_{i}\right)=11.7$. The flow is cooled through both end plates to prevent viscous heating through the viscous dissipation. The torque $\tau$ is measured with a coaxial torque transducer (Honeywell Hollow Reaction Torque Sensor 2404-1K, maximum capacity of $115 \mathrm{Nm}$ ), mounted inside the middle section of the inner cylinder, to avoid measurement uncertainties due to seals and bearing friction and end plate effects. Details are described in Ref. [3. The gap between the cylinders is either fully filled with water $\left(T=20^{\circ} \mathrm{C}\right)$ or, when measuring with bubbles, partly filled with water. The effective viscosity and density of a bubbly liquid can be approximated using $\rho_{\alpha}=\rho(1-\alpha)$ and the Einstein relation 9, 204, $\nu_{\alpha}=\nu\left(1+\frac{5}{2} \alpha\right)$, in which $\rho$ and $\nu$ are the density and the viscosity of the single phase liquid, and $\alpha$ is the global 

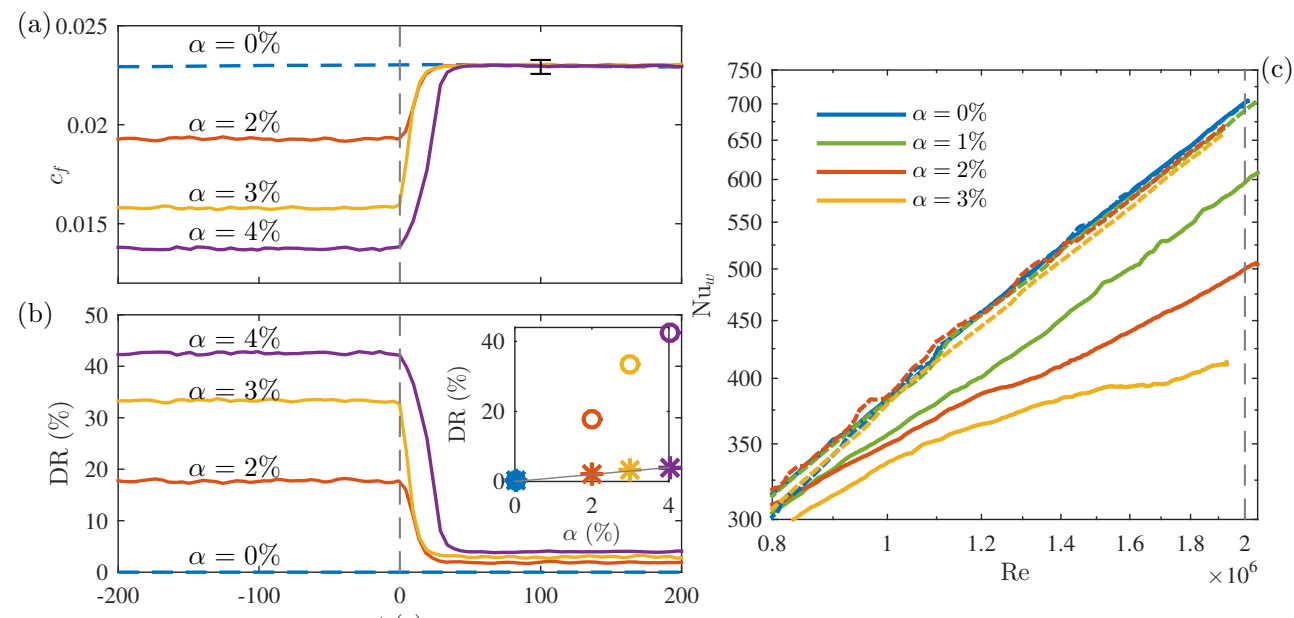

(b)

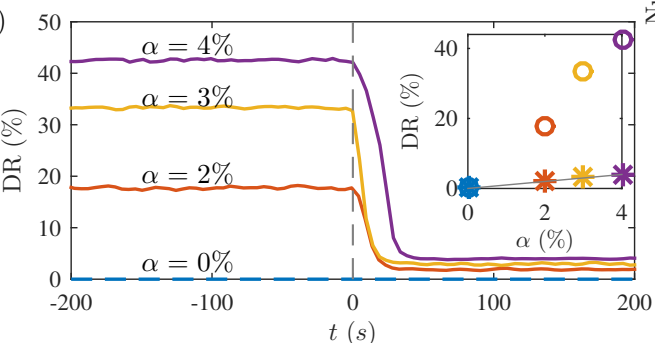

Figure 5.1: (a) Drag coefficient $c_{f}$ as a function of time (for $f_{i}=20 \mathrm{~Hz}$, corresponding to $\operatorname{Re}=2.0 \cdot 10^{6}$ at $\alpha=0 \%$ ) for different gas volume fractions $\alpha$. At $t=0 \mathrm{~s}$ the surfactant is injected, as indicated by the dashed vertical line. We then observe a large jump in the measured friction coefficient and within $\sim 20$ s all curves overlap. (b) Drag reduction (DR) as function of time. Nearly all DR is lost after injection of the surfactant at $t=0 \mathrm{~s}$. Inset: averaged DR before (circles) and after (asterisks) addition of the surfactant, as a function of the gas volume fraction $\alpha$. The thin line equals $\mathrm{DR}=\alpha$, showing that after addition of the surfactant the small residual DR is accounted for by the reduced density of the fluid mixture. (c) The dimensionless torque $\mathrm{Nu}_{\omega}=\tau / \tau_{\text {lam }}$, which is the torque $\tau$ divided by the torque in the laminar and purely azimuthal case [51], as a function of the Reynolds number Re for various $\alpha=$ $0 \%, 1 \%, 2 \%, 3 \%$, both with (dashed lines) and without (solid lines) the surfactant Triton X-100. (a),(b) correspond to $\operatorname{Re}=2.0 \cdot 10^{6}$ (at $\alpha=0 \%$ ), shown by the thin vertical line in the plot.

volume fraction of air. Air bubbles form over the entire cylinder height because of the large turbulent fluctuations and the high centripetal forces.

\subsection{Results and analysis}

The main result is seen in Figs. 5.1(a) and 5.1(b), where we show the drag coefficient $c_{f}(t)$ at $\mathrm{Re}=2 \times 10^{6}$ as function of time for four different bubble concentrations. It is calculated as $c_{f}=\tau /\left(L \rho_{\alpha} \nu_{\alpha}^{2} \operatorname{Re}^{2}\right)$ [see Fig. 5.1(a)] from the measured required torque $\tau(t)$ to keep the inner cylinder rotating at the fixed angular velocity $\omega_{i}$. While with bubble volume concentration between $2 \%$ and $4 \%$ the drag is remarkably reduced between $18 \%$ and $43 \%$ as compared to the single phase flow case without bubbles [9], adding the surfactant Triton X-100 at $t=0 \mathrm{~s}$ at a concentration of only $6 \mathrm{ppm}$ reduces the drag reduction within $20 \mathrm{~s}$ (the time needed for Triton $\mathrm{X}$ to mix over 
the whole system) to the value corresponding to the volumetric gas concentration of $2 \%-4 \%$. Here the percentage of drag reduction is expressed as $D R=\left(\tau^{\text {with }}\right.$ $\left.\tau^{\text {without }}\right) / \tau^{\text {without }}$, with the superscripts with and without referring to the cases with, and without surfactant, respectively. The same holds for weaker turbulence - here we tested down to $\mathrm{Re} \approx 8 \times 10^{5}$ [see Fig. 5.1(c)] — though for weaker turbulence the original drag reduction effect through the bubbles is less pronounced. Here, we show the dimensionless torque in terms of the Nusselt number $\mathrm{Nu}_{\omega}=\tau / \tau_{\text {lam }}$, in which $\tau_{\text {lam }}=4 \pi L \rho_{\alpha} \nu_{\alpha} r_{i}^{2} r_{o}^{2} \omega_{i} /\left(r_{o}^{2}-r_{i}^{2}\right)$ is the torque needed in the purely azimuthal and laminar flow case. The Nusselt number can also be directly related to the drag coefficient $c_{f}$ by $\mathrm{Nu}_{\omega}=c_{f} \omega_{i}\left(r_{o}-r_{i}\right)^{2}\left(r_{o}^{2}-r_{i}^{2}\right) /\left(4 \pi \nu_{\alpha} r_{o}^{2}\right)$.

Figure 5.2 shows snapshots of the bubbly turbulence at three different length scales (reflecting the multiscale character of bubbly turbulence) without (upper row) and with (lower row) the addition of Triton X-100. It is seen that the addition of the surfactant dramatically changes the structure of the turbulent dispersed bubbly flow, resulting in much smaller bubbles (with the same total volume concentration) in the case with Triton X-100. The reason is that the surfactant suppresses bubble coalescence 194,203]. Earlier studies noticed the role of the bubble Weber number in bubble drag reduction $9,11,214$. The Weber numbers We $=\rho_{\alpha} u^{\prime 2} D_{\text {bubble }} / \sigma$ before and after addition of Tritox X-100 are estimated as follows: From Fig. 5.2. we estimate that the equivalent bubble diameters are of order $D_{\text {bubble, without }}=O(1$ $\mathrm{mm}$ ) for clean water, and $D_{\text {bubble,with }}=O(0.1 \mathrm{~mm})$ for water with Triton X-100, respectively. The surface tension between water and air is known for clean water, i.e. $\sigma_{\text {without }}=73 \mathrm{mN} / \mathrm{m}$ at room temperatures. After the addition of $6 \mathrm{ppm}$ Triton X-100 (equivalent to $5 \times 10^{-5} \mathrm{~mol} / \mathrm{L}$ ), the surface tension lowers to $\sigma_{w i t h}=40 \mathrm{mN} / \mathrm{m} 202$. The velocity fluctuations, as well as bubble sizes and the spatial distribution, are impossible to measure in the bulk of the flow after the addition of surfactant, as the bubble flow is too dense to be optically accessible. We know that without bubbles, $u^{\prime} \approx 0.03 \omega_{i} r_{i}[9]$ in the bulk of the flow, and that this ratio is constant over a large range of Reynolds numbers, as long as the flow is fully turbulent [219]. Furthermore, it has been shown that this ratio does not change much after adding a few percent of mm-sized bubbles [9]. For a rotation rate of $20 \mathrm{~Hz}$, we calculate that $u^{\prime}=0.76 \mathrm{~m} / \mathrm{s}$. We assume that this is a reasonable measure for the fluctuations in our bubbly flow. For lower Reynolds numbers, the velocity fluctuations become smaller, resulting in lower Weber numbers. We note that for the smaller bubbles the accelerations due to turbulent fluctuations are much larger than the centripetal accelerations. Therefore no bubble clustering close to the inner cylinder occurs 9 .

From the figures we estimate the corresponding Weber numbers in the two cases as $\mathrm{We}_{\text {without }} \approx 10$ and $\mathrm{We}_{\text {with }} \approx 1$, implying that prior to injection of the surfactant the bubbles can deform [as indeed seen from Figs. 5.2(b) and 5.2(c)], whereas this is not possible after Triton X-100 was added [which is consistent with Figs. 5.2(e) and 5.2(f)]. As shown in Fig. 5.1(c), drag reduction is less pronounced at lower Reynolds numbers. The physical reason for this trend is that the Weber number of the bubbles decreases when reducing the Reynolds number. 

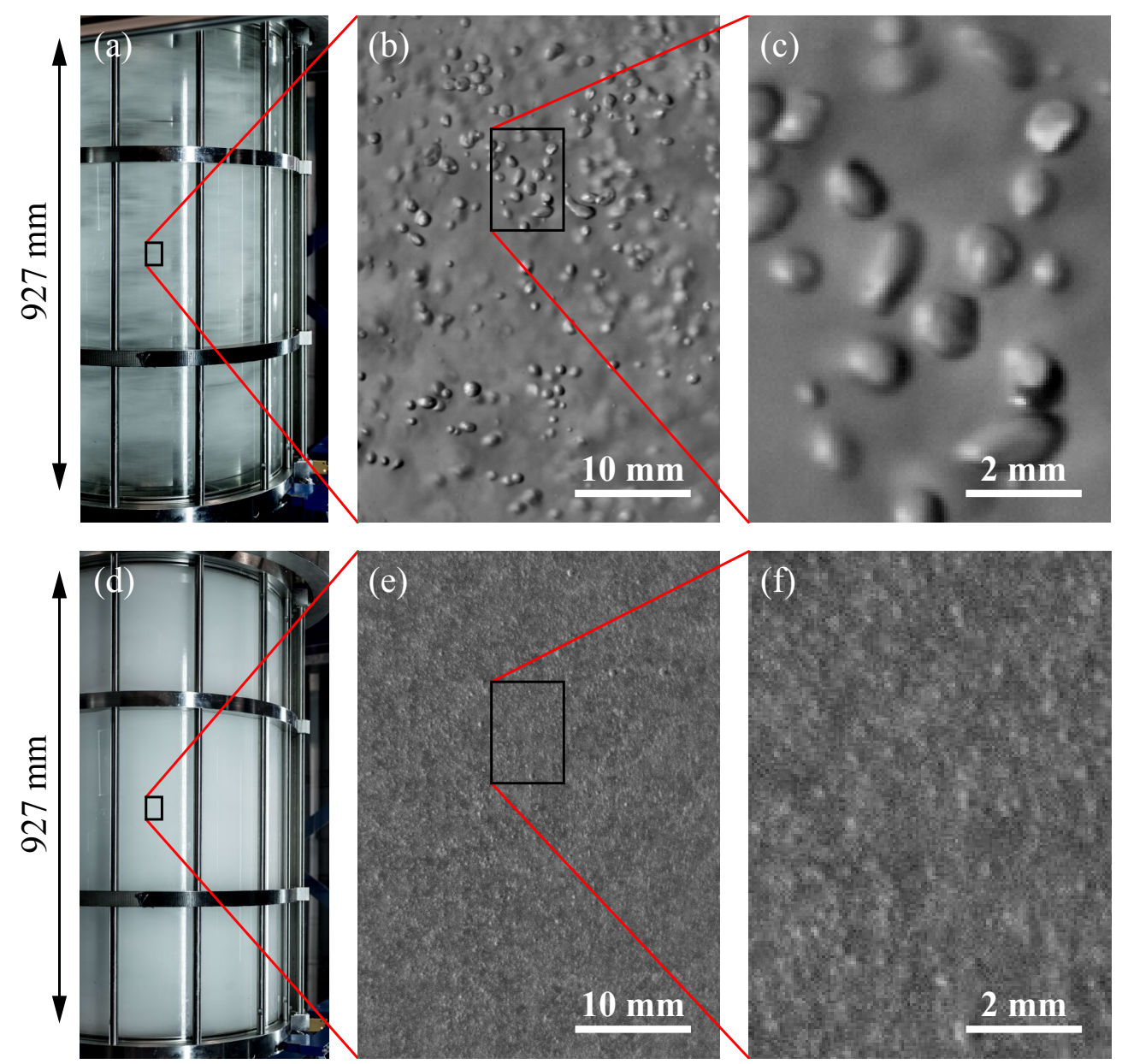

Figure 5.2: Snapshots of the bubbly turbulence $\left(\alpha=1 \%, \operatorname{Re}=2 \times 10^{6}\right)$ with increasing magnification (as shown by the scale bars). In the first row no surfactants are present in the turbulent flow, whereas the second row shows the (statistically stationary) situation after addition of $6 \mathrm{ppm}$ Triton $\mathrm{X}-100$. In the left photos the $\mathrm{T}^{3} \mathrm{C}$ apparatus can be seen. 


\subsection{Conclusions}

Our findings give strong evidence that the bubble deformability is crucial in the drag reduction mechanism, as already speculated in Refs. 9205210 , but disputed by other authors. We note that both the shape change of the bubble and the bubble coating by the surfactant will also modify the lift force coefficient of the lift acting on the bubble $194 \quad 197201203.208$ and thus the bubble distribution in the flow. Apparently, the large and deforming bubbles, which accumulate close to the inner cylinder [9], hinder the angular momentum exchange between boundary layer and bulk by partly blocking the emission of coherent structures from the boundary layer towards the bulk and reducing the Reynolds stress, thus leading to drag reduction [9, 193, 197, 205].

Our results have strong bearing on the projected bubble drag reduction in the naval industry. Not only surfactants, but also ions of the various dissolved salts have a strong effect on coalescence properties of bubbles, either enhancing or suppressing coalescence 198. As seen from our experiments, tests of bubbly drag reduction in fresh water facilities will therefore lead to very different results as in the salty ocean water.

Our results, however, also offer opportunities to enhance drag reduction in pipelines transporting liquified natural gases (LNGs) close to the boiling point by adding appropriate surfactants helping coalescence [200]. Going beyond bubbly multiphase flow towards emulsions of e.g. oil in water 199, also here the global drag will be strongly affected by the local coalescence behavior of the droplets, thus opening opportunities to influence the overall drag by the use of surfactants. 


\section{Chapter 6}

\section{Air cavities at the inner cylinder of turbulent Taylor-Couette flow!}

Air cavities, i.e. air layers developed behind cavitators, are seen as a promising drag reducing method in the maritime industry. Here we utilize the Taylor-Couette (TC) geometry, i.e. the flow between two concentric, independently rotating cylinders, to study the effect of air cavities in this closed setup, which is well-accessible for drag measurements and optical flow visualizations. We show that stable air cavities can be formed, and that the cavity size increases with Reynolds number and void fraction. The streamwise cavity length strongly depends on the axial position due to buoyancy forces acting on the air. Strong secondary flows, which are introduced by a counterrotating outer cylinder, clearly decrease the stability of the cavities, as air is captured in the Taylor rolls rather than in the cavity. Surprisingly, we observed that local air injection is not necessary to sustain the air cavities; as long as air is present in the system it is found to be captured in the cavity. We show that the drag is decreased significantly as compared to the case without air, but with the geometric modifications imposed on the TC system by the cavitators. As the void fraction increases, the drag of the system is decreased. However, the cavitators itself significantly increase the drag due to their hydrodynamic resistance (pressure drag): In fact, a net drag increase is found when compared to the standard smooth-wall TC case. Therefore, one must first overcome the added drag created by the cavitators before one obtains a net drag reduction.

\footnotetext{
${ }^{1}$ Ruben A. Verschoof, Dennis Bakhuis, Pim A. Bullee, Sander G. Huisman, Chao Sun, and Detlef Lohse, Air cavities at the inner cylinder of turbulent Taylor-Couette flow, accepted for publication at Int. J. of Multiphase Flow.

Experiments by Verschoof, Bakhuis and Bullee. Verschoof analysed the data and wrote the paper. Huisman, Sun and Lohse supervised the project. All authors discussed the results and proofread the paper.
} 


\subsection{Introduction}

Around $90 \%$ of the world trade is carried by cargo vessels. Therefore, even a minor energy saving in this industry has a major impact on global fuel savings and $\mathrm{CO}_{2}$ emissions. One method to save the overall fuel consumption is to reduce the resistance between the hull and the surrounding water. Next to wave drag and pressure drag (or form drag), viscous skin friction is the major contribution to the total friction, accounting for approximately half of the total resistance [143]. Wave drag and pressure drag can be optimized by a careful design of the shape of the vessel. Skin friction, however, cannot be optimized similarly, as it is proportional to the wetted area of the hull [2].

One of the most promising techniques in naval engineering to reduce the skin friction is the use of air lubrication. Air lubrication can be applied in the form of bubbly drag reduction (DR) or — presumably more effective — in the form of air layer drag reduction 217. Both methods have been studied, mainly experimentally, in great detail over a wide range of Reynolds numbers and bubble diameters, mostly in water channels and flat plate configurations, see e.g. the review articles [214,217]. The mechanism of bubbly DR is not yet entirely known, but it is clear that large, deformable bubbles are effective in reducing the friction in the boundary layer 9 , 126 205. The working principle of DR using air layer is more intuitive: An air layer prevents the water from contacting the hull, thus decreasing the wetted area. The DR can be over $80 \%$, as reported in several studies, as long as the air layer is stable and well-developed 139,208,209,218.

Air layers are formed when sufficient amount of air is injected under a vessel or flat plate 209, 218. Although it is a straightforward technique, its drawbacks are the low stability of the air layer and excessive necessary air injection rates, see e.g. ref. 134 for a recent literature overview of air layers and air discharge mechanisms.

One way of improvement is by installing a raised edge, commonly referred to as the "cavitator", see figures 6.1 and 6.2 for typical cavitator shapes. The cavitator creates a region of low pressure at the leeside which stimulates air to attach to the wake of the cavitator, thus decreasing the necessary air injection rate and increasing stability. This air layer developed downstream of the cavitator is called "air cavity". Air is usually injected directly at the cavitator, attaches to it, and forms the cavity. Eventually, the air is discharged from the air cavity in the "closure region", thus forming a contact line at the wall-water-air interface. In this region, the drag is increased due to local cavity shedding and re-entrant flows [217. The streamwise air cavity length was found to depend on the gravity wavelength $\lambda$, which is found through the dispersion relation $u_{\infty}=\sqrt{\frac{g \lambda}{2 \pi} \tanh \left(\frac{2 \pi D}{\lambda}\right)}$, in which $D$ is the water depth, $u_{\infty}$ the free-stream velocity and $g$ the gravitational acceleration $130-132$. The maximum stable air cavity length then equals half the gravity wavelength $\lambda$, which thus only depends on the water depth and vessel speed . Experiments indeed confirmed that the cavity length is virtually independent on the cavitator height and air injection rate [134]. Two different air discharge mechanisms are known in the closure region: 
i) wave pinch-off and ii) a re-entry jet 217]. The wave pinch-off mechanism is related to interfacial waves, that can pinch off patches of air when the air cavity is thin. The re-entry jet is formed by a stagnation point in the wake of the cavity. This jet flows upstream, leading to a periodic break-off of air 140. Note that the nomenclature of the "cavitator" is unrelated to real cavitation, i.e. the rapid liquid-to-vapor phase transition.

When applying air cavities, a significant difference between net and gross DR is present. A compressor to blow air under the hull consumes energy, and locally the drag is increased in the closure region and by the cavitators and skegs, which are installed to prevent air from discharging sideways. In fact, the challenge is not to increase the drag by the geometric changes, but to find a net drag reduction. Therefore one has to find the optimum between DR and additional energy losses and also expenses. Recent full-scale ship measurements resulted in impressive net power savings of $10 \%$ to $20 \%$ 133, $135,138,213$. However, despite its clear potential advantages, air lubrication is hitherto not widely used. One of the main issues it that laboratory results are hard to scale up to the conditions of real applications and therefore the performance of ships is difficult to predict [214]. Although efforts are being made to numerically model air cavities, presently only RANS and LES simulations are common, and thus closure models, which are not always reliable, are necessary 113. In this field of research, many questions remain unanswered, and there is a clear need for well-controlled, precise measurements to study the underlying physics.

The goal of this study is to explore the possibilities of studying air cavities in Taylor-Couette (TC) flow. Taylor-Couette flow, i.e. the flow between two concentric, independently rotating cylinders, is one of the canonical systems in which fluid flow physics is studied, see the recent reviews refs. 50, 51, and fig. 6.1 for a schematic of a TC setup. It has the advantage of being a closed system with an exact balance between driving and energy dissipation, and it is accessible experimentally thanks to its simple geometry. Furthermore, it is a compact system in which highly turbulent flows can be studied. By using a TC apparatus, we have the opportunity to study air cavities in a highly controlled environment.

In TC flow, the driving of the flow is expressed through two Reynolds numbers, namely $\operatorname{Re}_{i}=\omega_{i} r_{i}\left(r_{o}-r_{i}\right) / \nu$ for the inner cylinder and $\operatorname{Re}_{o}=\omega_{o} r_{o}\left(r_{o}-r_{i}\right) / \nu$ for the outer cylinder. Here, $r_{i}$ and $r_{o}$ are the radii of the inner and outer cylinder, respectively, $\omega_{i}$ and $\omega_{o}$ are the angular velocities of the inner and outer cylinder, respectively, and $\nu$ is the kinematic viscosity. The primary response parameter is the torque $\tau$ necessary to rotate the cylinders at a constant driving speed, i.e. at constant $\operatorname{Re}_{i}$ and $\operatorname{Re}_{o}$. The torque is made dimensionless as $G=\tau /\left(2 \pi L_{I C} \rho \nu^{2}\right)$, in which $\rho$ is the fluid density and $L_{I C}$ is the height of the inner cylinder, i.e. the height over which the torque is measured.

Taylor-Couette flow has been used extensively to study bubbly drag reduction and bubble dynamics, see e.g. refs. $9,11,97,115,117,126,141,142,177,210$. At lower Reynolds numbers, small bubbles do not only beautifully visualize Taylor rolls and other vortical structures in the flow [114, they also decrease the drag by destroying 
the momentum transport in these vortices 112. At higher Reynolds numbers, in which the drag and shear rates are high, a small percentage of large bubbles has a tremendous effect on the global drag, e.g. at $\mathrm{Re}_{i}=2 \times 10^{6}$, DR percentages of $40 \%$ were observed for a global gas volume fraction of only $4 \%[9]$. These DR percentages reach far beyond the trivial effects of the changed effective density and viscosity. Efforts are being made to study thin air layers around the inner cylinder in TC flow, which can be achieved by using a superhydrophobic coating [128,129], or by heating the inner cylinder, thus creating a Leidenfrost vapour layer [127]. Studying air cavities in TC flow has, to the best of our knowledge, never been done before.

The outline of this chapter is as follows. First, we describe the experimental method in section 6.2. In section 6.3.1 we show the existence of air cavities by showing and interpreting high-speed recordings. From these visualizations, we extract the cavity length and global coverage in section 6.3.2 We continue by presenting the torque measurements and resulting drag reduction in section 6.3.3, thus quantifying the flow behaviour. We conclude this study in section 6.4.

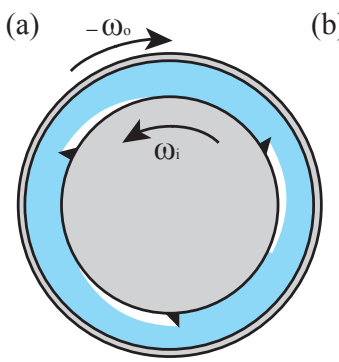

(b)

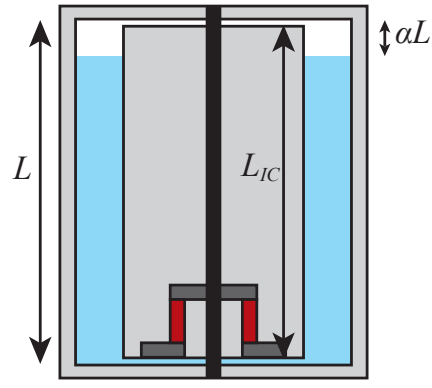

Figure 6.1: Experimental setup. (a) Top view schematic of the $\mathrm{T}^{3} \mathrm{C}$ facility (not to scale). Air is captured at the leeward side of the cavitators, as indicated. We attached 2,3 , or 6 cavitators equally distributed around the perimeter of the inner cylinder. The rotation of the cylinders is shown as $\omega_{i}$ and $-\omega_{o}$. (b) Vertical crosssection, showing the position of the torque sensor. The sensor is located in the inner cylinder, so that the torque between the driving shaft and the inner cylinder is measured. To control the void fraction, we fill the cylinder only partially with water, so that the void fraction $\alpha$ is controlled by measuring the relative height of the water level. Turbulent mixing ensures axial mixing between the two phases, whereas the centrifugal accelerations push the water towards the outer cylinder and, consequently, the air towards the inner cylinder.

\subsection{Experimental method}

The experiments were performed in the Twente Turbulent Taylor-Couette $\left(\mathrm{T}^{3} \mathrm{C}\right)$ facility, see ref. 4 for all technical details. The setup has inner and outer cylinder radii of $r_{i}=200 \mathrm{~mm}$ and $r_{o}=279.4 \mathrm{~mm}$, respectively, giving a radius ratio of 
$\eta=r_{i} / r_{o}=0.716$ and a gap width $d=r_{o}-r_{i}=79.4 \mathrm{~mm}$. The maximum rotationfrequencies of the inner and outer cylinder with cavitators are $f_{i}=10 \mathrm{~Hz}$ and $f_{o}= \pm 5$ $\mathrm{Hz}$, respectively, resulting in Reynolds numbers up to $\mathrm{Re}_{i}=2 \pi f_{i} r_{i} d / \nu=1 \times 10^{6}$ and $\operatorname{Re}_{o}= \pm 2 \pi f_{o} r_{o} d / \nu= \pm 7 \times 10^{5}$ with water as the working fluid with a temperature of $T=20 \pm 0.5^{\circ} \mathrm{C}$. We continuously measure the temperature, and use the instantaneous temperature-dependent viscosity and density to calculate our dimensionless quantities. The rotation ratio between outer and inner cylinder is defined as $a=-f_{o} / f_{i}$. When both cylinders rotate, we express the driving as a "shear Reynolds number", i.e.

$$
\operatorname{Re}_{s}=\frac{r_{i}\left(\omega_{i}-\omega_{o}\right) d}{\nu}=\operatorname{Re}_{i}-\eta \operatorname{Re}_{o}
$$

in which $\omega_{i, o}=2 \pi f_{i, o}$. In this study, the rotation rates are limited by vibrations in

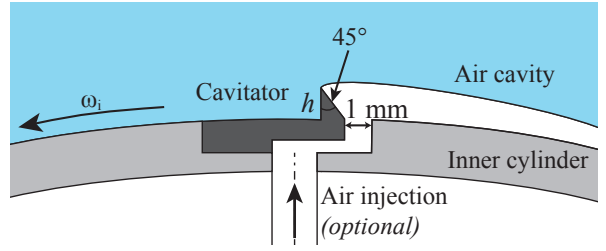

Figure 6.2: Sketch of the cavitator and air injector. The cavitator, here shown in dark gray extends over the entire height of the inner cylinder, see also fig. 6.3. The cavitator edge has a sharp, $45^{\circ}$ corner, with a height of $h=2 \mathrm{~mm}$. This sharp edge, although introducing pressure drag, is necessary to start a stable air layer. Using a rotary union-slip ring combination, air is led through the shaft, meaning that air can be injected through the inner cylinder while the cylinder is rotating. This way of active air injection is optional.

the system, which are caused by the uneven distribution of air. The outer cylinder has a height of $L=932 \mathrm{~mm}$, giving an aspect ratio of $\Gamma=L / d=11.7$. The inner cylinder has a height of $L_{I C}=927 \mathrm{~mm}$. The transparent acrylic outer cylinder allows for flow visualizations. The end plates, which are partly transparent, are fixed to the outer cylinder. The torque $\tau$ is measured with a co-axial torque transducer (Honeywell 2404-1K), placed inside the inner cylinder to avoid measurement errors caused by seal- and bearing friction, see fig. 6.1. All flow visualizations are made with a Photron FASTCAM SA-X high-speed camera with a resolution of $1024 \times 1024$ px. As illumination we used a Briteq BT-Theatre-1EZ LED theatre spotlight. We stress that a uniform light intensity is hard to achieve due to the curved surface of the cylinders. A Zeiss Makro $50 \mathrm{~mm}$ lens was used, resulting in a field of view of $23^{\circ}$ in both the horizontal and vertical direction. We fix 2,3 , or 6 cavitators to the inner cylinder (IC), as shown in figures 6.1 and 6.2. Due to a local low pressure, air attaches to it in the wake of the cavitator. The cavitators extend over almost the entire height of the cylinders, and have a height of $2 \mathrm{~mm}$. Shape and size effects of the cavitator have been studied in a water tunnel configuration [134], indicating that a sharp cavitator tip is necessary. 


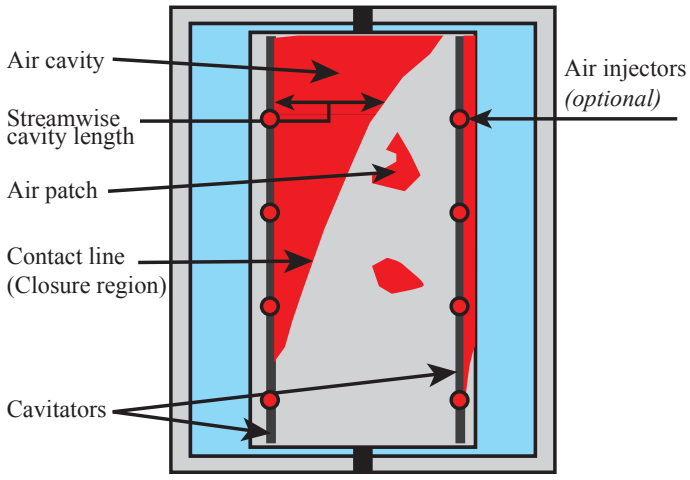

Figure 6.3: A sketch of the front view of the setup with a developed air cavity, which is shown in red to ease readability. The cavitator is shown in dark gray. We indicate the position of all relevant features encountered in this study. The height of the air injectors equals $z / L=0.09,0.33,0.57,0.81$.

The amount of air is characterised by the global void fraction $\alpha$. In the current study, we used 2 procedures of controlling the amount of air in the setup: i) We do fill the apparatus only partially with water, leaving room for a controlled amount of air, which is measured with both cylinders at rest. Already at the lowest Reynolds numbers presented here, turbulent mixing ensures distribution of air in the bulk flow (see fig. 6.1 and e.g. 126]). This procedure is used, unless mentioned otherwise. Or ii) We actively inject air from the cavitators at four axially distributed heights, as indicated in figs. 6.2 and 6.3 . An overflow channel allows air to leave the setup and prevents pressure build-up in the system. After each measurement, when both the cylinders and the fluid are at rest again, we remeasure the void fraction, to ensure that the void fraction was constant during the measurement. With this procedure, the uncertainty in void fraction is kept below $\alpha_{\text {err }} \leq 0.1 \%$. We note that in both procedures, the local effective local void fraction depends on axial and radial position due to buoyancy and centripetal forces, as shown in [9].

\subsection{Results}

\subsubsection{Flow visualizations}

The goal of these visualizations is two-fold: i) we can qualitatively study the flow dynamics to prove the existence of air cavities in TC flow, and ii), we can extract the streamwise air cavity length and air coverage from these images. We first visualize the entire TC setup, as shown in fig. 6.4. The air cavity is best visible in fig. 6.4. Here, we see the cavity over a considerable portion of the cylinder, especially in the top part of the setup. We observe the interfacial capillary waves at the surface of the air cavity, and we see bubbles or air patches at locations where the cavity is not formed. See the annotations in fig. 6.3 and 6.4 , in which all relevant flow features are 


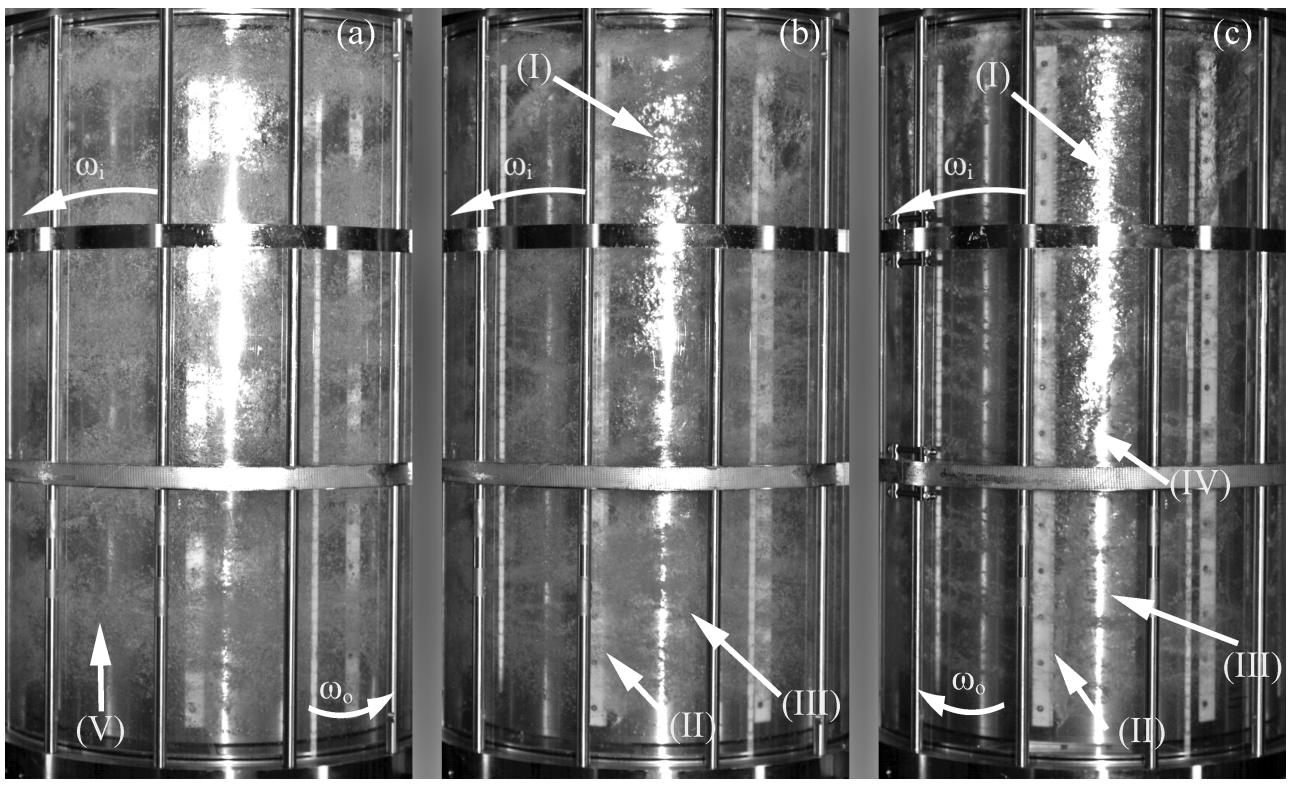

Figure 6.4: Snapshots of air cavities at $\operatorname{Re}_{s}=8 \times 10^{5}$, for 3 different rotation ratios: (a) $a=0.14$ (counter-rotation), (b) $a=0$ (stationary OC) and (c) $a=-0.2$ (corotation). The direction of the cylinder rotation is indicated by the curved arrows, in which $\omega_{i}$ and $\omega_{o}$ indicate the direction of the inner and outer cylinder, respectively. The global gas volume fraction is $\alpha=2 \%$. The vertical bars and horizontal rings are essential structural parts of the setup. (I) The air cavity. (II) The cavitator. (III) Cylinder not covered with an air cavity. (IV) The contact line of the cylinder-waterair interface. (V) In the counter-rotating case, many bubbles are trapped in these 'Taylor vortices'. In fig. (b) and especially in fig. (c), the flow is radially more stably stratified. Therefore, less bubbles are present and the air cavity is better visible.

highlighted.

In fig. 6.4 the cylinders counterrotate, which is known to induce turbulent Taylor vortices [5, 176]. These vortices are visualized here by the bubbles captured within them. The vortices, which introduce strong secondary flows in the system, prevent the bubbles from sticking to the inner wall. Apparently, the air cavity is largely destabilized and destroyed in the counterrotating regime. In fig. 6.4 c, the opposite is the case. Corotating cylinders stabilise the flow and suppress secondary flows. We see that air is pushed towards the inner cylinder more effectively than in the counterrotating case. Fig. 6.4 b, in which only the inner cylinder rotates, shows an intermediate behaviour, i.e. no pronounced Taylor vortices, but nonetheless larger vortical structures in which bubbles are entrained.

To capture the local dynamics we zoom in, as shown in fig. 6.5. Here, we only rotate the inner cylinder. We clearly see the interfacial waves at the air-water interface. Also, it is clear that the majority of the air is indeed captured in the air cavity 

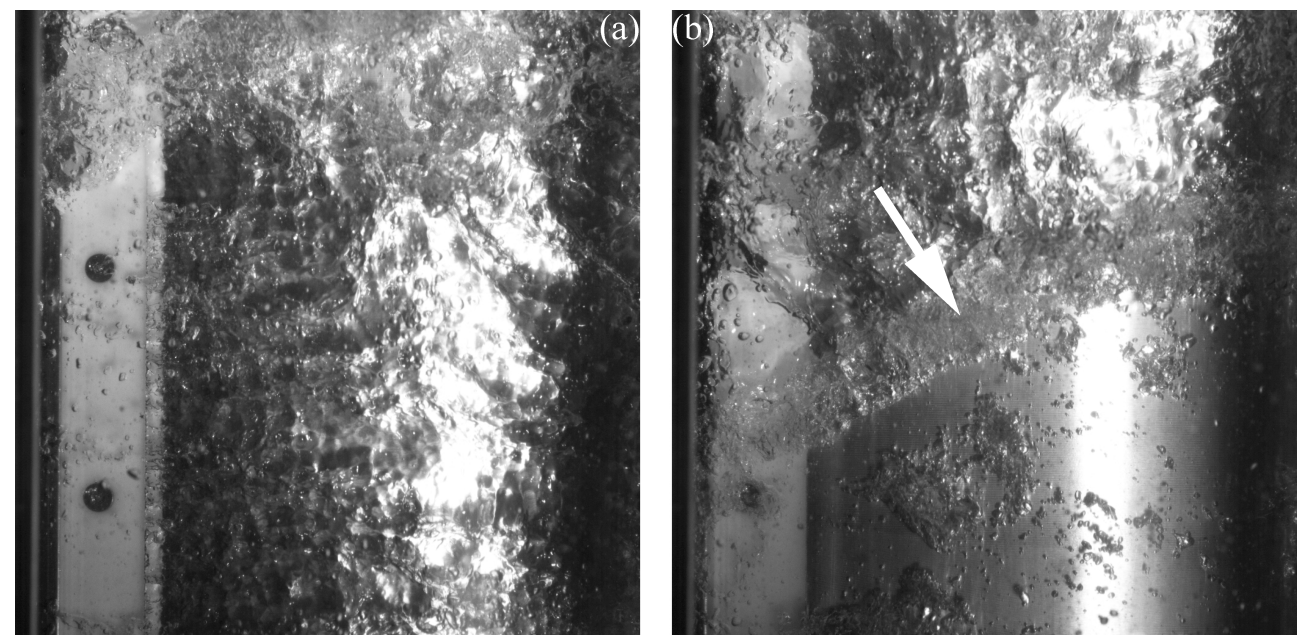

Figure 6.5: Snapshots of air cavities at $\operatorname{Re}_{i}=5 \times 10^{5}$ with a stationary outer cylinder, 3 cavitators, and $2 \%$ of air. We zoomed in on the top of the cylinder. We show 2 photos taken at time $=t_{1}$ - when the cavitator is visible, and $t_{2}$ - when the closure region is visible. (a) Cavitator (vertical white strip in image) with development of the air cavity at $t_{1}$. (b) The closure region of the air cavity at $t_{2}$. The visible white bar here is not a cavitator, but a blank that is mounted flush with the cylinder surface. Note the dependence of the cavity length on the height. The white arrow indicates the position of the closure region, which is governed by the re-entry jet mechanism.

- only few bubbles are present in the bulk. In the closure region, we see that the dominant breakup process is the "re-entry jet". This is clear from the local structure, which is more "blurry" than most of the cavity, as indicated by the white arrow in fig. 6.5 p.

\subsubsection{Cavity length and coverage}

The streamwise cavity length and global coverage are among the crucial parameters of the cavity as they govern the de-wetted area and thus the possible drag reduction. From images as shown in fig. 6.4, we extract the air cavity length. We first averaged 100 independent instantaneous photos of the flow, to get a time-averaged cavity length rather than the instantaneous value. Then, we manually tracked the edges of the cavity and the cavitator, and binarized the image, in which the area covered by cavity is distinguished from the area which is not. From this binarized image, we can extract the global coverage and the streamwise air cavity length at any axial position. Due to the structural parts blocking the view and light reflections, an automated procedure to extract the position of the air cavity turned out to be unfeasible. We do this for all cases with 3 cavitators and a stationary outer cylinder. Here, the cavity length is shown for 3 axial heights in fig. 6.6. The axial dependence, which already was made clear from fig. 6.4 and 6.5 is significant. Clearly, due to buoyancy forces 


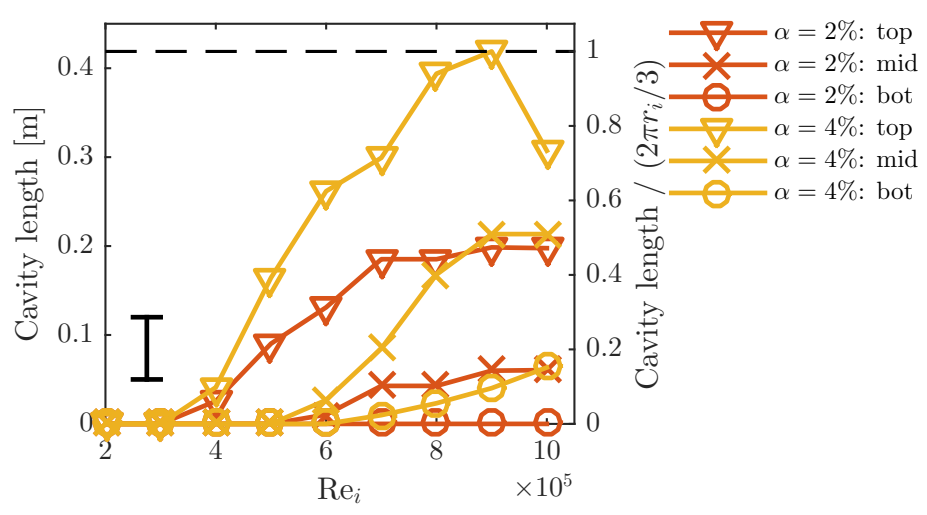

Figure 6.6: Streamwise air cavity length on the inner cylinder as a function of $\operatorname{Re}_{i}$. The outer cylinder is stationary. We used 3 cavitators. The coverage is extracted by visual means from a series of images similar to those of fig. 6.4. We show results for three different axial positions, close to the top $(z / L=3 / 4)$, at mid-height $(z / L=1 / 2)$ and close to the bottom $(z / L=1 / 4)$. The estimated error bar is shown in the bottom left corner of the graph. In dashed black, we added the streamwise length between two cavitators $2 \pi r_{i} / 3$, which is the upper limit of the streamwise cavity length. On the right $\mathrm{y}$-axis, we normalized the streamwise cavity length with the distance between two cavitators.

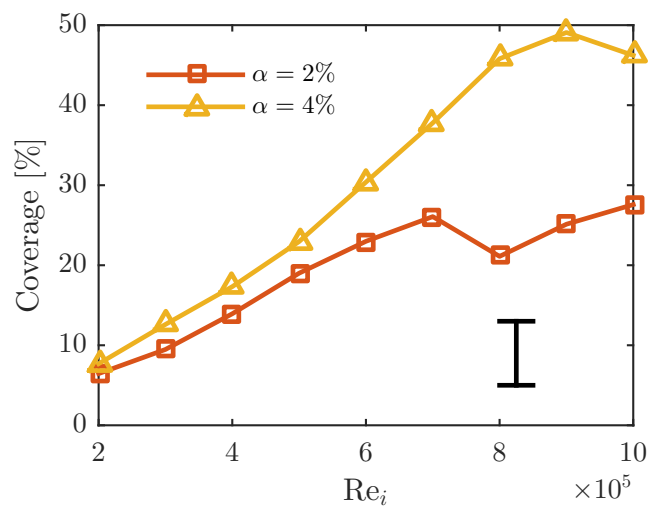

Figure 6.7: Percentage of air cavity coverage on the inner cylinder as a function of $\operatorname{Re}_{i}$. The outer cylinder is stationary. We used 3 cavitators. The coverage is calculated by integrating the streamwise cavity lengths (see fig. 6.6). The estimated error is shown in the bottom right corner of the graph. 
air has an spatial preference towards the top of the cylinders. Previous bubble DR measurements already showed the axial dependence of the location of bubbles, even when air is continuously injected from the bottom 9]. We see here that this axial dependence is present also in the case of air cavities. We note that although the cavity length at the three shown axial heights for the smallest Reynolds numbers is zero, a small cavity already forms closer to the top.

The centrifugal acceleration at the inner cylinder is given by $a_{c e n t r}=\omega_{i}^{2} r_{i}$. Consequently, in the limit of $\mathrm{Re}_{i} \rightarrow \infty$, the gravitational forces are negligible as compared to $a_{c e n t r}$, and the axial dependence of the air cavity length will disappear. In the hypothetical case of air in a purely laminar TC flow, all air would be pushed towards the inner cylinder due to a radial pressure gradient caused by the centrifugal forces [9]. In this turbulent flow, however, strong velocity fluctuations are present, causing the air to distribute itself over the entire gap width, even though the preferential accumulation close to the inner wall remains present 9 . The number of cavitators does not influence the cavity length, as long as the air cavities can be regarded as isolated [134]. If the cavitators are so closely spaced that cavitators are within range of an upstream cavity, they become submerged in air and a continuous air cavity is formed.

We calculate the air coverage for both $\alpha=2 \%$ and $\alpha=4 \%$, to know whether the global gas fraction influences the coverage, see fig. 6.7. The coverage is calculated by integration of the streamwise cavity lengths over the entire height of the cylinder, which is then divided by the total area between 2 cavitators (i.e. $2 \pi r_{i} L_{I C} / 3$ ). We see that the coverage for both measured gas fractions are similar up to $\operatorname{Re}_{i}=6 \times 10^{5}$, after which the $\alpha=2 \%$-curve saturates at a coverage of $25 \%$. The coverage for $\alpha=4 \%$ increases up to $\operatorname{Re}_{i}=8 \times 10^{5}$, where it saturates at a coverage of $45 \%$.

In these 2 saturation coverages regimes, the majority of the air is attached to the cavity, and not dispersed throughout the flow as bubbles. Assuming that all air is attached to the cavity, it is possible to get an estimate of the thickness of the air cavity. The surface area covered of the inner cylinder equals $2 \pi r_{i} L_{I C} \cdot$ coverage. We divide the volume of air in the setup, which equals $\alpha V=\alpha \cdot L_{I C} \pi\left(r_{o}^{2}-r_{i}^{2}\right)$, by the coverage surface area, to get a nominal value for the thickness $h_{\text {cavity }}$. For both $\alpha=2 \%$ and $\alpha=4 \%$ we find that $h_{\text {cavity }} \approx 8 \mathrm{~mm}$, which is in line with earlier measurements [134. Note that the estimated $h_{\text {cavity }}$ is much larger than the cavitator height, which is $h=2 \mathrm{~mm}$.

Clearly, achieving a higher coverage would be beneficial, and could be achieved by measuring with a larger void fraction. Measurements at higher gas volume fractions are impossible due to vibrations of the system, which are caused by the uneven distribution of air.

\subsubsection{Torque and drag reduction}

Up to now, we focussed on flow visualizations and results which can be extracted from these. Now, we turn to torque measurements to quantify how the torque is affected by the air cavities. We study the influence of the Reynolds number, the void fraction $\alpha$, the number of cavitators and the effect of outer cylinder rotation on the global torque 
and drag reduction. In all measurements, we quasi-statically increase the rotation rates of the cylinders, and constantly measure the rotation rate, the torque, and the temperature in the flow. From the temperature we calculate the instantaneous viscosity and density. The drag reduction is defined as DR $=1-G(\alpha) / G_{0}$. Here, we use as $G_{0}=G(\alpha=0)$ the case without air, but with cavitators. For the calculation of $G$ we used the density and viscosity of water, and we did not corrected for any changed effective flow properties caused by the air.
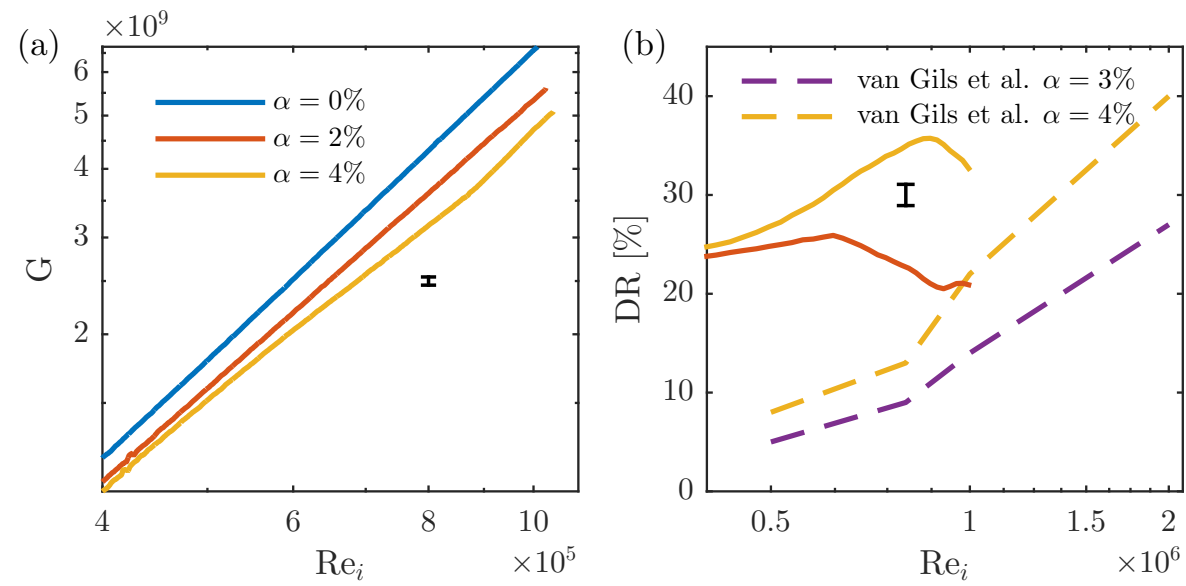

Figure 6.8: Global dimensionless torque and drag reduction percentage for $0 \%, 2 \%$, and $4 \%$ of air. Here we mounted 3 cavitators, and we kept the outer cylinder stationary. (a) Dimensionless torque $G$ as a function of the inner Reynolds number $\mathrm{Re}_{i}$. (b) Drag reduction percentages as a function of inner Reynolds number $\mathrm{Re}_{i}$. As comparison, we also show the bubbly DR results from 9 . A typical error bar is shown in both graphs.

We show results in fig. 6.8 Here, we mount 3 cavitators to the IC, and while keeping $\alpha$ constant, we quasi-statically increase $\omega_{i}$, and thus $\operatorname{Re}_{i}$. The outer cylinder is kept stationary. We clearly see that the dimensionless torque $G$ decreases with the presence of air cavities. When we compare our air cavity results with earlier measurements with bubbly DR, we clearly observe that air cavities decrease the drag more effectively for the same Reynolds number. Nevertheless, we see that the air cavity DR saturates from $\operatorname{Re}_{i}=8 \times 10^{5}$ and onwards, whereas the bubbly DR increases with increasing Reynolds number. This can be explained as follows. When applying air cavities, the DR largely depends on the coverage, which is shown in fig. 6.7. We see in fig. 6.7 that the air coverage saturates, which is reflected in the observed saturating DR. In fact, the shapes of fig. 6.7 and 6.8 b are similar. The relation between these two quantities is better revealed when plotting the DR as a function of air cavity coverage, see fig. 6.9. In bubbly DR, the bubbles do not necessarily attach to the cylinder. For bubbly DR, the relevant parameter is the Weber number, which is a measure for bubble deformability [9]. The Weber number increases with increasing Reynolds 


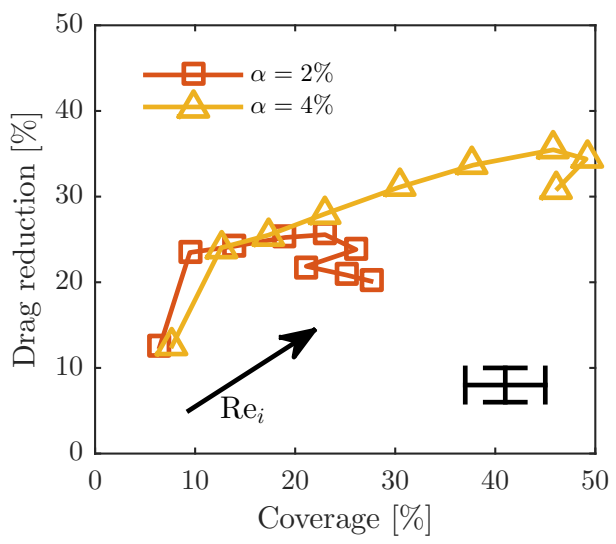

Figure 6.9: The drag reduction, as shown in fig. 6.8 as a function of air cavity coverage (fig 6.7). Note that both the $\mathrm{DR}$ and the coverage depend on $\mathrm{Re}_{i}$. A typical error bar is shown for both the drag reduction as the coverage percentage.
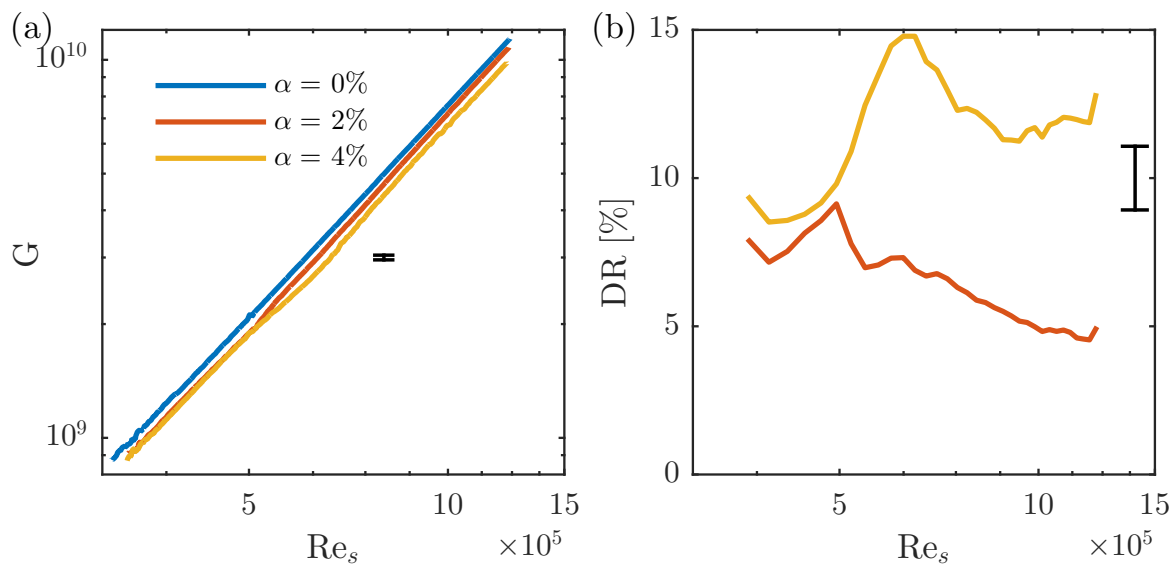

Figure 6.10: Dimensionless torque and DR for the case of counter-rotating cylinders with 3 cavitators as a function of shear Reynolds number $\operatorname{Re}_{s}$. The rotation ratio equals $a=0.2$. (a) Dimensionless torque $G$ as a function of shear Reynolds number $R_{s}$. (b) Drag reduction percentages as a function of shear Reynolds number $\operatorname{Re}_{s}$. The DR is significantly smaller than for the case of only inner cylinder rotation (fig. 6.8. A typical error bar is shown in both graphs. 
number, hence the increasing DR. In the present study, the mechanism of DR is different, and as the coverage is limited by the void fraction, the DR consequently is limited too.

In the $\mathrm{T}^{3} \mathrm{C}$ setup, we have the possibility to rotate the outer cylinder. It is known that for single-phase TC flow, the counterrotating cylinders enhance secondary flows, i.e. turbulent Taylor vortices 5, 51, 176]. These Taylor vortices enhance the momentum transport from inner to outer cylinder, thus increasing the global torque 4]. By measuring in the counter-rotating regime we can study the influence of strong secondary flows on the cavity and the drag reduction. We are not aware of any prior measurements of bubbles in turbulent TC flow with counterrotating cylinders. In these experiments we fix the rotation ratio between outer and inner cylinder to a rotation rate $a=-f_{o} / f_{i}=0.2$. The results are shown in fig. 6.10. Also in the counter-rotating regime the drag is decreased by air cavities. However, the DR is smaller than for pure inner cylinder rotation. This can be explained as follows. In fig. 6.4 we see that many bubbles are entrapped in the turbulent Taylor vortices, as the strong radial flow drags air away from the inner cylinder. Therefore, strong secondary flows decrease the stability of the air cavity, thus suppressing drag reduction.
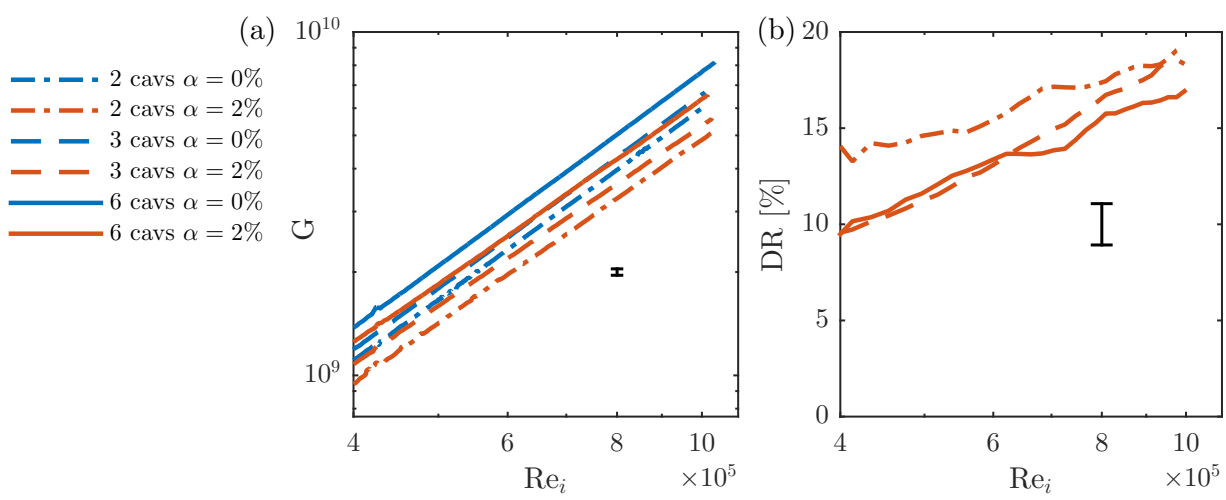

Figure 6.11: (a) Dimensionless torque with 2, 3, or 6 cavitators as a function of $\operatorname{Re}_{i}$ for stationary outer cylinder. (b) The drag reduction for $\alpha=2 \%$ for the case with 2,3 or 6 cavitators. The DR percentages are similar for a constant gas volume fraction $\alpha$, although the global torque is increased by the cavitators, which induce an additional pressure drag 220]. A typical error bar is shown in both graphs.

We now vary the number of cavitators. We installed 2, 3, or 6 cavitators, and while keeping the outer cylinder stationary we measured the torque for void fractions of $\alpha=0 \%$ and $\alpha=2 \%$. The DR here is calculated comparing the case with $\alpha=2 \%$ to the single-phase water case for the same number of cavitators. The results, which are shown in fig. 6.11, show that the DR is very similar. However, the absolute torque values clearly differ, and increase with the number of riblets. E.g. here we see that the torque with 6 cavitators and $2 \%$ void fraction is larger than the torque with 2 cavitators case without air. Since we clearly observe the additional drag caused by 
the cavitators, it is crucial to study the effect of the pressure drag at the cavitators in more detail.
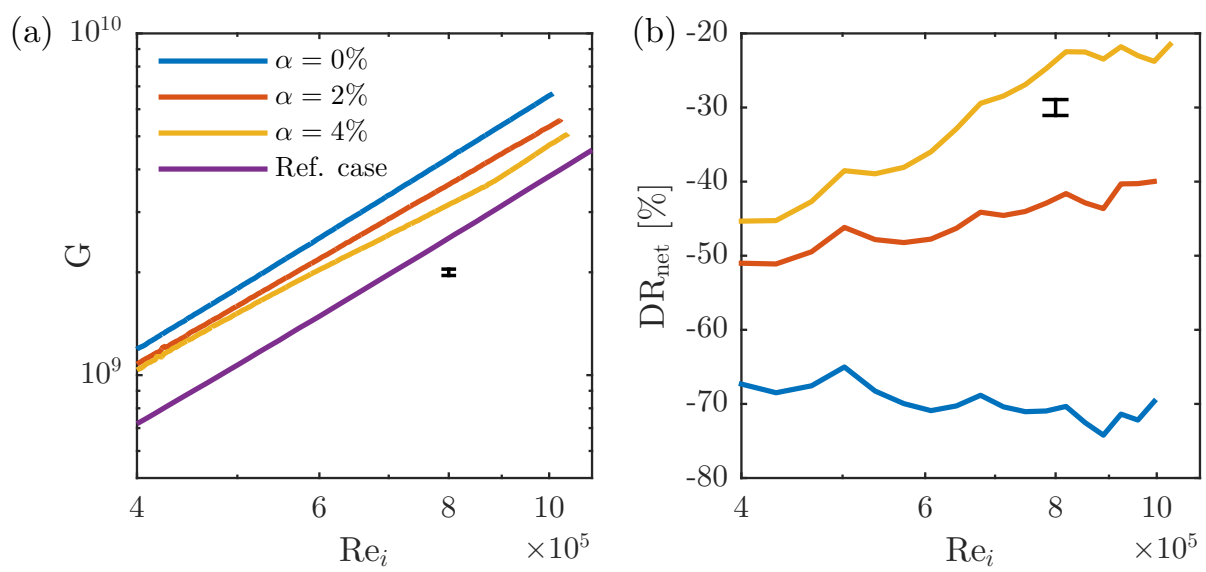

Figure 6.12: Dimensionless torque and DR as a function of $\mathrm{Re}_{i}$. The reference case is without cavitators and with $\alpha=0$. The other cases are measured with 3 cavitators. The outer cylinder is stationary. (a) The dimensionless torque as a function of the inner Reynolds number $\operatorname{Re}_{i}$. (b) The net drag reduction as a function of the inner Reynolds number $\operatorname{Re}_{i}$. The net DR as compared to the reference case is negative, i.e. instead of drag reduction we observe a drag increase. A typical error bar is shown in both graphs.

As discussed in the introduction, one has to find the optimum between the DR caused by the cavities and the drag increase caused by the cavitators due to their pressure drag. In figures 6.86 .10 the presented DR percentages are relative to the case without air, but with cavitators. The presented DR values can be seen as a 'gross drag reduction'. However, it is known that in TC flow (as for any other flow) even small roughness heights increase the drag tremendously 125,220 . The cavitators have a height of $2 \mathrm{~mm}$, corresponding to $2.5 \%$ of the gap width and to $\mathcal{O}\left(10^{2}\right)$ wall units, so we are in the fully rough regime. In this paragraph, we study the effect of these cavitators on the drag, by comparing our results with a reference case without cavitators. We define a 'net DR' as:

$$
\mathrm{DR}_{\text {net }}=1-G(\alpha) / G_{r e f}
$$

in which $G_{r e f}$ is a reference case without air and without cavitators, i.e. with smooth cylinders. The results are shown in fig. 6.12. Clearly, the lowest dimensionless torque is obtained with the reference case. So, when applying air cavities, the net drag is increased, as is also clear from fig. 6.12 b. We observe here that the drag increase caused by the cavitators is larger than the drag reduction caused by the air layer. We, however, note that measuring at larger void fractions and Reynolds 
numbers might cause $D R_{n e t}$ to be positive. Knowing the difference between net and gross drag reduction is crucial when applying air cavities. Full-scale ship experiments are extremely costly, and a reference test without cavitators might not be performed at all. Here, we show that the negative effects of the cavitators can be larger than the beneficial effects of the air cavity.
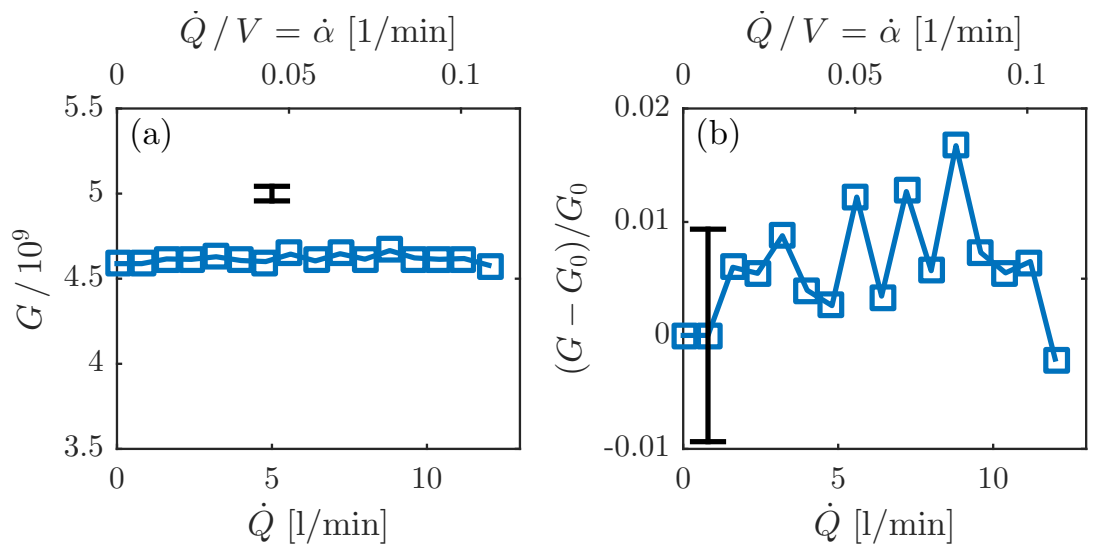

Figure 6.13: (a) The dimensionless torque $G$ as a function of gas flow rate $\dot{Q}$. We observe that $G$ does not depend on $\dot{Q}$. Here, the number of cavitators is 3 , and we measure at $\operatorname{Re}_{i}=1 \times 10^{6}$ with a void fraction of $\alpha=2 \%$. The OC is kept stationary. On the top x-axis, we non-dimensionalized $\dot{Q}$ with the volume of the system $V$, which equals $\dot{\alpha}$, i.e. the void fraction injected per minute. Injecting more air than shown here is not possible as it leads to an increase in void fraction as water is pushed out of the system, and thus to an unfair comparison. In plot (b), we show the same data but made dimensionless: $\left(G-G_{0}\right) / G_{0}$, in which $G_{0}=G(Q=0)$. In this way, the relative change of $G$ is shown. A typical error bar is shown in both graphs.

In water tunnel measurements the parameter governing the amount of air in the flow is the air injection rate $\dot{Q}$, which partially governs the cavity stability 134$]. \dot{Q}$ should be sufficiently high to maintain the cavity, whereas a further increase in $\dot{Q}$ does not further increase the cavity length, but causes more air to be discharged in the closure region. In a closed TC system, air escaping from a certain cavitator can be re-entrained by other cavitators downstream. Similarly, in flat plate experiments it was shown that air which is discharged from a cavity can develop a new cavity if an additional cavitator is placed downstream [134]. In open systems, such as ships or flat plates, the working fluid (with $\alpha=0 \%$ ) is continuously refreshed such that $\dot{Q}$ is important, while the TC flow system is closed, meaning that $\alpha$ is the relevant parameter. In all measurements presented above, we did not inject air locally, but instead chose to fill the cylinder only partially (see fig. 6.1). Air is then entrained in the water by turbulent mixing. It is not known to which extent active local gas injection influences the global torque and the air cavity length, given a certain $\alpha$. The fact that pipe flow measurements showed that air can be reentrained at a cavitator placed 
further downstream indicates that rather than the air injection rate, the amount of available air is crucial [134]. However, in flat plates it is not possible to disentangle air injection and local void fraction. Here we study this by injection of a certain gas flow rate $\dot{Q}$, locally, directly at the cavitator, as indicated in fig. 6.2 , while keeping the void fraction constant at $\alpha=2 \%$ and the Reynolds number constant at $\operatorname{Re}_{i}=1 \times 10^{6}$. We simultaneously measure the torque. We note that we inject significant amounts of air as compared to the amount of air which already is in the system (2.2 liter for $\alpha=2 \%$ ), namely up to 5 times as much air per minute than the amount of air already present.

Surprisingly, we observe that active air injection does not influence the torque in the studied range of air injection rates, as shown in fig. 6.13. Apparently, the turbulent mixing in the flow is so strong that a steady state is reached almost immediately. Therefore, any excess of air is transported towards the top of the setup immediately, where it can leave the system. This is somewhat similar to what was found in [134]. In here, it was observed that increasing the gas flow in a flat plate setup does not increase the length of the cavity, and only leads to an increase in air discharge in the closure region.

\subsection{Discussion and conclusions}

A relevant question one could ask is: how can these Taylor-Couette results be compared with channel flow measurements [2, 214, 217]? Several parameters which are common in the channel flow- and naval architecture communities are not used in TC flow and vice versa, see table 6.1 for a comparison of these parameters. As discussed above, as the air is not continuously swept away, like in an open system, it is $\alpha$, rather than $\dot{Q}$, that is the governing parameter in a closed flow system. The mean-field forcing of the flow is a second source of ambiguity. In TC flow, the mean-field forcing is not limited to the gravitational forces, as the centrifugal forces play a large role. The centrifugal forces $a_{\text {centr }}=\omega_{i}^{2} r_{i}$ increase with Reynolds number until eventually, in the limit of $\operatorname{Re}_{i} \rightarrow \infty, a_{\text {centr }} \gg g$. As the centrifugal forces are directed in the radial direction, the water depth is to be taken in radial direction as well and thus equals the gap width $d$. Furthermore, in TC flow a second Froude number can be defined. The commonly used depth-based Froude number is defined as $\mathrm{Fr}=\frac{u_{\infty}}{\sqrt{g D}}$, in which $u_{\infty}$ is a free stream velocity and $D$ is the water depth. Using the centrifugal acceleration we now define a "centrifugal Froude number" as $\operatorname{Fr}_{c e n t r}=\frac{u_{i}}{\sqrt{a_{\text {centrd }}}}=\sqrt{r_{i} / d}=\sqrt{\eta /(1-\eta)}$, which, surprisingly, does not depend on the driving of the setup but only on the geometrical parameter $\eta$. In the currently used setup, the centrifugal Froude number equals $\operatorname{Fr}_{\text {centr }}=1.59$.

Earlier studies showed that the length of the cavity equals half of the gravity wavelength, which is described by the dispersion relation $u_{\infty}=\sqrt{\frac{g \lambda}{2 \pi} \tanh \frac{2 \pi D}{\lambda}}$, in which $\lambda$ is the wavelength of the surface gravity waves 130,132 . Here, as $\operatorname{Fr}_{\text {centr }}>1$, the flow is supercritical, and the gravity wavelength becomes infinite [134]. Thus, if enough air would be available and for $a_{c e n t r} \gg g$, our streamwise cavity length would 


\begin{tabular}{lll} 
Quantity & Channel flow & TC flow \\
\hline Amount of gas & $\dot{Q}[1 / \mathrm{s}]$ & $\alpha[\%]$ \\
Gravity & $\operatorname{Fr}$ & For high $\operatorname{Re}_{i}: \operatorname{Fr}_{c e n t r}$. For low $\operatorname{Re}_{i}:$ Fr \\
Water depth & $\mathrm{D}$ & For high $\operatorname{Re}_{i}: r_{o}-r_{i}$. For low $\operatorname{Re}_{i}: L$ \\
Cavitation number & $\sigma$ & $\sigma_{T C}$ \\
Driving & $\operatorname{Re}$ & $\operatorname{Re}_{i}$ and $\operatorname{Re}_{o}$
\end{tabular}

Table 6.1: Comparison between air cavity parameters for channel flow and TC flow.

become unbounded and the entire cylinder is expected to be covered in air. We note that in our study, both the buoyancy forces and centrifugal forces play a role, and therefore the flow is not yet supercritical, and consequently, the cylinder is not yet fully covered by cavities. In channel flow, the cavitation number $\sigma$ is one of the basic parameters, and it is straightforward to measure. It is defined as $\sigma=\left(p-p_{c}\right) /\left(\frac{1}{2} \rho u_{\infty}^{2}\right)$, in which $p$ is the free stream pressure and $p_{c}$ is the pressure in the cavity. In TC flow, due to hydrostatic pressure, we define a height dependent cavity number as $\sigma_{T C}=\left(p(z)-p_{c}\right) /\left(\frac{1}{2} \rho u_{i}^{2}\right)$.

A final difference between TC flow and channel flow is the way the systems are driven. Channel flow is pressure-driven, and consequently the momentum is transported from bulk to BLs. In TC flow, momentum is transported from the inner cylinder BL to the outer cylinder BL. The regimes of counter- and co-rotation, caused by rotation of the outer cylinder are exclusive to the TC geometry.

Then, knowing these differences, what can be learned from these experiments, and how can these interpreted and applied by the naval industry? Our conclusion is that although one-to-one comparisons are difficult, the underlying physics remains the same. Therefore, our findings are of interest to anyone working on this topic.

To conclude, in this chapter we convincingly showed that air cavities can be (re)entrained in a Taylor-Couette flow setup. We show that air cavities result in gross DR percentages which are larger than the DR percentages for conventional bubble drag reduction. However, for all cases we see a net drag increase, caused by pressure drag at the cavitators. Therefore, when applying air cavities it is crucial to focus on the balance between drag reduction by the cavities and drag increase by the cavitators, closure region and any skegs. In addition, for maritime applications one should also take into consideration the energy costs to continuously inject air to judge whether or not a net gain can be achieved.

We observed that the streamwise cavity length is significantly influenced by buoyancy effects. Therefore, we expect that air cavities on any non-flat bottomed hull behave similarly, and applying them is difficult. The global coverage is correlated to the Reynolds number and void fraction. To conclude, we showed that local air injection is not necessary, as long as sufficient amounts of air are available. This confirms that air which is discharged can be captured by any cavitator placed downstream on the hull.

In this exploratory study we restricted ourselves to one cavitator shape. Future 
work includes a study on the shape and size of the cavitators, preferably measuring at higher Reynolds numbers or at larger void fractions. The flow can be further quantified by local velocity measurements, which, although these are clearly difficult in multiphase flows, should be possible as the air is not dispersed homogeneously throughout the flow domain. 


\section{Chapter 7}

\section{The influence of wall roughness on bubble drag reduction in Taylor-Couette turbulence ${ }^{1}$}

We experimentally study the influence of wall roughness on bubble drag reduction in turbulent Taylor-Couette flow, i.e. the flow between two concentric, independently rotating cylinders. We measure the drag in the system for the cases with and without air, and add roughness by installing transverse ribs on either one or both of the cylinders. For the smooth wall case (no ribs) and the case of ribs on the inner cylinder only, we observe strong drag reduction up to $D R=33 \%$ and $D R=23 \%$, respectively, for a void fraction of $\alpha=6 \%$. However, with ribs mounted on both cylinders or on the outer cylinder only, the drag reduction is weak, less than $D R=11 \%$, and thus quite close to the trivial effect of reduced effective density. Flow visualizations show that stable turbulent Taylor vortices - large scale vortical structures — are induced in these two cases, i.e. the cases with ribs on the outer cylinder. These strong secondary flows move the bubbles away from the boundary layer, making the bubbles less effective than what had previously been observed for the smoothwall case. Measurements with counter-rotating smooth cylinders, a regime in which pronounced Taylor rolls are also induced, confirm that it is really the Taylor vortices that weaken the bubble drag reduction mechanism. Our findings show that, although bubble drag reduction can indeed be effective for smooth walls, its effect can be spoiled by e.g. biofouling and omnipresent wall roughness, as the roughness can induce strong secondary flows.

\footnotetext{
${ }^{1}$ Ruben A. Verschoof, Dennis Bakhuis, Pim A. Bullee, Sander G. Huisman, Chao Sun, and Detlef Lohse, The influence of wall roughness on bubble drag reduction in Taylor-Couette turbulence, under review.

Experiments by Verschoof, Bakhuis and Bullee. Verschoof analysed the data and wrote the paper. Huisman, Sun and Lohse supervised the project. All authors discussed the results and proofread the paper.
} 


\subsection{Introduction}

In the maritime industry, air lubrication is seen as one of the most promising techniques to reduce the overall fuel consumption [2, 101, 138]. Air lubrication has been studied for several decades, and it is found that a few percent of air can significantly decrease the overall friction, e.g. with $4 \%$ bubbles, drag reductions up to $40 \%$ were shown 9,126]. Notwithstanding its clear industrial potential, it remains difficult to translate highly controlled laboratory results to drag reduction in large-scale vessels. So far, the vast majority of studies on bubble drag reduction (DR) have been performed in test facilities with purified water and smooth walls, see e.g. the review articles Ceccio 217] and Murai 214]. However, many surfaces in industry are rough to some extent, and also initially smooth surfaces can become rough by means of corrosion, cavitation, mineral scaling, and (bio)fouling [85,104]. Furthermore, the dynamics of bubbles are strongly affected by any dissolved ions in oceanic water and surfactants 194,203. As the conditions in controlled experiments and real applications are so much different, one can expect that bubble DR experiments will lead to very different results in practice. Only a limited number of studies focussed on 'non-ideal' DR, either through wall roughness [196, 209, 210], surfactants or seawater 102, 105, 106, 126, 209, and their results are somewhat inconsistent. Some studies found that wall roughness completely eliminates any drag reduction 210, whereas others show that roughness does not affect, or even enhance, drag reduction [196,209]. Therefore, there is a clear need to better understand the influence of wall roughness on bubble drag reduction.

In this work, we study the effect of wall roughness on bubble drag reduction. To do so, we employ the Taylor-Couette (TC) system, i.e. the flow between two concentric, independently rotating cylinders $[50,51]$. TC flow is one of the canonical flow systems in which fluid mechanics concepts and theories are tested. Among the advantages of using a TC setup are the ease with which the global void fraction $\alpha$ is controlled, the absence of any streamwise spatial transients, and as it is a closed system, an exact balance that connects the global torque measurements with the local energy dissipation rate. The driving and response of the system are characterized by the Taylor number Ta and the Nusselt number $\mathrm{Nu}_{\omega}$, respectively [51]. The Nusselt number is defined as the ratio of the convective momentum transport to the diffusive flux, and using it underlines the close analogy between Taylor-Couette flow and Rayleigh-Bénard convection 189 . In the currently studied parameter regime, an effective scaling of $\mathrm{Nu}_{\omega} \propto \mathrm{Ta}^{0.4}$ is observed 4]. The TC setup has been used frequently to study (bubble) drag reduction in turbulent flows $9,11,126,127,129$, even numerically 93,115 . In these studies, it was shown that a small air fraction can considerably reduce the drag. E.g. with a void fraction of $\alpha=4 \%$, a drag reduction of $40 \%$ was observed $[9,126$, which is significantly larger than the trivial effects of affected effective density and viscosity. These studies highlighted the importance of bubble deformability for large drag reduction, and thus a sufficiently large Weber number We $=\rho D_{b} u^{2} / \sigma$, in which $\rho$ is the fluid density, $D_{b}$ the bubble diameter, $u$ a characteristic velocity and $\sigma$ the interfacial surface tension. It was shown that large Weber number bubbles, i.e. large 
and deformable bubbles, are crucial to efficiently reduce the drag.

Wall roughness, on the other hand, obviously increases the friction. Its effects have been studied extensively for single-phase turbulence flows, mostly in pipe or channel flow configurations given their industrial relevance, see e.g. refs. [45, 151 and references therein. For TC flow, adding sufficiently large, rough ribs results in $\mathrm{Nu}_{\omega} \propto \mathrm{Ta}^{1 / 2}$ scaling, rather than the aforementioned $\mathrm{Nu}_{\omega} \propto \mathrm{Ta}^{0.4}$ smooth wall scaling $96,125,220$. The $\mathrm{Nu}_{\omega} \propto \mathrm{Ta}^{1 / 2}$ scaling, mathematically equivalent to a constant friction coefficient in the fully rough regime, is the mathematical upper bound to the transport of momentum. In this regime, the roughness decreases the near wall velocity gradient, whereas the streamwise velocity fluctuations are increased. The bubble dynamics are largely governed by the motion of the surrounding fluid, but to which extent any drag reduction is affected by the changed fluid motion is unknown.

A number of studies focussed on wall modifications to stimulate air to attach to the inner cylinder wall of Taylor-Couette flow, either by a hydrophobic coating [128 or by using cavitors to try to create an air layer [103]. van den Berg et al. 210] studied the effects of roughness on bubble drag reduction, and found that ribs attached to both cylinders prevent bubbles from reducing the overall friction. Therefore, it was suggested that bubbly drag reduction is a boundary layer effect. However, the exact reason why the drag reduction was lost remained elusive. Therefore, here we aim to repeat and extend those experiments in a more accurate and controlled setup and to visualize the flow, to better understand the physics of the aforementioned conclusions.

\subsection{Experimental method}

The experiments are performed in the Twente Turbulent Taylor-Couette facility $\left(\mathrm{T}^{3} \mathrm{C}\right) 3$, in which the flow is fully turbulent. TC flow is driven by the angular velocity of the inner and outer cylinder, denoted by $\omega_{i}$ and $\omega_{o}$, respectively. The setup has a height of $L=927 \mathrm{~mm}$, an inner radius $r_{i}=200 \mathrm{~mm}$, an outer radius $r_{o}=279.4 \mathrm{~mm}$, giving a gap width $d=r_{o}-r_{i}=79.4 \mathrm{~mm}$. The geometry can therefore be described by two geometric parameters; the radius ratio $\eta=r_{i} / r_{o}=0.716$ and the aspect ratio $\Gamma=L / d=11.7$, see also figure 7.1a. The inner cylinder and outer cylinder rotate up to $f_{i}=\omega_{i} /(2 \pi)=10 \mathrm{~Hz}$ and $f_{o}=-4 \mathrm{~Hz}$, respectively. These result in two Reynolds numbers: $\operatorname{Re}_{i, o}=\omega_{i, o} r_{i, o}\left(r_{o}-r_{i}\right) / \nu$, respectively, in which $\nu$ is the viscosity of the working fluid, and a rotation ratio $a=-\omega_{o} / \omega_{i}$. We here express the driving using the Taylor number Ta $=\left[(1+\eta)^{4} /\left(64 \eta^{2}\right)\right] d^{2}\left(r_{i}+r_{o}\right)^{2}\left(\omega_{i}-\omega_{o}\right)^{2} \nu^{-2} \propto\left(\operatorname{Re}_{i}-\eta \operatorname{Re}_{o}\right)^{2}$, which thus incorporates the rotation of both cylinders in one dimensionless number. In the current study, we measure at Taylor numbers of $\mathcal{O}\left(10^{12}\right)$, or, equivalently, Reynolds numbers up to $\mathcal{O}\left(10^{6}\right)$. The primary response parameter is the torque $\tau$ necessary to maintain the inner cylinder at a constant angular velocity. The torque is measured with a co-axial torque transducer (Honeywell Hollow Reaction Torque Sensor 2404-1K, maximum capacity of $115 \mathrm{Nm}$ ), which is located inside the inner cylinder to avoid measurement errors due to seal and bearing friction, see figure $7.1 \mathrm{p}$. The 
(a)

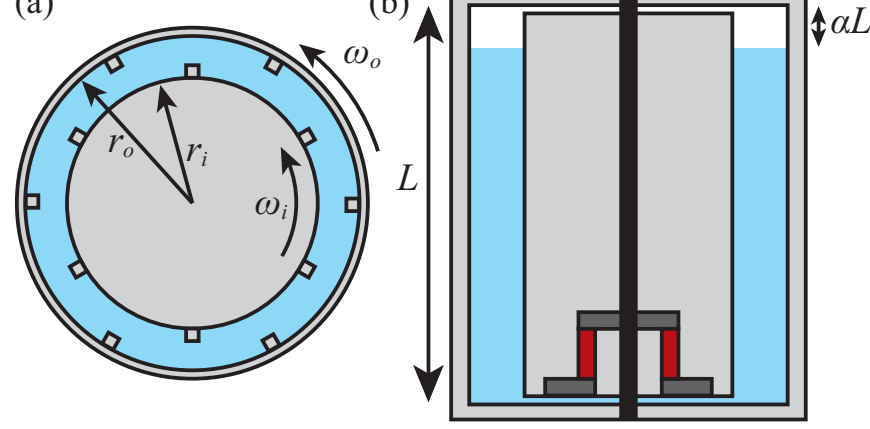

(c)

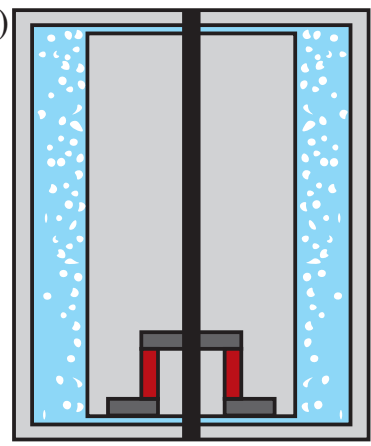

Figure 7.1: Experimental setup. (a) Top view schematic of the $\mathrm{T}^{3} \mathrm{C}$ facility. We attached 6 vertical transverse ribs (not to scale) equally distributed around the perimeter of the inner cylinder, the outer cylinder, or both cylinders. We also measure a smooth-wall case without any ribs. (b) Vertical cross-section of the setup at rest, showing the position of the torque sensor. To control the void fraction, we fill the cylinder only partially with water, so that the void fraction $\alpha$ is controlled by measuring the relative height of the water level. (c) Vertical cross-section of the setup during a measurement. The free surface disappears, and all air is entrained by the turbulent flow (bubbles not to scale).

torque is made dimensionless with the torque for laminar non-vertical flow, resulting in the Nusselt number: $\mathrm{Nu}_{\omega}=\tau / \tau_{\text {lam }}$, with $\tau_{\text {lam }}=4 \pi L \rho \nu r_{i}^{2} r_{o}^{2}\left(\omega_{i}-\omega_{o}\right) /\left(r_{o}^{2}-r_{i}^{2}\right)$. The flow is cooled through both endplates to counteract viscous heating, keeping the water temperature constant within $T=21 \pm 0.5^{\circ} \mathrm{C}$. Although the effective viscosity and density are altered by the presence of bubbles, we chose to consequently use the pure water material properties, as we are interested in the net changes in drag.

The cylinders are made rough by attaching 6 transverse ribs over the entire height of the cylinders, as shown in figure 7.1 a. The rib dimensions are $6 \mathrm{~mm}$ by $6 \mathrm{~mm}$, corresponding to $7.5 \%$ of the gap width, and to $\mathcal{O}\left(10^{3}\right)$ in wall units, depending on the Taylor number and roughness case. We study the torque and resulting drag reduction for 4 cases: both cylinders smooth (SS), both cylinders rough (RR), and roughness on either only the inner cylinder (RS) or only on the outer cylinder (SR). We here chose to apply rib roughness, which, for the RR case, causes the flow to be in the "fully rough" state, or the "asymptotic ultimate turbulence" regime [220] in the studied parameter regime. In this regime, the behaviour in the boundary layers becomes independent of the viscosity. Consequently, a $\mathrm{Nu}_{\omega} \propto \mathrm{Ta}^{1 / 2}$ is observed rather than the effective $\mathrm{Nu}_{\omega} \propto \mathrm{Ta}^{0.4}$ scaling found for the smooth wall case in the currently studied parameter regime $4,185,220$. For the cases of ribs on a single cylinder, the exponent $\gamma$ of the $\mathrm{Nu}_{\omega} \propto \mathrm{Ta}^{\gamma}$-scaling is between these two bounds.

The gap is either partially or completely filled with water, so that the void fraction is precisely set between $0 \% \leq \alpha \leq 6 \%$, see figure $7.1 \mathrm{~b}$. We determine the void fraction with both cylinders at rest. During a flow measurement, the air is distributed over the height of the cylinder because of turbulent mixing, see figure 7.1k. We note that 

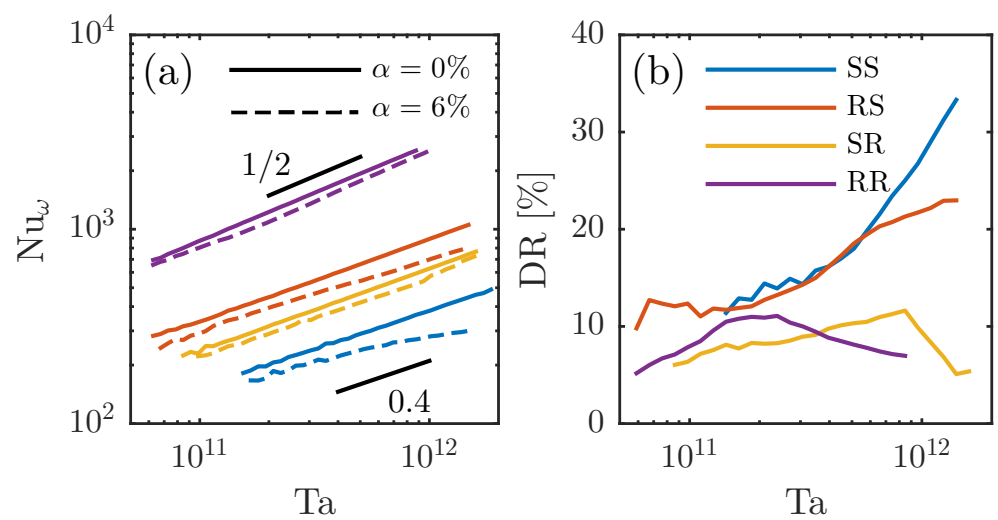

Figure 7.2: (a) Dimensionless angular velocity flux $\mathrm{Nu}_{\omega}$ as a function of Ta for $\alpha=0 \%$ and $\alpha=6 \%$. To increase the readability, we do not show $\mathrm{Nu}_{\omega}$ for $\alpha=\{2 \%, 4 \%\}$, which are used to calculate the DR shown in figure 7.3 . The two short black lines indicate the $\mathrm{Nu}_{\omega} \propto \mathrm{Ta}^{\gamma}$ scaling relations for the pure liquid cases. The exponents are $\gamma=0.4$ and $\gamma=1 / 2$ for the SS and RR cases, respectively. (b) Resulting drag reduction as a function of $T a$. The outer cylinder is stationary. The DR is calculated with equation 7.1.

a perfect homogeneous axial distribution is not feasable, even with continuous bubble injection through the bottom end cap [9], but it becomes more homogeneous with increasing Taylor number.

\section{$7.3 \quad$ Results}

We measure the torque and present our findings in figure 7.2 and figure 7.3 . The drag reduction is calculated as

$$
\mathrm{DR}=1-\frac{\mathrm{Nu}_{\omega}(\alpha)}{\mathrm{Nu}_{\omega}(\alpha=0)},
$$

in which we compare the $\mathrm{Nu}_{\omega}$ values for the same roughness case. In figure 7.2 , we show the Nusselt number and resulting drag reduction for all roughness cases. As was shown before, $\mathrm{Nu}_{\omega}$ depends tremendously on the applied roughness 220]. In the current study, however, we are more interested in the relative bubbly drag reduction as compared to the smooth wall case, rather than the absolute friction increase by roughness. From figure 7.2 two different regimes can be distinguished: we observe strong drag reduction for the SS and RS cases - up to DR $=33 \%$ with a void fraction of $\alpha=6 \%$, whereas for the SR and RR cases, the DR is only weak - with the same void fraction never exceeding $\mathrm{DR}=12 \%$.

To further study the DR per roughness case, we show the drag reduction for void fractions of $2 \%, 4 \%$ and $6 \%$ in figure 7.3 . The DR increased monotonically with increasing void fraction for all cases. In the weak drag reduction cases (SR and RR), 

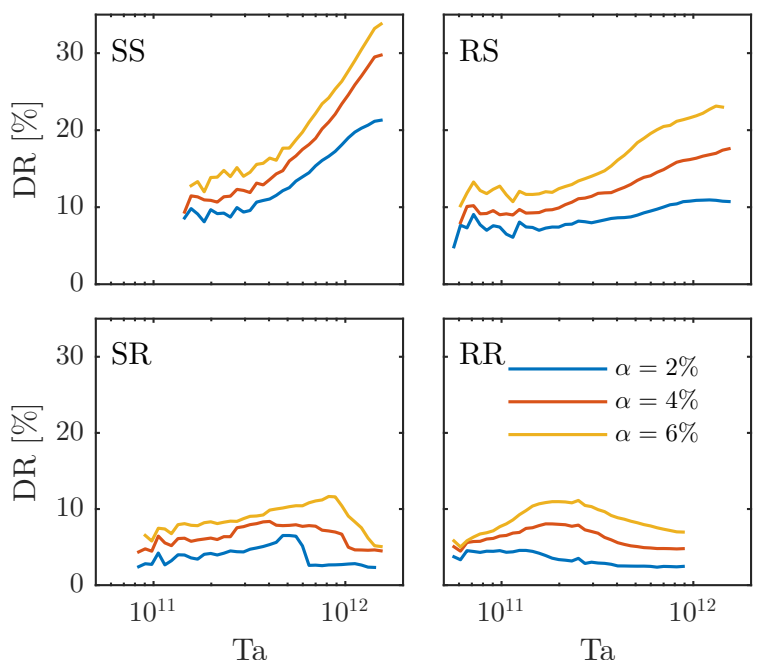

Figure 7.3: Drag reduction percentages as a function of Ta for all roughness cases. In the RR case, the highest achievable Taylor number is slightly smaller due to experimental limitations. The outer cylinder is stationary. The DR is calculated with equation 7.1

the DR is quite close to the trivial effect of reduced global density, which equals $\rho_{\text {eff }}=\rho(1-\alpha)+\alpha \rho_{\text {air }} \approx \rho(1-\alpha)$, in which $\rho_{\text {air }}$ is the air density. For the RS and SS cases however, the drag reduction is significantly larger than the reduced density effect. Interestingly, given the strong DR in the RS case, it is clear that wall roughness does not necessarily prevents strong bubble drag reduction. For both the RS and SS cases, the drag reduction increases with Taylor number [9], contrasting the SR and $\mathrm{RR}$ cases, in which the drag reduction does not have a clear monotonic Taylor number dependence.

To better understand the flow dynamics, we visualize the flow for a void fraction of $\alpha=6 \%$. As shown in figure 7.4. for all four cases the flow structures are significantly different. In the SS and RS cases, clear streaks and patterns are visualized by the bubbles, but stable turbulent Taylor vortices are not observed. For both cases with ribs on the outer cylinder, i.e. the SR and RR cases, we do however observe stable turbulent Taylor vortices.

The existence and the dynamics of Taylor vortices have been studied extensively for the single-phase smooth-wall case $6,6,7,51,53,107]$. In the explored Taylor number regime, measurements showed that for sufficiently strong turbulence $\left(\operatorname{Re}_{i}>10^{5}\right)$, stable Taylor rolls do not exist in the pure inner cylinder rotation regime, and are only present in the counter-rotating regime 5,51. Roughness elements promote the ejection of turbulent plumes, leading to localised radial flows towards the outer cylinder [108,159. As the TC system is closed, consequently a radial flow towards the inner cylinder must be present. These flows can organise themselves as stable 

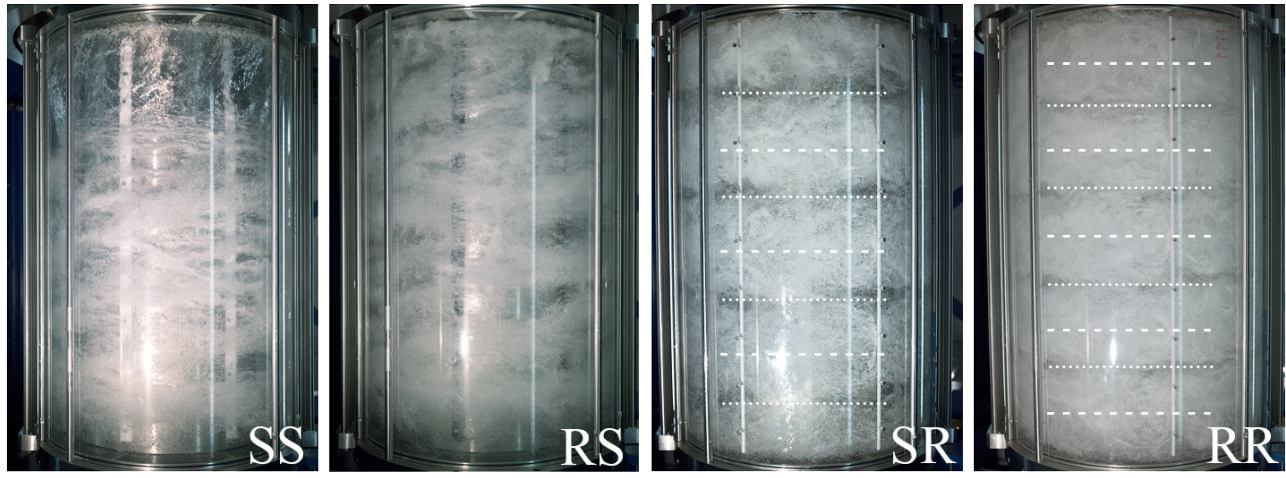

Figure 7.4: Instantaneous photographs of the flow for all four roughness cases: SS, RS, SR and RR. Clear differences in the flow patterns are visible. In the SS and RS cases, we see turbulent streaks, but no stable structures. Clear stable Taylor rolls are visible for the SR and RR cases. We indicate the position of the rolls by the dashed line, and indicated the roll pairs by the dotted line. The Taylor number is $\mathrm{Ta}=1.5 \times 10^{12}$, except for the $\mathrm{RR}$ case $\left(\mathrm{Ta}=8.4 \times 10^{11}\right)$, while the outer cylinder is kept stationary. The void fraction in all cases is $\alpha=6 \%$. Note that in all cases the bubbles are not homogeneously distributed over the height, this is most visible in the SS case.

Taylor rolls. Thus, as the roughness promotes the ejection of turbulent plumes, the existence of Taylor vortices is stimulated.

The roll dynamics observed with wall roughness are different than what has been observed hitherto in the same setup. Earlier studies found 6 or 8 rolls for the smoothwall case with counter-rotating cylinders [52,107]. Here, for pure inner cylinder rotation, we see 10 rolls for the RR case, whereas for the SR case we observe 8 rolls. The number of rolls is related to the aspect ratio $\Gamma$, which depends on the gap width. The roughness elements decrease the 'effective gap width', and thus increase the apparent aspect ratio $\Gamma_{\text {eff }}$. Therefore, the system allows for an increased number of rolls 107

We argue that the existence of the Taylor vortices is the underlying mechanism through which the effectiveness of bubble drag reduction is reduced in the SR and $\mathrm{RR}$ cases. To effectively decrease the drag, it is crucial that large bubbles are present in or close to the boundary layer $9,93,126$. The flow visualizations show that the bubbles are dragged away from the inner cylinder wall by low vorticity regions, here in the form of turbulent Taylor vortices. Therefore, as the bubbles do not accumulate close to the inner cylinder, the drag reduction almost vanishes, and becomes close to the trivial effect of the reduced global effective density, as was shown in our torque measurements.

Up to here, we showed that in the SR and RR cases, the ribs induce turbulent Taylor vortices, and we argued that Taylor rolls eliminate DR. One could presume that the rolls, instead of being the underlying physical explanation, merely coincide with the weak DR. To further prove the effect of turbulent Taylor vortices on bub- 

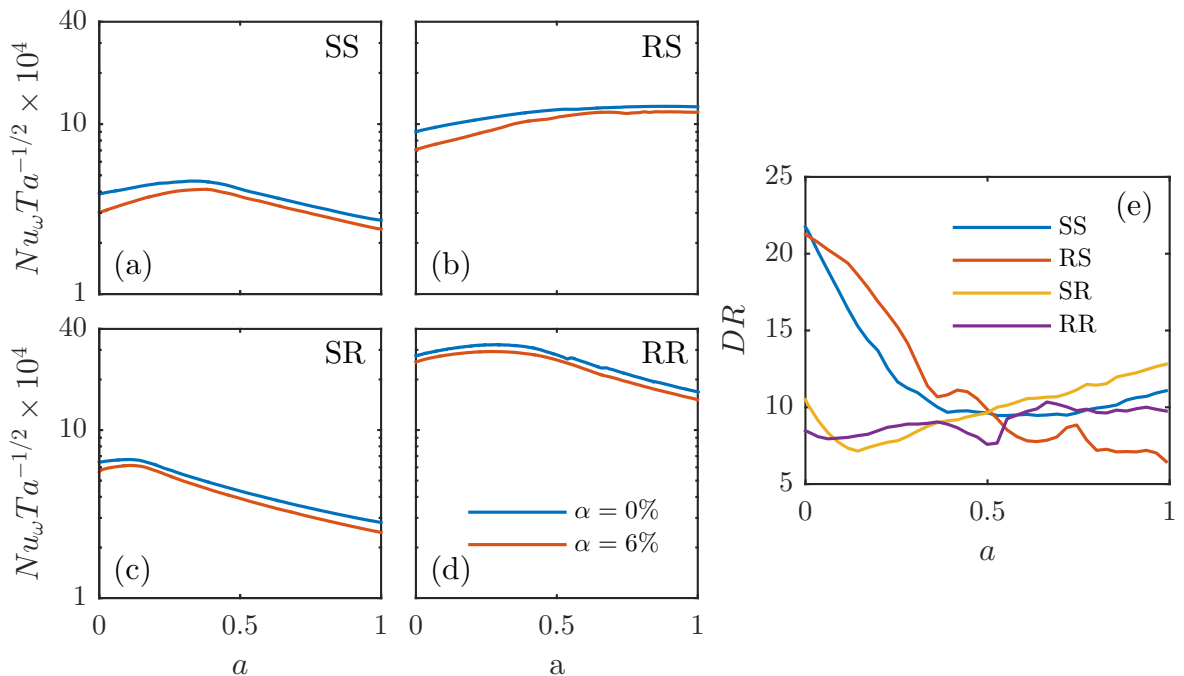

Figure 7.5: (a-d) Dimensionless torque as a function of rotation ratio $a$ for the roughness cases SS, SR, RS, and RR with and without air. The Taylor number is kept constant at $\mathrm{Ta}=7.3 \times 10^{11}$. We compensate $\mathrm{Nu}_{\omega}$ by $\mathrm{Ta}^{0.5}$ to remove viscosity changes due to temperature fluctuations, similar as in ref. [52]. (e) Resulting drag reduction as a function of rotation ratio $a$, calculated here as $\mathrm{DR}=1-\frac{\mathrm{Nu}_{\omega} \mathrm{Ta}^{-0.4}(\alpha=6 \%)}{\mathrm{Nu}_{\omega} \mathrm{Ta}^{-0.4}(\alpha=0 \%)}$.

ble DR, we study the DR behavior in the counter-rotation regime. For the SS case, pronounced stable turbulent Taylor vortices exist in the counter-rotating regime between approximately $0.1 \leq a \leq 0.5[52,107,176]$. By measuring the DR as a function of rotation ratio $a=-\omega_{o} / \omega_{i}$ while keeping the Taylor number constant, we can directly show the influence of Taylor rolls on the effectiveness of air lubrication. For all roughness cases, we show the torque in figure $7.5(\mathrm{a}-\mathrm{d})$ and the resulting DR in figure 7.5 (e). As already shown before, we observe strong DR for the SS and SR cases at $a=0$. Then, for increasing $a$, we see that the DR decreases. The observation is very similar to the above discussed weak DR in the SR and RR cases, namely in the counter-rotating regime the bubbles are trapped in the Taylor rolls, dragged away from the boundary layer and unable to effectively decrease the drag. For the SR case, the strength of the turbulent Taylor vortices decreases with increasing outer cylinder. Consequently, an increase in DR is observed. For the RR case, the DR remains weak for all cases, as the turbulent Taylor vortices exist for the entire scanned parameter space.

\subsection{Conclusions}

To conclude, we studied the influence of wall roughness on bubble drag reduction in a highly turbulent flow. We showed that in the SR and RR cases wall roughness 
promotes stable turbulent Taylor rolls, which induce strong secondary flows, suppressing the drag reduction. Bubbles are captured in low vorticity regions, and therefore dragged away from the inner cylinder boundary layer. As a result, the drag reduction is mostly lost, and the effective drag reduction is close to the trivial effect of reduced global density. These findings help us to understand earlier studies on air lubrication and wall roughness, which had conflicting results whether roughness influences bubble drag reduction. We here distinguish two different regimes: (i) a regime with strong drag reduction if the roughness does not introduce strong secondary flows. And, (ii) a regime with weak drag reduction if strong secondary flows are induced by the roughness.

Future work includes studies on wall roughness combined with bubbles in other types of setups, e.g. flat plates, or pipe flow. In these setups, roughness increases the velocity fluctuations but not necessarily induces stable large-scale secondary flows, and thus the bubble DR behaviour might be significantly different than in the current study. Moreover, as we here limited ourselves to the influence of rib roughness, more work is needed to understand the influence of more realistic types of roughness. 


\section{Conclusions}

In this thesis we studied various forms of non-ideal turbulent flows, i.e. multiphase flows, wall roughness and transient effects. As test setup we used a high Reynolds number Taylor-Couette setup, in which we performed both local and global flow measurements. The findings of each chapter are discussed below.

In chapter 1 we studied the decay of high Reynolds number Taylor-Couette flow. We showed that decay in this inhomogeneous turbulent flow does not follow a strict power law as for decaying homogeneous isotropic flows, as the decay is faster due to the viscous drag applied by the walls. The radial profile of the azimuthal velocity is found to be self-similar over time. Furthermore, the flow velocity does not develop any height dependence, in contrast to the well-known Taylor vortex state.

In chapter 2 we sinusoidally drove the inner cylinder of the Taylor-Couette system, thus periodically forcing the flow. Using particle image velocimetry, we measured the velocity over a wide range of modulation periods $T$, non-dimensialized as the Womersley number by $\alpha=d \sqrt{2 \pi /(T \nu)}$. To understand how the flow responds to a given modulation, we calculate the phase delay and amplitude response of the azimuthal velocity. In agreement with earlier theoretical and numerical work, we find that for large modulation periods the system follows the given modulation of the driving, i.e. the system behaves quasi-stationary. For smaller modulation periods, the flow cannot follow the modulation, and the flow velocity responds with a phase delay and a smaller amplitude response to the given modulation. Surprisingly, the local response in the bulk of the flow is independent of the distance to the modulated boundary. Apparently, the turbulent mixing is strong enough to prevent the flow from having radius-dependent responses to the given modulation.

In chapter 3 we studied the effects of wall roughness on turbulent flows. By combining extensive experiments and numerical simulations, here, taking as example the Taylor-Couette system, we show how wall roughness greatly enhances the overall transport properties and the corresponding scaling exponents. If only one of the walls is rough, we reveal that the bulk velocity is slaved to the rough side, due to the much stronger coupling to that wall by the detaching flow structures. If both walls are rough, the viscosity dependence is eliminated in the boundary layers and we thus achieve asymptotic ultimate turbulence, i.e. the upper limit of transport, whose existence had been predicted by Robert Kraichnan in 1962 (Phys. Fluids 5, 1374 (1962)) and in which the scalings laws can be extrapolated to arbitrarily large 
Reynolds numbers.

In chapter 4 we built upon these results by varying the rib height, and focussed on the local flow properties and the counter-rotating regime. We showed the impact of riblet height and number of ribs on the global torque $\tau$, and find an effective scaling of $\tau \propto n_{r} h^{1.71}$, in which $n_{r}$ is the number of ribs and $h$ the rib height. Even for huge rib heights, the behaviour in the counter-rotating regime is largely unaffected: in this regime Taylor vortices increase the torque, and we observe a rotation ratio $a_{\text {opt }}$ for which the torque reached a maximum value.

In chapter 5 we convincingly showed that bubble drag reduction dramatically depends on the bubble size. We added minute concentrations of the surfactant Triton X-100 into otherwise completely unchanged strongly turbulent Taylor-Couette flow containing bubbles, we dramatically reduce the drag reduction from more than $40 \%$ to about $4 \%$, corresponding to the trivial effect of the bubbles on the density and viscosity of the liquid. The reason for this striking behavior is that the addition of surfactants prevents bubble coalescence, leading to much smaller bubbles. Our result demonstrates that bubble deformability is crucial for bubble drag reduction in turbulent flow and opens the door for an optimization of the process.

In chapter 6 we explored the possibilities of studying air cavities in Taylor-Couette turbulence. An air cavity, i.e. an air layer developed downstream an rised edge - the cavitator - is seen by the maritime industry as a promising method to reduce the overall skin friction acting on ships. We showed that creating air cavities in TC flow is feasible, and that they result in significant gross drag reductions. However, the added drag due to the cavitators is larger than the drag reduction by the cavities, so that in fact a net drag increase was observed. We demonstrated that local air injection is not important, air should be present in the flow.

In chapter 7, we studied the effects of rough walls on bubble drag reduction. In maritime vessels, surfaces which are smooth initially will become rough by means of biofouling and corrosion. Therefore, initially effective air lubrication methods might become ineffective, or even counterproductive. Our experiments showed that roughness on outer cylinder induces strong stable Taylor vortices, i.e. large-scale secondary flow structures. These vortices significantly affect the torque and resulting drag reduction. We showed the existence of two different regimes. i) A regime in which roughness does not induce Taylor rolls. In this regime, the drag reduction is effective. And ii) a regime in which roughness induces strong secondary flows, eliminating any drag reduction.

\section{Outlook}

And now, which further steps should be taken in this line of research? The questions which we posed in the introduction have now been answered as good as I was able to. Of course, many questions remain, and the more one knows about some topic, the more one is aware of how much is not yet known.

We became aware of the existence of a significant gap between laboratory exper- 
iments and the conditions, which are encountered when applying the studied techniques in marine vessels. Vessels are fouled with both calcareous organisms as well as a soft slimy biofilm, which significantly alter the surface of the vessel. Furthermore, large quantities of ions and any other surface active agents are dissolved in (sea)water, thus changing the way dispersed bubbles behave. Clearly, it is impossible to one-toone compare laboratory experiments with distilled water and smooth walls to marine vessels. Therefore, to close this gap it is crucial to mimic more realistic conditions in lab environments.

Numerical tools will become even more important as the computational powers continue to increase. For single-phase flow the gap between DNS and experiments is rapidly closing. Numerically, dealing with multiphase flows, transient effects or wall roughness is difficult, and one could argue that direct numerical simulations in these fields are still in their infancy. LES and RANS simulations, as well as a variety of related and hybrid methods are not limited by computational power, but by physical insight. The practical use of these methods in the fields addressed here is hindered by the way small-scale turbulence is modelled, and more work needs to be done to improve the turbulence models. More collaboration between experimentalists and numericalists will stimulate both fields.

The author would like to add some personal notes on the developments in physics and science. My observation is that the way students are being educated is changing. At highschools, 'iPads' and 'digiboards' largely replaced blackboards and multiplication tables. Given the increasing role computers play, useful tools such as Matlab and Solidworks are given an increasing role in the current curricula at universities. This however comes with a cost, as one can only spend his time once. In my opinion, the theoretical background in physics and mathematics has increasingly come under pressure. Numerical tools, how useful they indeed can be, cannot replace a thorough understanding of physical phenomena and the mathematical description thereof. Given the fact that many universities prefer to focus on 'internationalisation' and 'student-driven learning' rather than on solid physics and math, I think it is clear that a reevaluation of the current curricula has become inevitable. A good education in physical mechanisms is most crucial for the scientific quality of future generations. 


\section{References}

[1] P. Kaluza, A. Közsch, M. T. Gastner, and B. Blasius, "The complex network of global cargo ship movements", J. R. Soc. Interface 7, 1093-1103 (2010).

[2] E. J. Foeth, "Decreasing frictional resistance by air lubrication", in 20th International HISWA Symposium on Yacht Design and Yacht Construction (HISWA) (2008).

[3] D. P. M. van Gils, G. W. Bruggert, D. P. Lathrop, C. Sun, and D. Lohse, "The Twente turbulent Taylor-Couette $\left(T^{3} C\right)$ facility: strongly turbulent (multiphase) flow between independently rotating cylinders", Rev. Sci. Instr. 82, 025105 (2011).

[4] D. P. M. van Gils, S. G. Huisman, G. W. Bruggert, C. Sun, and D. Lohse, "Torque scaling in turbulent Taylor-Couette flow with co- and counter-rotating cylinders", Phys. Rev. Lett. 106, 024502 (2011).

[5] R. Ostilla-Mónico, E. P. van der Poel, R. Verzicco, S. Grossmann, and D. Lohse, "Exploring the phase diagram of fully turbulent Taylor-Couette flow", J. Fluid Mech. 761, 1-26 (2014).

[6] D. P. Lathrop, J. Fineberg, and H. S. Swinney, "Turbulent flow between concentric rotating cylinders at large Reynolds numbers", Phys. Rev. Lett. 68, 1515-1518 (1992).

[7] D. P. Lathrop, J. Fineberg, and H. S. Swinney, "Transition to shear-driven turbulence in Couette-Taylor flow", Phys. Rev. A 46, 6390-6405 (1992).

[8] X. Chavanne, F. Chilla, B. Castaing, B. Hebral, B. Chabaud, and J. Chaussy, "Observation of the ultimate regime in Rayleigh-Bénard convection", Phys. Rev. Lett. 79, 3648 (1997).

[9] D. P. M. van Gils, D. Narezo Guzman, C. Sun, and D. Lohse, "The importance of bubble deformability for strong drag reduction in bubbly turbulent TaylorCouette flow", J. Fluid Mech. 722, 317-347 (2013).

[10] O. Cadot, J. H. Titon, and D. Bonn, "Experimental observation of resonances in modulated turbulence", J. Fluid Mech. 485, 161 (2003). 
[11] T. H. van den Berg, S. Luther, D. P. Lathrop, and D. Lohse, "Drag reduction in bubbly Taylor-Couette turbulence", Phys. Rev. Lett. 94, 044501 (2005).

[12] A. N. Kolmogorov, "The local structure of turbulence in incompressible viscous fluid for very large Reynolds numbers", Dokl. Akad. Nauk SSSR 30, 9-13 (1941), reprinted in Proc. R. Soc. Lond. A 434, 9-13 (1991).

[13] S. B. Pope, Turbulent Flow (Cambridge University Press, Cambridge) (2000).

[14] A. N. Kolmogorov, "On degeneration (decay) of isotropic turbulence in incompressible viscous liquid", Dokl. Akad. Nauk SSSR 31, 538-540 (1941).

[15] A. N. Kolmogorov, "Dissipation of energy in locally isotropic turbulence", Dokl. Akad. Nauk SSSR 32, 16-18 (1941), reprinted in Proc. R. Soc. Lond. A 434, 15-17 (1991).

[16] P. G. Saffman, "Note on decay of homogeneous turbulence", Phys. Fluids 10, 1349 (1967).

[17] K. R. Sreenivasan, "On the scaling of the turbulence energy dissipation rate", Phys. Fluids 27, 1048 (1984).

[18] J. C. Vassilicos, "Dissipation in Turbulent Flows", Annu. Rev. Fluid Mech. 47, 95-114 (2015).

[19] M. Sinhuber, E. Bodenschatz, and G. P. Bewley, "Decay of Turbulence at High Reynolds Numbers", Phys. Rev. Lett. 114, 034501 (2015).

[20] W. K. George, "The decay of homogeneous isotropic turbulence", Phys. Fluids A 4, 1492 (1992).

[21] M. Smith, R. J. Donnelly, N. Goldenfeld, and W. F. Vinen, "Decay of vorticity in homogeneous turbulence", Phys. Rev. Lett. 71, 2583 (1993).

[22] W. K. George and H. Wang, "The exponential decay of homogeneous turbulence", Phys. Fluids 21, 025108 (2009).

[23] S. R. Stalp, L. Skrbek, and R. J. Donnelly, "Decay of grid turbulence in a finite channel", Phys. Rev. Lett. 82, 4381-4384 (1999).

[24] L. Skrbek and S. R. Stalp, "On the decay of homogeneous isotropic turbulence", Phys. Fluids 12, 1997-2019 (2000).

[25] P. Burattini, P. Lavoie, and R. A. Antonia, "On the normalized turbulent energy dissipation rate", Phys. Fluids 17, 1-4 (2005).

[26] A. Thormann and C. Meneveau, "Decay of homogeneous, nearly isotropic turbulence behind active fractal grids", Phys. Fluids 26, 025112 (2014). 
[27] L. Biferale, G. Boffetta, A. Celani, A. Lanotte, F. Toschi, and M. Vergassola, "The decay of homogeneous anisotropic turbulence", Phys. Fluids 15, 21052112 (2003).

[28] P. Burattini, P. Lavoie, A. Agrawal, L. Djenidi, and R. A. Antonia, "Power law of decaying homogeneous isotropic turbulence at low Reynolds number", Phys. Rev. E 73, 066304 (2006).

[29] T. Ishida, P. A. Davidson, and Y. Kaneda, "On the decay of isotropic turbulence", J. Fluid Mech. 564, 455-475 (2006).

[30] T. Teitelbaum and P. D. Mininni, "Effect of helicity and rotation on the free decay of turbulent flows", Phys. Rev. Lett. 103, 014501 (2009).

[31] P. Lavoie, L. Djenidi, and R. A. Antonia, "Effects of initial conditions in decaying turbulence generated by passive grids", J. Fluid Mech. 585, 395-420 (2007).

[32] P. C. Valente and J. C. Vassilicos, "The decay of turbulence generated by a class of multiscale grids", J. Fluid Mech. 687, 300-340 (2011).

[33] D. Hurst and J. C. Vassilicos, "Scalings and decay of fractal-generated turbulence", Phys. Fluids 19, 035103 (2007).

[34] R. A. Antonia, R. J. Smalley, T. Zhou, F. Anselmet, and L. Danaila, "Similarity of energy structure functions in decaying homogeneous isotropic turbulence", J. Fluid Mech. 487, 245-269 (2003).

[35] G. L. Eyink and D. J. Thompson, "Free decay of grid turbulence in a finite channel", Phys. Fluids 12, 477-478 (2000).

[36] M. Meldi, P. Sagaut, and D. Lucor, "A stochastic view of isotropic turbulence decay", J. Fluid Mech. 668, 351-362 (2011).

[37] G. Riboux, F. Risso, and D. Legendre, "Experimental characterization of the agitation generated by bubbles rising at high Reynolds number", J. Fluid Mech. 643, 509 (2010).

[38] P. A. Davidson, "The minimum energy decay rate in quasi-isotropic grid turbulence", Phys. Fluids 23, 085108 (2011).

[39] L. D. Landau and E. M. Lifshitz, Fluid Mechanics (Pergamon Press, Oxford) (1987).

[40] D. Lohse, "Crossover from high to low Reynolds number turbulence", Phys. Rev. Lett. 73, 3223-3226 (1994).

[41] H. Effinger and S. Grossmann, "Static structure function of turbulent flow from the Navier-Stokes equation", Z. Phys. B 66, 289 (1987). 
[42] H. Touil, J.-P. Bertoglio, and L. Shao, "The decay of turbulence in a bounded domain", J. Turbul. 3, N49 (2002).

[43] A. J. Smits, B. J. McKeon, and I. Marusic, "High-Reynolds number wall turbulence", Ann. Rev. Fluid Mech. 43, 353-375 (2011).

[44] M. Hultmark, M. Vallikivi, S. C. C. Bailey, and A. J. Smits, "Turbulent pipe flow at extreme Reynolds numbers", Phys. Rev. Lett. 108, 094501 (2012).

[45] I. Marusic, B. J. McKeon, P. A. Monkewitz, H. M. Nagib, A. J. Smits, and K. R. Sreenivasan, "Wall-bounded turbulent flows at high Reynolds numbers: Recent advances and key issues", Phys. Fluids 22, 065103 (2010).

[46] A. J. Smits and I. Marusic, "Wall-bounded turbulence", Phys. Today 66, 25-30 (2013).

[47] G. Ahlers, S. Grossmann, and D. Lohse, "Heat transfer and large scale dynamics in turbulent Rayleigh-Bénard convection", Rev. Mod. Phys. 81, 503 (2009).

[48] D. Lohse and K.-Q. Xia, "Small-scale properties of turbulent Rayleigh-Bénard convection", Ann. Rev. Fluid Mech. 42, 335-364 (2010).

[49] R. J. Donnelly, "Taylor-Couette flow: the early days", Phys. Today 44(11), 32-39 (1991).

[50] M. A. Fardin, C. Perge, and N. Taberlet, " "The hydrogen atom of fluid dynamics" - Introduction to the Taylor-Couette flow for Soft Matter scientists", Soft Matter 10, 3523 (2014).

[51] S. Grossmann, D. Lohse, and C. Sun, "High Reynolds number Taylor-Couette turbulence", Ann. Rev. Fluid Mech. 48, 53 (2016).

[52] S. G. Huisman, R. C. A. van der Veen, C. Sun, and D. Lohse, "Multiple states in highly turbulent Taylor-Couette flow", Nat. Commun. 5, 3820 (2014).

[53] G. S. Lewis and H. L. Swinney, "Velocity structure functions, scaling, and transitions in high-Reynolds-number Couette-Taylor flow", Phys. Rev. E 59, 5457-5467 (1999).

[54] S. G. Huisman, S. Scharnowski, C. Cierpka, C. Kähler, D. Lohse, and C. Sun, "Logarithmic boundary layers in strong Taylor-Couette turbulence", Phys. Rev. Lett. 110, 264501 (2013).

[55] R. Ostilla-Mónico, E. P. van der Poel, R. Verzicco, S. Grossmann, and D. Lohse, "Boundary layer dynamics at the transition between the classical and the ultimate regime of Taylor-Couette flow", Phys. Fluids 26, 015114 (2014).

[56] D. Borrero-Echeverry, M. F. Schatz, and R. Tagg, "Transient turbulence in Taylor-Couette flow", Phys. Rev. E 81, 025301 (2010). 
[57] R. Ostilla-Mónico, R. Verzicco, S. Grossmann, and D. Lohse, "Turbulence decay towards the linearly stable regime of Taylor-Couette flow", J. Fluid Mech. 748, R3 (2014).

[58] S. G. Huisman, D. P. M. Van Gils, and C. Sun, "Applying laser Doppler anemometry inside a Taylor-Couette geometry using a ray-tracer to correct for curvature effects", Eur. J. Mech. B/Fluids 36, 115-119 (2012).

[59] R. Mei, "Velocity fidelity of flow tracer particles", Exp. Fluids 22, 1-13 (1996).

[60] C. D. Andereck, S. S. Liu, and H. L. Swinney, "Flow regimes in a circular Couette system with independently rotating cylinders", J. Fluid Mech. 164, 155 (1986).

[61] M. V. Zagarola and A. J. Smits, "Mean-flow scaling of turbulent pipe flow", J. Fluid Mech. 373, 33-79 (1998).

[62] D. Lohse, "Periodically kicked turbulence", Phys. Rev. E 62, 4946 (2000).

[63] A. von der Heydt, S. Grossmann, and D. Lohse, "Resonances in modulated turbulence", Phys. Rev. E 67, 046308 (2003).

[64] J. O. Hooghoudt, D. Lohse, and F. Toschi, "Decaying and kicked turbulence in a shell model", Phys. Fluids 13, 2013 (2001).

[65] A. von der Heydt, S. Grossmann, and D. Lohse, "Resonances in modulated turbulence. ii. numerical simulations", Phys. Rev. E 68, 066302 (2003).

[66] P. E. Hamlington and W. J. A. Dahm, "Frequency response of periodically sheared homogeneous turbulence", Phys. Fluids 21, 055107 (2009).

[67] D. Yu and S. S. Girimaji, "Direct numerical simulation of homogeneous turbulence subject to periodic shear", J. Fluid Mech. 566, 117-151 (2006).

[68] A. K. Kuczaj, B. J. Geurts, and D. Lohse, "Response maxima in time-modulated turbulence: Direct numerical simulations", Europhys. Lett. 73, 851 (2006).

[69] A. K. Kuczaj, B. J. Geurts, D. Lohse, and W. van de Water, "Turbulence modification by periodically modulated scale-dependent forcing", Comput. Fluids $\mathbf{3 7 ,} 816$ (2008).

[70] H. E. Cekli, C. Tipton, and W. Van De Water, "Resonant enhancement of turbulent energy dissipation", Phys. Rev. Lett. 105, 044503 (2010).

[71] J. R. Womersley, "Method for the calculation of velocity rate of flow and viscous drag in arteries when the pressure gradient is known", J. Physiol. 127, 553-572 (1955). 
[72] L. Shemer, I. Wygnanski, and E. Kit, "Pulsating flow in a pipe", J. Fluid Mech. 153, 313-337 (1985).

[73] Z.-X. Mao and T. J. Hanratty, "Studies of the wall shear stress in a turbulent pulsating pipe flow", J. Fluid Mech. 170, 545-564 (1986).

[74] C. R. Lodahl, B. M. Sumer, and J. Fredsøe, "Turbulent combined oscillatory flow and current in a pipe", J. Fluid Mech. 373 (1998).

[75] S. He and S. D. Jackson, "An experimental study of pulsating turbulent flow in a pipe", Eur. J. Mech. B/Fluids 28, 309-320 (2009).

[76] M. Manna, A. Vacca, and R. Verzicco, "Pulsating pipe flow with large-amplitude oscillations in the very high frequency regime. part 1. time-averaged analysis", J. Fluid Mech. 700, 246-282 (2012).

[77] T. Sarpkaya, "Experimental determination of the critical Reynolds number for pulsating Poiseuille flow", Trans. ASME: J. Basic. Eng. 88, 589-598 (1966).

[78] E. L. Yellin, "Laminar-turbulent transition process in pulsatile flow", Circulat. Res. 19, 791-804 (1966).

[79] B. R. Ramaprian and S. W. Tu, "An experimental study of oscillatory pipe flow at transitional Reynolds number", J. Fluid Mech. 100, 513-544 (1980).

[80] X.-L. Jin and K.-Q. Xia, "An experimental study of kicked thermal turbulence", J. Fluid Mech. 606, 133 (2008).

[81] S. Sterl, H.-M. Li, and J.-Q. Zhong, "Dynamical and statistical phenomena of circulation and heat transfer in periodically forced rotating turbulent RayleighBenard convection", Phys. Rev. Fluids 1, 084401 (2016).

[82] C.-C. Chien, D. B. Blum, and G. A. Voth, "Effects of fluctuation energy input on the small scales in turbulence", J. Fluid Mech. 737, 527-551 (2013).

[83] C. Colebrook, "Turbulent flow in pipes, with particular reference to the transitional region between smooth and rough wall laws", J. Inst. Chem. Eng. 11, 113 (1939).

[84] L. F. Moody, "Friction factors for pipe flow", Trans. ASME 66, 671-684 (1944).

[85] M. P. Schultz, "Effects of coating roughness and biofouling on ship resistance and powering", Biofouling 23, 331-341 (2007).

[86] M. P. Schultz and K. A. Flack, "The rough-wall turbulent boundary layer from the hydraulically smooth to the fully rough regime", J. Fluid Mech. 580, 381405 (2007). 
[87] M. Thakkar, A. Busse, and N. D. Sandham, "Direct numerical simulation of turbulent channel flow over a surrogate for Nikuradse-type roughness", J. Fluid Mech. 837, R1 (2017).

[88] M. MacDonald, L. Chan, D. Chung, N. Hutchins, and A. Ooi, "Turbulent flow over transitionally rough surfaces with varying roughness densities", J. Fluid Mech. 804, 130-161 (2016).

[89] M. A. Shockling, J. J. Allen, and A. J. Smits, "Roughness effects in turbulent pipe flow", J. Fluid Mech. 564, 267-285 (2006).

[90] H. Ren and W. Yanhua, "Turbulent boundary layers over smooth and rough forward-facing steps", Phys. Fluids 23, 045102 (2011).

[91] A. Busse, M. Lützner, and N. D. Sandham, "Direct numerical simulation of turbulent flow over a rough surface based on a surface scan", Computers \& Fluids 116, 129-147 (2015).

[92] K. A. Flack, "Moving beyond Moody", J. Fluid Mech. 842, 1-4 (2018).

[93] V. Spandan, R. Verzicco, and D. Lohse, "Deformable ellipsoidal bubbles in Taylor-Couette flow with enhanced Euler-Lagrangian tracking", Phys. Rev. Fluids 2, 104304 (2017).

[94] F. H. Busse, "Viewpoint: The twins of turbulence research", Physics 5, 4 (2012).

[95] B. Eckhardt, S. Grossmann, and D. Lohse, "Scaling of global momentum transport in Taylor-Couette and pipe flow", Eur. Phys. J. B 18, 541-544 (2000).

[96] O. Cadot, Y. Couder, A. Daerr, S. Douady, and A. Tsinober, "Energy injection in closed turbulent flows: Stirring through boundary layers versus inertial stirring", Phys. Rev. E 56, 427-433 (1997).

[97] G. Ndongo Fokoua, C. Gabillet, A. Aubert, and C. Colin, "Effect of bubble's arrangement on the viscous torque in bubbly Taylor-Couette flow", Phys. Fluids 27, 034105 (2015).

[98] M. S. Paoletti and D. P. Lathrop, "Angular momentum transport in turbulent flow between independently rotating cylinders", Phys. Rev. Lett. 106, 024501 (2011).

[99] S. Maretzke, B. Hof, and M. Avila, "Transient growth in linearly stable TaylorCouette flows", J. Fluid Mech. 742, 254-290 (2014).

[100] R. L. Webb, E. R. G. Eckert, and R. J. Goldstein, "Heat transfer and friction in tubes with repeated-rib roughness", Int. J. Heat Mass Transfer 14, 601-617 (1971). 
[101] Y. Kodama, A. Kakugawa, T. Takahashi, and H. Kawashima, "Experimental studies on microbubbles and their applicability to ships for skin friction reduction", Int. J. Heat and Fluid Flow 21, 582-588 (2000).

[102] T. Takahashi, A. Kakugawa, S. Nagaya, T. Yanagihara, and Y. Kodama, "Mechanisms and scale effects of skin friction reduction by microbubbles.", in Proceedings of 2nd symposium on smart control of turbulence (Univ. of Tokyo, Japan) (2001).

[103] R. A. Verschoof, D. Bakhuis, P. A. Bullee, S. G. Huisman, C. Sun, and D. Lohse, "Air cavities at the inner cylinder of turbulent taylor-couette flow", Int. J. Mulitphase Flow submitted (2018).

[104] M. P. Schultz, J. A. Bendick, E. R. Holm, and W. M. Hertel, "Economic impact of biofouling on a naval surface ship", Biofouling 27, 87-98 (2011).

[105] E. S. Winkel, S. L. Ceccio, D. R. Dowling, and M. Perlin, "Bubble-size distributions produced by wall injection of air into flowing freshwater, saltwater and surfactant solutions", Exp. Fluids 37, 802-810 (2004).

[106] X. Shen, M. Perlin, and S. L. Ceccio, "Influence of bubble size on micro-bubble drag reduction", Exp. Fluids 41, 415-424 (2006).

[107] R. C. A. van der Veen, S. G. Huisman, O.-Y. Dung, H.-L. Tang, C. Sun, and D. Lohse, "Exploring the phase space of multiple states in highly turbulent taylor-couette flow", Phys. Rev. Fluids 1, 024401 (2016).

[108] X. Zhu, R. Ostilla-Monico, R. Verzicco, and D. Lohse, "Direct numerical simulation of Taylor-Couette flow with grooved walls: torque scaling and flow structure", J. Fluid Mech. 794, 746-774 (2016).

[109] S. G. Huisman, D. Lohse, and C. Sun, "Statistics of turbulent fluctuations in counter-rotating Taylor-Couette flows", Phys. Rev. E 88, 063001 (2013).

[110] S. F. Tardu, G. Binder, and R. F. Blackwelder, "Turbulent channel flow with large-amplitude velocity oscillations", J. Fluid Mech. 267, 109-151 (1994).

[111] G. J. Brereton, W. C. Reynolds, and R. Jayaraman, "Response of a turbulent boundary layer to sinusoidal free-stream unsteadiness", J. Fluid Mech. 221, 131-159 (1990).

[112] V. Spandan, R. Ostilla-Mónico, R. Verzicco, and D. Lohse, "Drag reduction in numerical two-phase Taylor-Couette turbulence using an Euler-Lagrange approach", J. Fluid. Mech. 798, 411-435 (2016).

[113] G. M. Rotte, O. Zverkhovskyi, M. Kerkvliet, and T. J. C. van Terwisga, "On the physical mechanisms for the numerical modelling of flows around air lubricated ships", in Proc. 12th Int. Conf. on Hydrodynamics (ICHD) (2016). 
[114] B. van Ruymbeke, Y. Murai, Y. Tasaka, Y. Oishi, C. Gabillet, and C. Colin, "Quantitative visualization of swirl and cloud bubbles in Taylor-Couette flow", J. Visualization 20, 349 (2017).

[115] K. Sugiyama, E. Calzavarini, and D. Lohse, "Microbubble drag reduction in Taylor-Couette flow in the wavy vortex regime", J. Fluid Mech. 608, 21-41 (2008).

[116] Y. Murai, H. Oiwa, and Y. Takeda, "Bubble behavior in a vertical TaylorCouette flow", J. Phys. (Conf. Series) 14, 143-156 (2005).

[117] Y. Murai, H. Oiwa, and Y. Takeda, "Frictional drag reduction in bubbly Couette-Taylor flow", Phys. Fluids 20, 034101 (2008).

[118] G. K. El Khoury, P. Schlatter, A. Noorani, P. F. Fischer, G. Brethouwer, and A. V. Johansson, "Direct numerical simulation of turbulent pipe flow at moderately high Reynolds numbers", Flow Turbul. Combust. 91, 475-495 (2013).

[119] R. Ostilla-Mónico, R. Verzicco, and D. Lohse, "Effects of the computational domain size on direct numerical simulations of Taylor-Couette turbulence with stationary outer cylinder", Phys. Fluids 27, 025110 (2015).

[120] M. Avila, "Stability and angular-momentum transport of fluid flows between co-rotating cylinders", Phys. Rev. Lett. 108, 124501 (2012).

[121] E. P. van der Poel, R. Ostilla-Mónico, J. Donners, and R. Verzicco, "A pencil distributed finite difference code for strongly turbulent wall-bounded flows", Computers \& Fluids 116, 10-16 (2015).

[122] J. Yang and E. Balaras, "An embedded-boundary formulation for large-eddy simulation of turbulent flows interacting with moving boundaries", J. Comput. Phys. 215, 12-40 (2006).

[123] E. A. Fadlun, R. Verzicco, P. Orlandi, and J. Mohd-Yusof, "Combined immersed-boundary finite-difference methods for three-dimensional complex flow simulations", J. Comput. Phys. 161, 35-60 (2000).

[124] R. Verzicco and P. Orlandi, "A finite-difference scheme for three-dimensional incompressible flow in cylindrical coordinates", J. Comput. Phys. 123, 402-413 (1996).

[125] T. H. van den Berg, C. R. Doering, D. Lohse, and D. P. Lathrop, "Smooth and rough boundaries in turbulent Taylor-Couette flow", Phys. Rev. E 68, 036307 (2003).

[126] R. A. Verschoof, R. C. A. van der Veen, C. Sun, and D. Lohse, "Bubble drag reduction requires large bubbles", Phys. Rev. Lett. 117, 104502 (2016). 
[127] D. Saranadhi, D. Chen, J. A. Kleingartner, S. Srinivasan, R. B. Cohen, and G. H. McKinley, "Sustained drag reduction in a turbulent flow using a low temperature Leidenfrost surface", Science Advances 2, E1600686 (2016).

[128] S. Srinivasan, J. A. Kleingartner, J. B. Gilbert, R. B. Cohen, A. J. B. Milne, and G. H. McKinley, "Sustainable drag reduction in turbulent Taylor-Couette flows by depositing srayable superhydrophobic surfaces", Phys. Rev. Lett. 114, 014501 (2015).

[129] B. J. Rosenberg, T. van Buren, K. F. Matthew, and A. J. Smits, "Turbulent drag reduction over air- and liquid- impregnated surfaces", Phys. Fluids 28, 015103 (2016).

[130] K. I. Matveev, "On the limiting parameters of artificial cavitation", Ocean Eng. 30, 1179-1190 (2003).

[131] K. I. Matveev, "Applications of artificial cavitation for reducing ship drag", Ocean Eng. Int. 9, 35-41 (2005).

[132] A. A. Butuzov, "Artificial cavitation flow behind a slender wedge on the lower surface of a horizontal wall", Fluid Dyn. 2, 56-58 (1967).

[133] R. Latorre, "Ship hull drag reduction using bottom air injection", Ocean Eng. 24, 161-175 (1997).

[134] O. Zverkhovskyi, "Ship drag reduction by air cavities", Ph.D. thesis, Delft University of Technology, Delft, NL (2014).

[135] E. Amromin, G. Karafiath, and B. Metcalf, "Ship drag reduction by air bottom ventilated cavitation in calm water and waves", J. Ship Research 55, 196-207 (2011).

[136] C. Hoang, Y. Toda, and Y. Sanada, "Full scale experiment for frictional resistance reduction using air lubrication method", in Proc. of the 19th International Offshore and Polar Engineering Conference, 812-817 (ISOPE) (2009).

[137] S. Mizokami, C. Kawakita, Y. Kodan, S. Takano, S. Higasa, and R. Shigenaga, "Experimental study of air lubrication method and verification of effects on actual hull by means of sea trial", Mitsubishi Heavy Industries Technical Review 47, 41-47 (2010).

[138] S. Mäkiharju, M. Perlin, and S. L. Ceccio, "On the energy economics of air lubrication drag reduction", Int. J. of Naval Architecture in Oceanic Eng. 4, 412-422 (2012).

[139] K. A. Lay, R. Yakushiji, S. Mäkiharju, M. Perlin, and S. L. Ceccio, "Partial cavity drag reduction at high Reynolds numbers", J. Ship Research 52, 109-119 (2010). 
[140] S. A. Mäkiharju, B. R. Elbing, A. Wiggins, S. Schinasi, J.-M. Vanden-Broeck, M. Perlin, D. R. Dowling, and S. L. Ceccio, "On the scaling of air entrainment from a ventilated partial cavity", J. Fluid Mech. 732 (2013).

[141] E. Climent, M. Simonnet, and J. Magnaudet, "Preferential accumulation of bubbles in Couette-Taylor flow patterns", Phys. Fluids 19, 083301 (2007).

[142] H. Djeridi, C. Gabillet, and J. Y. Billard, "Two-phase Couette-Taylor flow: Arrangement and affects on the flow structures", Phys. Fluids 16, 128 (2004).

[143] L. Larsson and H. C. Raven, The principles of naval architecture series: ship resistance and flow (The Society of Naval Architects and Marine Engineers, New York) (2010).

[144] R. A. Verschoof, S. G. Huisman, R. C. A. van der Veen, C. Sun, and D. Lohse, "Self-similar decay of high Reynolds number Taylor-Couette turbulence", Phys. Rev. Fluids 1, 062402(R) (2016).

[145] J. Nikuradse, "Strömungsgesetze in rauhen Rohren", Forschungsheft Arb. Ing.Wes. 361 (1933).

[146] M. Hultmark, M. Vallikivi, S. C. C. Bailey, and A. J. Smits, "Logarithmic scaling of turbulence in smooth-and rough-wall pipe flow", J. Fluid Mech. 728, 376-395 (2013).

[147] L. Chan, M. MacDonald, D. Chung, N. Hutchins, and A. Ooi, "A systematic investigation of roughness height and wavelength in turbulent pipe flow in the transitionally rough regime", J. Fluid Mech. 771, 743-777 (2015).

[148] D. Chung, L. Chan, M. MacDonald, N. Hutchins, and A. Ooi, "A fast direct numerical simulation method for characterising hydraulic roughness", J. Fluid Mech. 773, 418-431 (2015).

[149] D. T. Squire, C. Morrill-Winter, N. Hutchins, M. P. Schultz, J. C. Klewicki, and I. Marusic, "Comparison of turbulent boundary layers over smooth and rough surfaces up to high Reynolds numbers", J. Fluid Mech. 795, 210-240 (2016).

[150] J. Jiménez, "Turbulent flows of rough walls", Ann. Rev. Fluid Mech. 36, 173196 (2004).

[151] K. A. Flack and M. P. Schultz, "Roughness effects on wall-bounded turbulent flows", Phys. Fluids 26, 101305 (2014).

[152] H. Schlichting and K. Gersten, Boundary layer theory, 8th edition (Springer Verlag, Berlin) (2000).

[153] X. He, D. Funfschilling, E. Bodenschatz, and G. Ahlers, "Heat transport by turbulent Rayleigh-Bénard convection for $\operatorname{Pr}=0.8$ and $4 \times 10^{11}<R a<$ $2 \times 10^{14}$ : ultimate-state transition for aspect ratio $\Gamma=1.00$ ", New J. Phys.. 14, 063030 (2012). 
[154] X. He, D. Funfschilling, H. Nobach, E. Bodenschatz, and G. Ahlers, "Transition to the ultimate state of turbulent Rayleigh-Bénard convection", Phys. Rev. Lett. 108, 024502 (2012).

[155] R. Ostilla-Mónico, R. Verzicco, S. Grossmann, and D. Lohse, "The near-wall region of highly turbulent Taylor-Couette flow", J. Fluid Mech. 788, 95-117 (2016).

[156] C. Doering and P. Constantin, "Variational bounds on energy dissipation in incompressible flows: III. Convection", Phys. Rev. E 53, 5957-5981 (1996).

[157] R. Nicodemus, S. Grossmann, and M. Holthaus, "Variational bound on energy dissipation in turbulent shear flow", Phys. Rev. Lett. 79, 4170 (1997).

[158] S. C. Plasting and R. R. Kerswell, "Improved upper bound on the energy dissipation rate in plane Couette flow: the full solution to Busse's problem and the Constantin-Doering-Hopf problem with one-dimensional background field", J. Fluid Mech. 477, 363-379 (2003).

[159] S. Toppaladoddi, S. Succi, and J. S. Wettlaufer, "Roughness as a route to the ultimate regime of thermal convection", Phys. Rev. Lett. 118, 074503 (2017).

[160] Y.-C. Xie and K.-Q. Xia, "Turbulent thermal convection over rough plates with varying roughness geometries", J. Fluid Mech. 825, 573-599 (2017).

[161] X. Zhu, R. A. J. M. Stevens, R. Verzicco, and D. Lohse, "Roughness-facilitated local $1 / 2$ scaling does not imply the onset of the ultimate regime of thermal convection", Phys. Rev. Lett. 119, 154501 (2017).

[162] D. Lohse and F. Toschi, "The ultimate state of thermal convection", Phys. Rev. Lett. 90, 034502 (2003).

[163] M. Gibert, H. Pabiou, F. Chilla, and B. Castaing, "High-Rayleigh-number convection in a vertical channel", Phys. Rev. Lett. 96, 084501 (2006).

[164] M. Cholemari and J. Arakeri, "Axially homogeneous, zero mean flow buoyancydriven turbulence in a vertical pipe", J. Fluid Mech. 621, 69-102 (2009).

[165] T. von Kármán, "Über laminare und turbulente Reibung", Z. angew. Math. Mech. 1, 233-252 (1921).

[166] Y. Shen, P. Tong, and K.-Q. Xia, "Turbulent convection over rough surfaces", Phys. Rev. Lett. 76, 908-911 (1996).

[167] Y. B. Du and P. Tong, "Turbulent thermal convection in a cell with ordered rough boundaries", J. Fluid Mech. 407, 57-84 (2000).

[168] P. E. Roche, B. Castaing, B. Chabaud, and B. Hebral, "Observation of the 1/2 power law in Rayleigh-Bénard convection", Phys. Rev. E 63, 045303 (2001). 
[169] J. C. Tisserand, M. Creyssels, Y. Gasteuil, H. Pabiou, M. Gibert, B. Castaing, and F. Chilla, "Comparison between rough and smooth plates within the same Rayleigh-Benard cell", Phys. Fluids 23, 015105 (2011).

[170] P. Wei, T.-S. Chan, R. Ni, X.-Z. Zhao, and K.-Q. Xia, "Heat transport properties of plates with smooth and rough surfaces in turbulent thermal convection", J. Fluid Mech. 740, 28-46 (2014).

[171] H. J. Brauckmann and B. Eckhardt, "Direct numerical simulations of local and global torque in Taylor-Couette flow up to Re $=30$ 000", J. Fluid Mech. 718, 398-427 (2013).

[172] S. Grossmann, D. Lohse, and C. Sun, "Velocity profiles in strongly turbulent Taylor-Couette flow", Phys. Fluids 26, 025114 (2014).

[173] X. Zhu, R. Verzicco, and D. Lohse, "Disentangling the origins of torque enhancement through wall roughness in Taylor-Couette turbulence", J. Fluid Mech. 812, 279-293 (2017).

[174] G. I. Taylor, "Stability of a viscous liquid contained between two rotating cylinders", Phil. Trans. R. Soc. A 223, 289-343 (1923).

[175] H. J. Brauckmann and B. Eckhardt, "Intermittent boundary layers and torque maxima in Taylor-Couette flow", Phys. Rev. E 87, 033004 (2013).

[176] D. P. M. van Gils, S. G. Huisman, S. Grossmann, C. Sun, and D. Lohse, "Optimal Taylor-Couette turbulence", J. Fluid Mech. 706, 118-149 (2012).

[177] A. Chouippe, E. Climent, D. Legendre, and C. Gabillet, "Numerical simulation of bubble dispersion in turbulent Taylor-Couette flow", Phys. Fluids 26, 043304 (2014).

[178] B. Martínez-Arias, J. Peixinho, O. Crumeyrolle, and I. Mutabazi, "Effect of the number of vortices on the torque scaling in Taylor-Couette flow", J. Fluid Mech. 748, 756-767 (2014).

[179] S. Grossmann and D. Lohse, "Scaling in thermal convection: A unifying view", J. Fluid. Mech. 407, 27-56 (2000).

[180] S. Grossmann and D. Lohse, "Thermal convection for large Prandtl number", Phys. Rev. Lett. 86, 3316-3319 (2001).

[181] X. D. Shang, P. Tong, and K.-Q. Xia, "Scaling of the local convective heat flux in turbulent Rayleigh-Bénard convection", Phys. Rev. Lett. 100, 244503 (2008).

[182] R. Ni, S.-D. Huang, and K.-Q. Xia, "Local energy dissipation rate balances local heat flux in the center of turbulent thermal convection", Phys. Rev. Lett. 107, 174503 (2011). 
[183] T. J. Walsh and R. J. Donnelly, "Taylor-Couette flow with periodically corotated and counterrotated cylinders", Phys. Rev. Lett. 60, 700 (1988).

[184] R. Ostilla-Mónico, X. Zhu, V. S. Spandan, R. Verzicco, and D. Lohse, "Three life stages of wall-bounded decay of Taylor-Couette turbulence", Phys. Rev. Fluids 2, 114601 (2017).

[185] R. H. Kraichnan, "Turbulent thermal convection at arbritrary Prandtl number", Phys. Fluids 5, 1374 (1962).

[186] S. Grossmann and D. Lohse, "Multiple scaling in the ultimate regime of thermal convection", Phys. Fluids 23, 045108 (2011).

[187] A. Ganske, T. Gebhardt, and S. Grossmann, "Taylor-Couette flow with time modulated inner cylinder velocity", Phys. Lett. A 192 (1994).

[188] A. Ganske, T. Gebhardt, and S. Grossmann, "Modulation effects along stability border in Taylor-Couette flow", Phys. Fluids 6, 12 (1994).

[189] B. Eckhardt, S. Grossmann, and D. Lohse, "Torque scaling in turbulent TaylorCouette flow between independently rotating cylinders", J. Fluid Mech. 581, 221-250 (2007).

[190] W. Bos, T. T. Clark, and R. Rubinstein, "Small scale response and modeling of periodically forced turbulence", Phys. Fluids 19, 055107 (2007).

[191] C. F. Barenghi and C. A. Jones, "Modulated Taylor-Couette flow", J. Fluid Mech. 208, 127-160 (1989).

[192] G. Ahlers, "Effect of time-periodic modulation of the driving on Taylor-vortex flow", Bull. Am. Phys. Soc. 32, 2068 (1987).

[193] A. Kitagawa, K. Hishida, and Y. Kodama, "Flow structure of microbubbleladen turbulent channel flow measured by PIV combined with the shadow image technique", Experiments in Fluids 38, 466-475 (2005).

[194] S. Takagi and Y. Matsumoto, "Surfactant effects on bubble motion and bubbly flows", Annu. Rev. Fluid Mech. 43, 615-636 (2011).

[195] N. K. Madavan, S. Deutsch, and C. L. Merkle, "Reduction of turbulent skin friction by micro-bubbles", Phys. Fluids 27, 356 (1984).

[196] S. Deutsch, M. Moeny, A. A. Fontaine, and H. Petrie, "Microbubble drag reduction in rough walled turbulent boundary layers with comparison against polymer drag reduction", Exp. in Fluids 37, 731-744 (2004).

[197] M. Muradoglu and G. Tryggvason, "Simulations of soluble surfactants in 3d multiphase flow", J. Comp. Phys. 274, 737-757 (2014). 
[198] V. S. J. Craig, "Bubble coalescence and specific-ion effects", Current Opinion in Colloid and Interface Sci. 9, 178-184 (2004).

[199] S. F. Wong, J. S. Lim, and S. S. Dol, "Crude oil emulsion: A review on formation, classification and stability of water-in-oil emulsions", J. Petrol. Sci. Eng. 135, 498-504 (2015).

[200] W. C. Ikealumba and H. Wu, "Some recent advances in liquefied natural gas (lng) production, spill, dispersion, and safety", Energy Fuels 28, 3556-3586 (2014).

[201] J. Magnaudet and I. Eames, "The motion of high-Reynolds number bubbles in inhomogeneous flows", Annu. Rev. Fluid Mech. 32, 659-708 (2000).

[202] J. G. Göbel and G. R. Joppien, "Dynamic interfacial tensions of aqueous triton x-100 solutions in contact with air, cyclohexane, n-heptane, and n-hexadecane", J. Coll. Interf. Sci. 191, 30-37 (1997).

[203] S. Takagi, T. Ogasawara, and Y. Matsumoto, "The effects of surfactant on the multiscale structure of bubbly flows.", Philos. Trans. A. Math. Phys. Eng. Sci. 366, 2117-2129 (2008).

[204] A. Einstein, "Eine neue Bestimmung der Moleküldimensionen", Ann. Phys. 19, 289-306 (1906).

[205] J. C. Lu, A. Fernandez, and G. Tryggvason, "Drag reduction in a turbulent channel due to bubble injection", Phys. Fluids 17, 095102 (2005).

[206] A. Ferrante and S. Elghobashi, "On the physical mechanisms of drag reduction in a spatially-developing turbulent boundary layer laden with microbubbles", J. Fluid Mech. 503, 345 (2004).

[207] C. Merkle and S. Deutsch, "Microbubble drag reduction", in Frontiers in Experimental Fluid Mechanics - Lecture notes in Engineering, Vol. 46, edited by M. G. el Hak, 291 (Springer, Berlin) (1989).

[208] B. R. Elbing, S. Mäkiharju, A. Wiggins, M. Perlin, D. R. Dowling, and S. L. Ceccio, "On the scaling of air layer drag reduction", J. Fluid Mech. 717, 484$513(2013)$.

[209] B. R. Elbing, E. S. Winkel, K. A. Lay, S. L. Ceccio, D. R. Dowling, and M. Perlin, "Bubble-induced skin-friction drag reduction and the abrupt transition to air-layer drag reduction", J. Fluid Mech. 612, 201-236 (2008).

[210] T. H. van den Berg, D. P. M. van Gils, D. P. Lathrop, and D. Lohse, "Bubbly turbulent drag reduction is a boundary layer effect", Phys. Rev. Lett. 98, 084501 (2007). 
[211] K. Sugiyama, T. Kawamura, S. Takagi, and Y. Matsumoto, "The Reynolds number effect on the microbubble drag reduction", in Proceedings of the 5th Symposium on Smart Conrol of Turbulence, 31-43 (Japan) (2004).

[212] S. Deutsch, A. A. Fontaine, M. J. Moeny, and H. Petrie, "Combined polymer and microbubble drag reduction on a large flat plate", J. Fluid Mech. 556, 309-327 (2006).

[213] I. Kumagai, Y. Takahashi, and Y. Murai, "Power-saving device for air bubble generation using a hydrofoil to reduce ship drag: Theory, experiments, and application to ships", Ocean Eng. 95, 183-194 (2015).

[214] Y. Murai, "Frictional drag reduction by bubble injection", Exp. Fluids 55, 1773 (2014).

[215] V. S. L'vov, A. Pomyalov, I. Procaccia, and V. Tiberkevich, "Drag reduction by microbubbles in turbulent flows: The limit of minute bubbles", Phys. Rev. Lett. 94, 174502 (2005).

[216] N. K. Madavan, S. Deutsch, and C. L. Merkle, "Measurements of local skin friction in a microbubble-modified turbulent boundary layer", J. Fluid Mech. 156, 237 (1985).

[217] S. L. Ceccio, "Friction drag reduction of external flows with bubble and gas injection", Annu. Rev. Fluid Mech. 42, 183-203 (2010).

[218] W. C. Sanders, E. S. Winkel, D. R. Dowling, M. Perlin, and S. L. Ceccio, "Bubble friction drag reduction in a high-reynolds-number flat-plate turbulent boundary layer", J. Fluid Mech. 552, 353-380 (2006).

[219] S. G. Huisman, D. P. M. van Gils, S. Grossmann, C. Sun, and D. Lohse, "Ultimate turbulent Taylor-Couette flow", Phys. Rev. Lett. 108, 024501 (2012).

[220] X. Zhu, R. A. Verschoof, D. Bakhuis, S. G. Huisman, R. Verzicco, C. Sun, and D. Lohse, "Wall roughness induces asymptotic ultimate turbulence.", Nat. Phys. 14, 417-423 (2018). 


\section{Summary}

Turbulent flows are omnipresent in nature and technology. The majority of flows encountered in daily life and in industrial applications deal with rough walls and transient effects. Furthermore, many flows can be regarded as multiphase flows, i.e. the flow consisting of multiple phases of liquids, gasses and solids. Surprisingly maybe, the understanding of these flows is still limited, and many studies focus on idealised situations, which do not take the aforementioned phenomena into account. To study these types of flow, we used a Taylor-Couette system, i.e. the flow between 2 concentric independently rotating cylinders. This system is one of the canonical flow setups in which the physics of fluids is studied, and it has been used to study a.o. pattern formation, instabilities, viscosity measurements, turbulence and multiphase flows. Taylor-Couette flow is known to be mathematically similar to Rayleigh-Bénard convection. That is, written in the correct dimensionless form, the relevant scaling laws are identical for both systems. In that sense, one can learn about RayleighBénard convection by studying Taylor-Couette flow, and vice versa.

In this thesis, we chose to specifically study transient effects, rough walls and air lubrication in turbulent flows, not only to increase our fundamental understanding of these of ubiquitous flows, but also to address highly relevant questions in collaboration with industrial partners. In maritime industry, the use of air lubrication is seen as a promising method to reduce the overall friction between a ship and the surrounding water, and thus the fuel consumption. However, the relevant parameters optimizing air lubrication are not yet well understood. Wall roughness is known to increase the drag, but given the enormous variety of roughness types, many open questions remain to be unanswered. The thesis is divided in three parts, i.e. Part 1: Transient turbulence, Part 2: Roughness in turbulence, and Part 3: Air lubrication in turbulent flows.

In part 1, we studied 2 different transient turbulent flows, i.e. decaying turbulence and periodically driven turbulence. We found that decaying turbulence cannot be described by a pure power law, contrasting the case of homogeneous and isotropic turbulence. In wall-bounded flow, the flow decays faster due to additional viscous drag applied by the walls. We found that the velocity profiles during the decay process are self-similar.

In chapter 2, we studied Taylor-Couette turbulence with periodically driven cylinders over a wide range of modulation periods, expressed here as the dimensionless Wom- 
ersley number, and compared our results to the numerically calculated laminar flow response. We find that, in contrast to the laminar case, the local flow response is independent of the distance to the modulated wall. Apparently, the turbulent mixing is sufficiently strong to prevent any radial dependence. However, the scalings of both the phase delay and amplitude response are similar to the laminar case.

In part 2, we studied the effect of riblets on both, or only one of the cylinders. We studied both global and local flow properties, making use of both numerical simulations and experiments. In chapter 3, we showed how wall roughness enhances the overall momentum transport. We found that when both walls are made rough, the boundary layers become independent of Reynolds number, and we reach the so-called 'asymptotic ultimate turbulent' regime, i.e. the upper limit of momentum transport. This regime was predicted by Kraichnan, Einstein's last assistant, more than fifty years ago. In chapter 4, we expand our analysis on these results by exploring the influence of riblet height on both local and global flow quantities.

In part 3, we focussed on various types of air lubrication. Firstly, we studied the mechanism behind bubble drag reduction. We added minute concentrations of the surfactant Triton X-100 into otherwise unchanged turbulent Taylor-Couette flow with bubbles. This resulted in reducing the drag reduction from over $40 \%$ to about $4 \%$, corresponding to the trivial effect of the reduced effective density. As the surfactant prevents bubble coalescence, and thus effectively makes the bubbles smaller, we conclude that bubble size and deformability are crucial for efficient bubble drag reduction.

Secondly, we studied so-called air cavities, which are air layers close to the wall, developed behind a rising edge, a so-called 'cavitator'. Air cavities are expected to effectively reduce the drag, as the wetted area of the solid wall is decreased significantly. We show that air cavities can be formed, and that size of the cavity increases with Reynolds number and void fraction, and that secondary flows destabilise the flow. Local air injection is not necessary, as long as air is present in the system it will be captured in the low-pressure wake of the cavitator. Although with air cavities we observe a gross drag reduction, overall we see a drag increase, as the added drag by the cavitators is larger than the drag reduction by the cavity.

Thirdly, we investigated how rough walls affect bubble drag reduction. We added rough ribs on both, either one, or none of the cylinders, and inject some global void fraction of air. We then measure the torque and the resulting drag reduction as compared to the case without bubbles. We here find two different regimes: (i) Strong drag reduction for the smooth-wall case and the case with ribs on the inner cylinder only. And (ii) weak drag reduction for the cases of ribs on the outer cylinder only and ribs on both cylinders. The underlying mechanism are turbulent Taylor vortices which are triggered when ribs are attached to the outer cylinder. Bubbles are trapped in these vortices, dragged away from the inner cylinder wall, and cannot decrease the drag effectively. In the case of ribs on the inner cylinder only, no Taylor rolls develop, and we still see remarkable drag reduction. We thus show that wall roughness can spoil air lubrication, if the roughness induces strong secondary flows. 


\section{Samenvatting}

Turbulente stromingen zijn overal aanwezig in de natuur en technische toepassingen. De meerderheid van de stromingen die we in het dagelijkse leven tegenkomen, ervaren oppervlakteruwheid en tijdsafhankelijke effecten. Daarnaast kunnen veel stromingen worden beschouwd als meerfase stromingen. Dat wil zeggen dat de stroming bestaat uit een mengsel van meerdere fases van vloeistoffen, gassen en solide deeltjes. Misschien verrassend, maar het fundamentele begrip van dit soort stromingen is nog steeds beperkt en veel wetenschappelijk werk onderzocht enkel geidealiseerde situaties, waarin de eerdergenoemde fenomenen geen rol spelen. Om deze stromingstypes wel goed te kunnen bestuderen hebben we 'Taylor-Couette stroming' gebruikt, d.w.z. de stroming tussen two concentrische, en onafhankelijk van elkaar roterende cylinders. Dit systeem is een van de typische testopstelling waarin vloeistoffysica wordt onderzocht. Het is gebruikt om bijvoorbeeld patroonvorming, instabiliteiten, viscositeitsmetingen, turbulentie en meerfase stromingen mee te onderzoeken. TaylorCouette stroming is wiskundig gezien te vergelijken met Rayleigh-Bénard convectie. Dat wil zeggen, wanneer de parameters in de correcte dimensieloze vorm zijn opgeschreven, zijn de relevante schaalwetten voor beide systemen gelijk. In die zin kunnen we iets over Rayleigh-Bénard convectie leren door Taylor-Couette stroming te bestuderen en vice versa. In dit proefschrift is ervoor gekozen om tijdsafhankelijke effecten, oppervlakteruwheid en luchtsmering in turbulente stromingen te bestuderen, met als doel om niet alleen het fundamentele begrip over deze overal aanwezige stromingen te vergroten, maar ook om een aantal belangrijke industriële vraagstukken te bestuderen. In de maritieme techniek wordt het gebruik van luchtsmering gezien als een veelbelovende techniek om de wrijving tussen een schip en het omringende water, en daarmee het brandstofverbruik te verminderen. Echter, de belangrijke parameters om luchtsmering te optimaliseren zijn nog niet goed begrepen. Oppervlakteruwheid staat erom bekend dat het de wrijving vergroot, maar gezien de enorme variëteit aan ruwheidstypes blijven vele vragen onbeantwoord. Dit proefschrift is verdeeld in 3 delen: Deel 1: Tijdsafhankelijke turbulentie, Deel 2: Ruwheid in turbulente stromingen, en Deel 3: Luchtsmering in turbulente stromingen.

In deel 1 hebben we twee verschillende tijdsafhankelijke stromingen onderzocht: het verval van turbulentie en periodiek aangedreven turbulentie. We vonden dat vervallende turbulentie niet kan worden beschreven door een pure machtsfunctie, in tegenstelling tot het verval van homogene en isotrope turbulentie. In deze wandbe- 
grenste stroming is het verval sneller door de toegevoegde viskeuze wrijving door de zijwanden. De genormaliseerde snelheidsprofielen blijven gelijk gedurende het vervalproces.

In hoofdstuk 2 hebben we Taylor-Couette turbulentie met periodiek aangedreven cylinders onderzocht. We varieerden de frequentie van de periode, hier uitgedrukt in het dimensieloze Womersley getal en vergeleken onze resultaten met de numeriek berekende respons van laminaire stroming. We vonden dat de lokale stromingsrespons, in tegenstelling tot de laminaire case, onafhankelijk is van de afstand tot de wand van de binnencylinder. Blijkbaar voorkomt de sterkte turbulente mixing elke radiale afhankelijkheid van de stromingsrespons. Echter, de schaalwetten van het faseverschil en de amplituderespons zijn vergelijkbaar voor de laminaire en turbulente gevallen.

In deel 2 hebben we gekeken naar het effect van transverse ribbels op beide of één van de cylinders. We hebben zowel lokale als globale stromingseigenschappen onderzocht, gebruik makend van zowel numerieke simulaties als experimenten. In hoofdstuk 3 lieten we zien hoe oppervlakteruwheid het globale impulstransport beïnvloedt. Als beide cylinders ruw zijn, wordt het gedrag in de grenslaag onafhankelijk van het Reynolds getal, en bereiken we het zogenaamde 'asymptotisch ultieme regime' van turbulentie, wat de opperste limiet is voor het impulstransport. Dit regime was door Robert Kraichnan, de laatste assistent van Albert Einstein, al meer dan 50 jaar geleden voorspeld. In hoofdstuk 4 breiden we onze analyse uit door de invloed van de ribhoogte te onderzoeken.

In deel 2 hebben we verschillende manieren van luchtsmering onderzocht. Ten eerste keken we naar het mechanisme achter luchtsmering met bellen. We voegden minitueuze concentraties toe van de oppervlakte-actieve stof Triton X-100 in TaylorCouette stroming met bubbels. Dit resulteerde in een verminderde wrijvingsreductie van meer dan $40 \%$ tot ongeveer $4 \%$, wat overeenkomt met het triviale effect van de verminderde effectieve dichtheid. De oppervlakte-actieve stof verhindert het clusteren van bubbels, waardoor de bubbels kleiner worden. Hieruit concluderen wij dat de bubbelgrootte en deformabiliteit cruciaal zijn voor effectieve wrijvingsreductie met bubbels.

Ten tweede keken we naar zogenoemde 'luchtholtes'. Dat zijn luchtlagen dicht bij de wand, die stabiel blijven met behulp van een 'cavitator' - een opstaande rand. Van luchtholtes wordt verwacht dat ze de wrijving efficient verminderen, aangezien deze het contactoppervlak tussen water en schip significant verminderen. We laten zien dat luchtholtes inderdaad kunnen ontstaan en dat hun grootte afhangt van Reynolds getal en de volumefractie lucht in de stroming. Secondaire stromingen destabiliseren de luchtholtes. Het lokaal injecteren van lucht is niet nodig; zolang voldoende lucht in het systeem aanwezig is kan deze lucht gevangen worden in het zog van de cavitator, waar immers de druk laag is. Hoewel we met luchtholtes aanzienlijke bruto wrijvingsreducties meten, neemt de netto wrijving neemt. De toegevoegde wrijving van de cavitators is groter dan de wrijvingsreductie door de luchtholtes.

Ten derde keken we naar hoe oppervlakteruwheid wrijvingsreductie met bellen beïnvloedt. We voegden transverse ribbels toe aan beide, één van beide of geen van de cylinders, en voegden een zekere hoeveelheid lucht toe aan het systeem. Vervolgens 
maten we de torsie en resulterende wrijvingsreductie, die we vergeleken met de case zonder bubbels. We vonden 2 regimes: (i) Sterke wrijvingsreductie voor de case zonder ribbels en de case met ribbels op de binnencylinder, en (ii) zwakke wrijvingsreductie voor de cases met ribbels op de buitencylinder en ribbels op beide cylinders.

Het onderliggende mechanisme wordt veroorzaakt door turbulente Taylor vortices, die getriggerd worden door de ribbels op de buitencylinder. In deze vortices worden bubbels gevangen en de bubbels worden weggezogen van de wand van de binnenste cylinder, waardoor ze de wrijving niet meer efficient beïnvloeden. In de met ribbels op alleen de binnenste cylinder ontstaan geen Taylor vortices en zien we dus nog steeds een opvallende wrijvingsreductie. Hiermee laten we zien dat, indien de ruwheid sterke secondaire stromingen triggert, luchtsmering nutteloos wordt. 


\section{Acknowledgements}

En zo zijn we aangekomen bij het enige hoofdstuk dat ook echt gelezen gaat worden door iedereen. De perfecte plek om wat mensen in het zonnetje te zetten. Ik ben immers ook veel dank verschuldigd aan vele mensen.

Dear Chao, you were able to enthuse me for this project within a few minutes after we first met. I really enjoyed being supervised by you. You're not only a knowledgable professor, but also a very nice guy to work with. This combination made you the best daily supervisor I could have imagined.

Dear Detlef, I enjoyed being part of your group during the last four years. I appreciate the way you lead our group: you don't have a need for control, but you give your students self-responsibility. By doing so, you stimulated me to make the most of myself. I thank you for your supervision and guidance these years, in which I learned tremendously.

Sander, eerst nog als mede-phd, uiteindelijk als supervisor. Ik dank je hartelijk voor de fijne manier waarop je me hebt ingewerkt in de materie, en nu betrokken bent bij het schrijven van de artikelen. Ik wens je al het beste in je verdere, ongetwijfeld succesvolle wetenschappelijke carrière.

Here, I would also like to thank the entire graduation committee: Kees Venner, Rob Lammertink, Tom van Terwisga and Gareth McKinley. Tom, jij gaf leiding aan het NWO-TTW project waar mijn werk, en dus deze thesis deel van uit maakt. Bedankt voor de manier waarop je dit project hebt geleid. Kees, bedankt voor jouw aandeel in mijn opleiding en de leuke samenwerking die we hadden met de supersone windtunnel. Rob and Gareth, many thanks for being part of my graduation committee.

The work presented in this thesis has been part of a NWO-TTW project, in which industrial partners and universities try to solve a certain problem or make progress in some field by closely collaborating together. I'd like to thank the program officers Ruben Scharpe, Xavier Weenink and Richard Nievaart for supervising this project on behalf of NWO-TTW. Furthermore, I'd like to thank Gem and Florian for our regular contact and stimulating meetings. Also of course our industrial partners from Damen Shipyards and MARIN, usually represented by Jochem the Jong, Oleksandr Zverkhovskyi and Evert-Jan Foeth.

Dennis, Rodrigo, Pim en Roeland. Vier volle jaren hebben we lief en leed in het lab gedeeld! Calibreren, balanceren, assembleren, meten, Futeks instellen tot zowel 
de Futeks als wijzelf volledig doordraaiden... De collegia sfeer in het lab was fijn, en we hebben er altijd weer wat moois van gemaakt. Een mooie mix van verschillende karakters met ieder weer z'n eigen expertise. Onder het genot van een bak koffie kwamen we altijd wel weer op een goed idee. Bedankt voor de samenwerking!

Arne, een klein jaar heb ik je mogen begeleiden. Je was een fijne student om mee te werken, en het begeleiden van jou heeft me echt over de befaamde "Phd motivatiedip" heen geholpen. Ik wens je al het beste!

Eerder in mijn opleiding waren het Harry Hoeijmakers en Niels Kruyt die mij enthousiast wisten te maken voor stromingsleer. Ik wil jullie beide hartelijk danken voor jullie rol in mijn opleiding en voor het begeleiden van mij gedurende mijn master.

Dan natuurlijk een dankwoord aan ons ondersteunend personeel: Bas, Martin, Joanita, Gert-Wim en Dennis. Wat moet de vakgroep zonder jullie? Gert-Wim, ik heb ervan genoten om samen met jou aan Solid-Works te zitten om zo telkens weer tot een goed ontwerp te komen. Ik heb veel van je geleerd! Bas, bedankt voor de talloze bestellingen, het fixen van mijn computer en het solderen van kabeltjes en stekkers. Martin, altijd ben ik weer onder de indruk van jouw expertise van de fijnmechanische constructietechnieken. Joanita, hoe het je lukt om altijd zo snel en goed van alles te regelen, ik weet het niet. In ieder geval, hartelijk dank hiervoor! Dennis, jij stond toch mooi aan de wieg van de Taylor-Couette opstelling, en nu ben je terug in de vakgroep. Fijn dat je altijd beschikbaar was voor vragen en discussie. Verder natuurlijk dank aan TCO, in het bijzonder aan Rindert en Geert voor jullie hulp aan onze opstelling. Antoinette, het kantoor was altijd weer prachtig schoon, en het was altijd gezellig om even een babbeltje te maken op de gang! Helemaal super!

I had great fun with many colleagues. Some if them I'll thank by name. I particularly enjoyed the crazy night in Boston (those present remember), the Denver mountain experience with Pieter, random talks with Pascal and Rianne, everyone who danced in the 'dome of sweat' at the FOM days, and course, my office mates Biljana, Arjan and Shantanu for just being there, and thanks for putting up with me.

The matches with PoF United were, beyond any doubt, among the weekly highlights. Having been the captain for several years, I saw our skill level improving by the match. Except for Ivans' of course. Enjoy, and keep up the good work! Een ander wekelijks hoogtepunt was het kibbeling eten met visclub Kapitein Iglo. Dank aan vishandel Rillmann voor vele familiezakken gebakken vis.

I'll leave the scientific world now. I thank all my (former) colleagues for four interesting years in this nuthouse for the well-educated. During my time as a PhD student I learned a lot, for which I owe much to many of you. However, not too deep down inside I am an engineer rather than a scientist, and I'm looking forward to a career at Demcon. I have had many discussions on the purpose and usefulness of doing science. To conclude on this issue I'd like to say the following. In the tradition of great scientists such as Rayleigh and Maxwell, I found the answer in Psalm 111:2, which reads: "Great are the works of the Lord, studied by all those who delight in them". And, I think I agree. The works of the Lord are definitely worthwhile to study.

In m'n ruim aanwezig vrije tijd heb ik me geen moment verveeld. Onder de streep, 
is werk natuurlijk een manier om je in leven te houden, en niet m'n 'core business' in het leven. Ik ben dan ook blij met alle vriendschap die ik de afgelopen jaren mocht ontvangen!

Om maar meteen met een mooie club vrienden te beginnen: de Schulti's! We hebben mooie dingen gedaan: kaarten met Tipsy Bridgie, hardlopend in de pijntjes polonaises, voetballen met ons team 1e FC Schultenbrau (met supportersvereniging 'Een blik waardig'), Pooltochten lopen, genieton met de mr. Visser Fahrradverein, hoekbanken, een klein straaljagertje op z'n tijd. Prachtige initiatieven, stuk voor stuk! De laatste jaren verandert de vriendschap: steeds vaker zijn alle vriendinnen erbij (helemaal prima), maar steeds minder vaak een mooi vuurtje (toch jammer). Eigenlijk kan ik me maar één ding wensen, dat is dat deze gekkigheid nog lang doorgaat.

Een speciaal woord van dank aan m'n trouwe klimmaten. Joris, al weer sinds 2006 zijn we tot nu toe elk jaar (!) samen op pad geweest. Van winnen op de Cengalo tot afzien in de kou in de Ecrins — we hebben alle stadia van hoogtepunten en moeheid wel meegemaakt samen! Tom, na mooie Dolomieten-avonturen nu meer samen aan het toerskieen. Samen met Arjen is dat ook een fantastisch avontuur. Een goede seilschaft is cruciaal, in het dagelijks leven zo ook als in de Alpen. En een goede seilschaft — dat zijn we! Bergheil op nog vele mooie avonturen!

Hartelijk dank aan m'n ouders, Mirjam \& Anton en David. David, nadat jij getuige was bij ons trouwen is de eervolle taak van paranimf nu Mirjam ten deel gevallen. Ik hoop dat ze het niet verprutst... Ik bedoel: je weet maar nooit! Zonder dollen: het is fijn te worden omringd door een lieve familie. En dat zijn jullie. Ik heb alweer veel zin in 2 mooie feesten binnenkort: de bruiloft van Mirjam \& Anton en de vakantie in Galtür.

Tot slot, de 2 belangrijkste dames in m'n leven. Anne, je hebt nog geen idee waar dit allemaal over gaat. Wees gerust, dat heeft bijna niemand. Ik vroeg mezelf ook vaak af wat ik aan het doen was. Je bent een prachtige lieve meid en ik hou van je. Jolanda, lieve Jolanda, wat zou ik toch zonder je moeten? De manier waarop je er altijd bent voor mij maakt dat ik elke dag opnieuw weer verliefd op je word. We zijn nog jong, en dat betekent dat we nog vele jaren van elkaar mogen houden! Daar heb ik heel veel zin in.

Tja, dat was 'm dan; volgens mij heb ik alles wel gezegd. Al het beste!

Ruben 


\section{About the author}

Ruben Adriaan Verschoof was born on October 7th, 1990 in Ede, the Netherlands. He graduated from high school 'CSG het Streek' in 2008, and started studying Mechanical Engineering at the University of Twente in Enschede. After a research internship in at GE Global Research in Munich, he graduated in 2014 on the aerodynamic design of fans under the supervision of prof. Harry Hoeijmakers and dr. Niels Kruyt. In the same year, he started his experimental $\mathrm{PhD}$ work in Applied Physics, in the Physics of Fluids group, supervised by prof. Chao Sun and prof. Detlef Lohse. His PhD work fo-

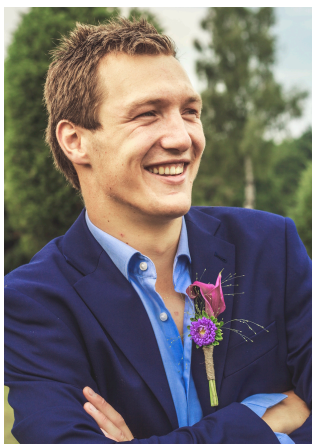
cussed on the understanding of air lubrication in highly turbulent flows, aiming to efficiently reduce the friction and fuel consumption in maritime industry. This study was performed in close collaboration with DAMEN Shipyards, the MARIN institute and the Delft University of Technology.

Apart from engineering, he takes in interest in alpine climbing, running, playing board- and card games, and socialising with friends and family. Ruben is married to Jolanda. Together they have one daughter, Anne. He enjoys family life every single day. 


\section{Publications}

- Bubble drag reduction requires large bubbles

R.A. Verschoof, R.C.A. van der Veen, C. Sun, D. Lohse

Phys. Rev. Lett., 117, 104502 (2016)

- Self-similar decay of high Reynolds number Taylor-Couette turbulence

R.A. Verschoof, S.G. Huisman, R.C.A. van der Veen, C. Sun, D. Lohse

Phys. Rev. Fluids, 1, 062402(R) (2016)

- Wall roughness induces asymptotic ultimate turbulence

X. Zhu ${ }^{\dagger}$, R.A. Verschoof ${ }^{\dagger}$, D. Bakhuis, S.G. Huisman, R. Verzicco, C. Sun, D. Lohse

Nat. Phys., 14, 417-423 (2018)

- Periodically driven Taylor-Couette turbulence

R.A. Verschoof ${ }^{\dagger}$, A. K. te Nijenhuis ${ }^{\dagger}$, S. G. Huisman, C. Sun, D. Lohse Accepted for publication at J. Fluid Mech (2018)

- Air cavities in turbulent Taylor-Couette flow

R.A. Verschoof, D. Bakhuis, P.A. Bullee, S. G. Huisman, C. Sun, D. Lohse Accepted for publication at Int. J. Multiphase Flow (2018)

- The influence of roughness on bubbly drag reduction in Taylor-Couette turbulence

R.A. Verschoof, D. Bakhuis, P.A. Bullee, S.G. Huisman, C. Sun, D. Lohse Under review

- Rough wall turbulent Taylor-Couette flow: the effect of the rib height

R.A. Verschoof, X. Zhu, D. Bakhuis, R. Verzicco, S.G. Huisman, C. Sun, D. Lohse

Under review

- Finite-sized rigid spheres in turbulent Taylor-Couette flow

D. Bakhuis, R.A. Verschoof, V. Mathai, S. G. Huisman, D. Lohse, C. Sun Under review

- The orientation of fibers in highly turbulent Taylor-Couette flow D. Bakhuis, R.A. Verschoof, S.G. Huisman, C. Sun, D. Lohse In preparation 
- Taylor-Couette turbulence with a superhydrophobic inner cylinder

P.A. Bullee, R.A. Verschoof, D. Bakhuis, R. Ezeta, S.G. Huisman, C. Sun, D. Lohse, R.G.H. Lammertink

In preparation 